\title{
QUANTUM SUPERGROUPS V. BRAID GROUP ACTION
}

\author{
SEAN CLARK AND DAVID HILL
}

\begin{abstract}
We construct a braid group action on quantum covering groups. We further use this action to construct a PBW basis for the positive half in finite type which is pairwise-orthogonal under the inner product. This braid group action is induced by operators on the integrable modules; however, these operators satisfy spin braid relations.
\end{abstract}

\section{INTRODUCTION}

The action of the Weyl group $W$ on the Cartan subalgebra of a Kac-Moody algebra $\mathfrak{g}$ can be lifted to an action of the braid group $B_{W}$ on the enveloping algebra of $\mathfrak{g}$ and its integrable representations. Lusztig [L, L2] generalized this construction to the quantum group $\mathbf{U}_{q}(\mathfrak{g})$ to give an action of $B_{W}$ on integrable representations of $U_{q}(\mathfrak{g})$ via certain operators defined on each weight space. Furthermore, these operators induce a compatible action of the braid group on the quantized enveloping algebra itself.

This action of $B_{W}$ has been used by Lusztig [L3 to construct a family of PBW bases for the half-quantum group when the associated Cartan datum is of finite type, one for each reduced expression of the longest word in $W$. This construction was generalized by Beck $[\mathrm{B}$ to produce a convex PBW basis in affine type. The action also has implications in the program of categorification, where a (strong) categorical action of $\mathfrak{g}$ induces a categorical action of $B_{W}$ on an associated category via auto-equivalences [CR, CKL, CK].

The papers [CHW1, CHW2, CFLW, C] introduced and studied the properties of quantum covering groups $\mathbf{U}=U_{q, \pi}(\mathfrak{g})$. These algebras allow for the study of both Drin'feld-Jimbo quantum groups of Kac-Moody Lie algebras alongside the quantum supergroup associated to anisotropic Kac-Moody Lie superalgebras via the new "half-parameter" $\pi$ (first introduced in [HW]), which satisfies $\pi^{2}=1$. Most of the structural features of quantum groups have incarnations in the quantum covering groups; for example, the quantum covering group admits a triangular decomposition and the Chevalley generators satisfy higher Serre relations. Additionally, the papers CHW2, CFLW] established the existence of a canonical basis for quantum covering groups which specializes to the Lusztig-Kashiwara canonical basis when $\pi=1$.

In this paper, we will construct a braid group action on the quantum covering group $\mathbf{U}$ using similar methods to [L, Part V]. In particular, we first define certain operators on integrable U-modules. These operators generalize Lusztig's construction, but come with additional factors of $\pi$ on each summand. The operators are constructed by quantum exponentials of Chevalley generators, and in general may not preserve the $\mathbb{Z} / 2 \mathbb{Z}$-grading of the modules. As a result, these operators do not necessarily satisfy braid relations; rather, they satisfy spin braid relations on

Date: January 21, 2020. 
isotypical components. In particular, though our approach to this construction largely mimics Lusztig's, it often requires subtle and nontrivial work to introduce the powers of $\pi$ in the various formulae. Nevertheless, most of Lusztig's results admit analogues: these operators induce even automorphisms of $\mathbf{U}$; the automorphisms preserve the integral form of $\mathbf{U}$; and they satisfy the braid relations. As a result, we can construct a family of orthogonal PBW-type bases for the quantum covering group associated to $\mathfrak{o s p}(1 \mid 2 n)$.

We note that in [CHW3, a family of PBW-type bases for $\mathbf{U}_{q}(\mathfrak{o s p}(1 \mid 2 n))$ have been constructed via the combinatorics of Lyndon words. We conjecture that these PBW bases should coincide with the PBW bases constructed via braid operators whenever the reduced expression for the longest word is induced from a total ordering on the simple roots. We also conjecture that PBW-type bases can be constructed in affine type using methods similar to those in [B].

The paper is organized as follows.

In Section 2, we set notations and recall some of the standard facts about quantum covering groups.

In Section 3, we introduce the braid group operators on integrable modules, and deduce some basic properties. These operators are used to construct automorphisms of $\mathbf{U}$. Additionally, the interaction between the braid operators and the coproduct are determined.

In Section 4, the braid automorphisms are considered as maps on subspaces of the positive half-quantum group. In particular, it is shown that the standard bilinear form is invariant under the braid operators up to a factor of an integral power of $\pi$.

In Section 5, we show that the braid automorphisms of $\mathbf{U}$ satisfy the braid relations, whereas the braid operators on integrable modules within certain blocks satisfy spin braid relations. In particular, the braid automorphisms are used to produce a PBW basis in finite type.

Acknowledgements. We would like to thank Weiqiang Wang for his interest in the paper and his helpful comments, as well as for the encouragement to complete this project.

\section{Preliminaries}

In this section, we recall notation and results on quantum covering groups from CHW1.

2.1. Root data. Let $I=I_{\overline{0}} \cup I_{\overline{1}}$ be a $\mathbb{Z}_{2}$-graded finite set of size $\ell$, for which we assume throughout that $I_{\overline{1}} \neq \emptyset$. Let $A=\left(a_{i j}\right)_{i, j \in I}$ be a generalized Cartan matrix (GCM) such that

(C1) $a_{i i}=2$, for all $i \in I$;

(C2) $a_{i j} \in \mathbb{Z}_{\leq 0}$, for $i \neq j \in I$;

(C3) $a_{i j}=0$ if and only if $a_{j i}=0$;

(C4) there exists an invertible matrix $D=\operatorname{diag}\left(d_{1}, \ldots, d_{r}\right)$ with $D A$ symmetric.

We can and shall further assume $d_{i} \in \mathbb{Z}_{>0}$ and $\operatorname{gcd}\left(d_{1}, \ldots, d_{r}\right)=1$. We also define the symbols $b_{i j}=1-a_{i j}$.

Introduce the parity function $p(i)=0$ for $i \in I_{\overline{0}}$ and $p(i)=1$ for $i \in I_{\overline{1}}$. Throughout the paper, we will impose the additional assumption: 
(P1) $a_{i j} \in 2 \mathbb{Z}$, for all $i \in I_{\overline{1}}$ and all $j \in I$;

(P2) for all $i \in I, d_{i} \equiv p(i)(\bmod 2)$.

We note that (P2) is almost always satisfied for Cartan data of finite or affine type satisfying $(\mathrm{P} 1)$.

Let $\left(P, P^{\vee}, \Pi, \Pi^{\vee}\right)$ be the root data associated to $A$. Here, $P$ and $P^{\vee}$ are free $\mathbb{Z}$-modules of rank $\ell$ (called the weight and coweight lattice, respectively). The simple roots (resp. coroots)

$$
\Pi=\left\{\alpha_{i} \mid i \in I\right\} \subset P \quad\left(\text { resp. } \Pi^{\vee}=\left\{\alpha_{i}^{\vee} \mid i \in I\right\} \subset P^{\vee}\right)
$$

are linearly independent, and we define the root lattice

$$
Q=\sum_{i \in I} \mathbb{Z} \alpha_{i} \text { and } Q_{+}=\sum_{i \in I} \mathbb{Z}_{\geq 0} \alpha_{i} .
$$

Furthermore, for $\nu=\sum \nu_{i} \alpha_{i}^{\vee}$ with $\nu_{i} \in \mathbb{Z}$, we define the notation

$$
\tilde{\nu}=\sum d_{i} \nu_{i} \alpha_{i}^{\vee} \text {. }
$$

We may define a $\mathbb{Z}_{2}$-grading on $Q$ by declaring $p\left(\alpha_{i}\right)=p(i)$ and extending linearly. We also have a $\mathbb{Z}$-grading on $Q$ given by ht $\left(\sum_{i \in I} c_{i} \alpha_{i}\right)=\sum_{i \in I} c_{i}$.

Let

$$
\langle\cdot, \cdot\rangle: P^{\vee} \times P \longrightarrow \mathbb{Z}
$$

denote the perfect pairing defined by $\left\langle\alpha_{i}^{\vee}, \alpha_{j}\right\rangle=a_{i j}$, and let $\omega_{i} \in P\left(\right.$ resp. $\left.\omega_{i}^{\vee} \subset P^{\vee}\right)$ be dual to $\alpha_{i}^{\vee}$ (resp. $\alpha_{i}$ ) with respect to this pairing. We set $P_{+}=\left\{\lambda \in P \mid\left\langle\alpha_{i}^{\vee}, \lambda\right\rangle \geq 0\right\}$.

Also, define the symmetric bilinear form

$$
(\cdot, \cdot): Q \times Q \longrightarrow \mathbb{Z}
$$

by $\left(\alpha_{i}, \alpha_{j}\right)=d_{i} a_{i j}$. Observe that conditions (P1) and (P2) together imply that $(\mu, \nu) \in 2 \mathbb{Z}$ for any $\mu, \nu \in Q$, hence in particular $\langle\tilde{\mu}, \nu\rangle \in 2 \mathbb{Z}$ for any $\mu \in Q^{\vee}$ and $\nu \in Q$.

2.2. The braid group and spin braid group. The braid group $B=B(A)$ associated to a GCM $A$ is defined to be the group with generators $t_{i}(i \in I)$ subject to the relations

$$
\underbrace{t_{i} t_{j} t_{i} \cdots}_{m_{i j}}=\underbrace{t_{j} t_{i} t_{j} \cdots}_{m_{i j}}
$$

where the number of terms, $m_{i j}$, is determined by the product $a_{i j} a_{j i}$ as follows:

$$
\begin{array}{c|ccccc}
a_{i j} a_{j i} & 0 & 1 & 2 & 3 & \geq 4 \\
\hline m_{i j} & 2 & 3 & 4 & 6 & \infty
\end{array}
$$

The braid group acts on $P$ and $P^{\vee}$ via simple reflections. To wit, for $i \in I$, we define the simple reflection $s_{i}$, which acts on $P$ (resp. $P^{\vee}$ ) by the formula

$$
s_{i}(\lambda)=\lambda-\left\langle\alpha_{i}^{\vee}, \lambda\right\rangle \alpha_{i}, \quad\left(\text { resp. } s_{i}\left(\lambda^{\vee}\right)=\lambda^{\vee}-\left\langle\lambda^{\vee}, \alpha_{i}\right\rangle \alpha_{i}^{\vee}\right) .
$$

The Weyl group $W$ is the group generated by the set of reflections $\left\{s_{i} \mid i \in I\right\}$. It is subject to the relations $s_{i}^{2}=1$ for $i \in I$ and the braid relations (2.2) (with $t_{i}, t_{j}$ replaced by $s_{i}, s_{j}$ ).

In addition to these standard definitions, we shall need a variant of the braid group. We define the spin braid group $B^{\text {spin }}=B^{\text {spin }}(A, \varpi)$ associated to a GCM $A$ and parity function $\varpi: I \rightarrow\{0,1\}$ as follows. Define the set of $I_{\text {spin }} \subset I \times I$ via $I_{\text {spin }}=\left\{(i, j) \in I \times I \mid \varpi(i)=\varpi(j)=1\right.$, and $\left.a_{i j}=0\right\}$. Then, $B^{\text {spin }}$ is the group 
with generators $t_{i}(i \in I)$ and an additional generator $\varsigma$ satisfying the following relations:

(SB1) $\varsigma^{2}=1$ and $\varsigma t_{i}=t_{i} \varsigma$ for all $i \in I$;

(SB2) if $(i, j) \notin I_{\text {spin }}, t_{i}$ and $t_{j}$ satisfy (2.2);

(SB3) if $(i, j) \in I_{\text {spin }}$, then $t_{i} t_{j}=\varsigma t_{j} t_{i}$.

2.3. Parameters. Let $q$ be a formal parameter and let $\pi$ be an indeterminate such that

$$
\pi^{2}=1
$$

For a ring $R$, we define $R^{\pi}=R[\pi] /\left(\pi^{2}-1\right)$. We will work over (subrings of) the ring $\mathbb{Q}^{\pi}(q)$. This ring has idempotents

$$
\varepsilon_{+}=\frac{1+\pi}{2}, \quad \varepsilon_{-}=\frac{1-\pi}{2},
$$

and note that $\mathbb{Q}^{\pi}(q)=\mathbb{Q}(q) \varepsilon_{+} \oplus \mathbb{Q}(q) \varepsilon_{-}$. In particular, since $\pi \varepsilon_{ \pm}= \pm \varepsilon_{ \pm}$for any $\mathbb{Q}^{\pi}(q)$-module $M$, we see that

$$
\left.M\right|_{\pi= \pm 1} \cong \varepsilon_{ \pm} M \text {. }
$$

Let $\mathcal{A}=\mathbb{Z}^{\pi}\left[q, q^{-1}\right]$. For $k \in \mathbb{Z}_{\geq 0}$ and $n \in \mathbb{Z}$, we use a $(q, \pi)$-variant of quantum integers, quantum factorial and quantum binomial coefficients:

$$
\begin{aligned}
{[n]_{q, \pi} } & =\frac{(\pi q)^{n}-q^{-n}}{\pi q-q^{-1}} \in \mathcal{A}, \\
{[n]_{q, \pi}^{!} } & =\prod_{l=1}^{n}[l]_{q, \pi} \in \mathcal{A}, \\
{\left[\begin{array}{l}
n \\
k
\end{array}\right]_{q, \pi} } & =\frac{\prod_{l=n-k+1}^{n}\left((\pi q)^{l}-q^{-l}\right)}{\prod_{m=1}^{k}\left((\pi q)^{m}-q^{-m}\right)} \in \mathcal{A} .
\end{aligned}
$$

These $(q, \pi)$-quantum integers satisfy identities analogous to more traditional quantum integers.

$$
\begin{aligned}
& {\left[\begin{array}{l}
a \\
t
\end{array}\right]_{q, \pi}=(-1)^{t} \pi^{t a-\left(\begin{array}{c}
t \\
2
\end{array}\right)}\left[\begin{array}{c}
t-a-1 \\
t
\end{array}\right]_{q, \pi},} \\
& {\left[\begin{array}{l}
a \\
t
\end{array}\right]_{q, \pi}=\left\{\begin{array}{ll}
\frac{[a]_{q, \pi}^{!}}{[t]_{q, \pi}^{!}[a-t]_{q, \pi}^{!}} & \text {if } 0 \leq t \leq a \\
0 & \text { if } a<t
\end{array} \quad \text { if } a \geq 0,\right.}
\end{aligned}
$$

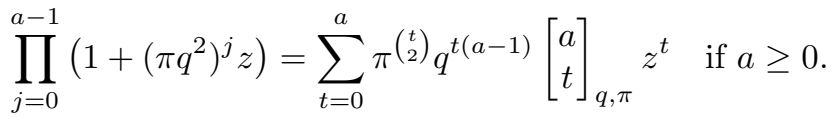

Here $z$ is another indeterminate. If $a^{\prime}, a^{\prime \prime}$ are integers and $t \in \mathbb{N}$, then

$$
\left[\begin{array}{c}
a^{\prime}+a^{\prime \prime} \\
t
\end{array}\right]_{q, \pi}=\sum_{t^{\prime}+t^{\prime \prime}=t} \pi^{t^{\prime} t^{\prime \prime}+a^{\prime} t^{\prime \prime}} q^{a^{\prime} t^{\prime \prime}-a^{\prime \prime} t^{\prime}}\left[\begin{array}{l}
a^{\prime} \\
t^{\prime}
\end{array}\right]_{q, \pi}\left[\begin{array}{l}
a^{\prime \prime} \\
t^{\prime \prime}
\end{array}\right]_{q, \pi} .
$$

We note the following specializations of some of the above identities. Observe that

$$
\left[\begin{array}{c}
-1 \\
t
\end{array}\right]_{q, \pi}=(-1)^{t} \pi^{\left(\begin{array}{c}
t+1 \\
2
\end{array}\right)}
$$


for any $t \geq 0, i \in I$. Furthermore if $a \geq 1$, then we have

$$
\sum_{t=0}^{a}(-1)^{t} \pi^{\left(\begin{array}{c}
t \\
2
\end{array}\right)} q^{t(a-1)}\left[\begin{array}{l}
a \\
t
\end{array}\right]_{q, \pi}=0
$$

which follows from (2.7) by setting $z=-1$.

We will use the notation

$$
q_{i}=q^{d_{i}}, \quad \pi_{i}=\pi^{d_{i}}, \quad \text { for } i \in I .
$$

More generally, for $\nu=\sum \nu_{i} \alpha_{i}$, we set

$$
q_{\nu}=\prod_{i \in I} q_{i}^{\nu_{i}}, \quad \pi_{\nu}=\prod_{i \in I} \pi_{i}^{\nu_{i}}
$$

We also extend this notation to quantum integers, factorials, and binomial coefficients; that is, we set

$$
[n]_{i}=[n]_{q_{i}, \pi_{i}}, \quad[n]_{i}^{!}=[n]_{q_{i}, \pi_{i}}^{!}, \quad\left[\begin{array}{c}
n \\
k
\end{array}\right]_{i}=\left[\begin{array}{l}
n \\
k
\end{array}\right]_{q_{i}, \pi_{i}} .
$$

The bar involution on $\mathbb{Q}^{\pi}(q)$ is the $\mathbb{Q}^{\pi}$-algebra automorphism defined by $\overline{f(q)}=$ $f\left(\pi q^{-1}\right)$ for $f(q) \in \mathbb{Q}^{\pi}(q)$. We note that the bar involution restricts to a $\mathbb{Z}^{\pi}$-algebra automorphism of $\mathcal{A}$ and that the $(q, \pi)$-integers are bar-invariant.

2.4. The quantum covering groups. We recall some definitions from [CHW1].

Definition 2.4.1. CHW1 The half-quantum covering group $\mathbf{f}$ associated to the anisotropic datum $(I, \cdot)$ is the $Q^{+}$-graded $\mathbb{Q}^{\pi}(q)$-algebra on the generators $\theta_{i}$ for $i \in I$ with $\left|\theta_{i}\right|=\alpha_{i}$, satisfying the relations

$$
\sum_{k=0}^{b_{i j}}(-1)^{k} \pi^{\left(\begin{array}{c}
k \\
2
\end{array}\right) p(i)+k p(i) p(j)}\left[\begin{array}{c}
b_{i j} \\
k
\end{array}\right]_{i} \theta_{i}^{b_{i j}-k} \theta_{j} \theta_{i}^{k}=0(i \neq j),
$$

where here recall that $b_{i j}=1-a_{i j}$ (cf. §2.1).

We define the divided powers

$$
\theta_{i}^{(n)}=\theta_{i}^{n} /[n]_{i}^{!}
$$

Let ${ }_{\mathcal{A}} \mathbf{f}$ be the $\mathcal{A}$-algebra generated by $\theta_{i}^{(n)}$ for various $i \in I, n \in \mathbb{N}$.

The algebra $\mathbf{f}$ admits a coproduct structure. To wit, we equip $\mathbf{f} \otimes \mathbf{f}$ with the twisted multiplication

$$
(x \otimes y)\left(x^{\prime} \otimes y^{\prime}\right)=\pi^{p\left(x^{\prime}\right) p(y)} q^{-\left(\left|x^{\prime}\right|,|y|\right)}\left(x x^{\prime}\right) \otimes\left(y y^{\prime}\right),
$$

and obtain a $\mathbb{Q}^{\pi}(q)$-algebra homomorphism $r: \mathbf{f} \rightarrow \mathbf{f} \otimes \mathbf{f}$ satisfying $r\left(\theta_{i}\right)=\theta_{i} \otimes 1+$ $1 \otimes \theta_{i}$. We note that this map satisfies

$$
r\left(\theta_{i}^{(n)}\right)=\sum_{s+t=n}(\pi q)^{-s t} \theta_{i}^{(s)} \otimes \theta_{i}^{(t)}
$$

There are unique $\mathbb{Q}^{\pi}(q)$-linear maps $r_{i},{ }_{i} r: \mathbf{f} \rightarrow \mathbf{f}$ for each $i \in I$ such that $r_{i}(1)=$ ${ }_{i} r(1)=0$ and $r_{i}\left(\theta_{j}\right)={ }_{i} r\left(\theta_{j}\right)=\delta_{i j}$ and satisfying

$$
\begin{aligned}
& { }_{i} r(x y)={ }_{i} r(x) y+\pi^{p(x) p(i)} q^{-\left(|x|, \alpha_{i}\right)} x_{i} r(y), \\
& r_{i}(x y)=\pi^{p(y) p(i)} q^{-\left(|y|, \alpha_{i}\right)} r_{i}(x) y+x r_{i}(y) .
\end{aligned}
$$

Moreover, $r(x)=r_{i}(x) \otimes \theta_{i}+\theta_{i} \otimes{ }_{i} r(x)+$ (other bi-homogeneous terms). 
Finally, we recall that $\mathbf{f}$ comes equipped with a symmetric bilinear form $(-,-)$ satisfying

$$
\begin{aligned}
(1,1) & =1 ; \\
\left(\theta_{i}, \theta_{j}\right) & =\delta_{i j}\left(1-\pi_{i} q_{i}^{2}\right)^{-1} \quad(\forall i, j \in I) ; \\
\left(x, y^{\prime} y^{\prime \prime}\right) & =\left(r(x), y^{\prime} \otimes y^{\prime \prime}\right) \quad\left(\forall x, y^{\prime}, y^{\prime \prime} \in \mathbf{f} .\right.
\end{aligned}
$$

Here, the induced bilinear form $\mathbf{f} \otimes \mathbf{f}$ on $\mathbf{f}$ is given by

$$
\left(x_{1} \otimes x_{2}, x_{1}^{\prime} \otimes x_{2}^{\prime}\right):=\left(x_{1}, x_{1}^{\prime}\right)\left(x_{2}, x_{2}^{\prime}\right),
$$

for homogeneous $x_{1}, x_{2}, x_{1}^{\prime}, x_{2}^{\prime} \in \mathbf{f}$. In particular, for all $x, y \in \mathbf{f}$ we have

$$
\left(\theta_{i} x, y\right)=\left(\theta_{i}, \theta_{i}\right)\left(x,{ }_{i} r(y)\right), \quad\left(x \theta_{i}, y\right)=\left(\theta_{i}, \theta_{i}\right)\left(x, r_{i}(y)\right) .
$$

Definition 2.4.2. CHW1 The quantum covering group $\mathbf{U}$ associated to the datum $\left(P, P^{\vee}, \Pi, \Pi^{\vee}\right)$ is the $\mathbb{Q}^{\pi}(q)$-algebra with generators $E_{i}, F_{i}, K_{\mu}$, and $J_{\mu}$, for $i \in I$ and $\mu \in P^{\vee}$, subject to the relations:

$$
\begin{gathered}
J_{\mu} J_{\nu}=J_{\mu+\nu}, \quad K_{\mu} K_{\nu}=K_{\mu+\nu}, \quad K_{0}=J_{0}=J_{\nu}^{2}=1, \quad J_{\mu} K_{\nu}=K_{\nu} J_{\mu}, \\
J_{\mu} E_{i}=\pi^{\left\langle\mu, \alpha_{i}\right\rangle} E_{i} J_{\mu}, \quad J_{\mu} F_{i}=\pi^{-\left\langle\mu, \alpha_{i}\right\rangle} F_{i} J_{\mu}, \\
K_{\mu} E_{i}=q^{\left\langle\mu, \alpha_{i}\right\rangle} E_{i} K_{\mu}, \quad K_{\mu} F_{i}=q^{-\left\langle\mu, \alpha_{i}\right\rangle} F_{i} K_{\mu}, \\
E_{i} F_{j}-\pi^{p(i) p(j)} F_{j} E_{i}=\delta_{i j} \frac{J_{d_{i} i} K_{d_{i} \alpha_{i}^{\vee}}-K_{-d_{i} \alpha_{i}^{\vee}}}{\pi_{i} q_{i}-q_{i}^{-1}} \\
\sum_{k=0}^{b_{i j}}(-1)^{k} \pi^{\left(\begin{array}{c}
k \\
2
\end{array}\right) p(i)+k p(i) p(j)}\left[\begin{array}{c}
b_{i j} \\
k
\end{array}\right]_{i} E_{i}^{b_{i j}-k} E_{j} E_{i}^{k}=0 \quad(i \neq j), \\
\sum_{k=0}^{b_{i j}}(-1)^{k} \pi^{\left(\begin{array}{c}
k \\
2
\end{array}\right) p(i)+k p(i) p(j)}\left[\begin{array}{c}
b_{i j} \\
k
\end{array}\right]_{i} F_{i}^{b_{i j}-k} F_{j} F_{i}^{k}=0(i \neq j),
\end{gathered}
$$

for $i, j \in I$ and $\mu, \nu \in P^{\vee}$.

We endow $\mathbf{U}$ with a $Q_{+}$-grading by setting

$$
\left|E_{i}\right|=\alpha_{i}, \quad\left|F_{i}\right|=-\alpha_{i}, \quad\left|J_{\mu}\right|=\left|K_{\mu}\right|=0,
$$

and also endow $\mathbf{U}$ with a $\mathbb{Z}_{2}$-grading by setting

$$
p\left(E_{i}\right)=p\left(F_{i}\right)=p(i), \quad p\left(J_{\mu}\right)=p\left(K_{\mu}\right)=0 .
$$

We set $\mathbf{U}_{\nu}=\{x \in \mathbf{U}:|x|=\nu\}$. Note that $p(x)=p(\nu)$ for all $x \in \mathbf{U}_{\nu}$. Henceforth, any equation involving $|-|$ or $p(-)$ implicitly assumes all the elements are homogeneous.

Let $\mathbf{U}^{+}$be the subalgebra generated by $E_{i}$ with $i \in I$, and $\mathbf{U}^{0}$ be the subalgebra generated by $K_{\nu}$ and $J_{\nu}$ for $\nu \in Y$. There is an isomorphisms $\mathbf{f} \rightarrow \mathbf{U}^{-}$(resp. $\mathbf{f} \rightarrow \mathbf{U}^{+}$) defined by $\theta_{i} \mapsto \theta_{i}^{-}=F_{i}$ (resp. $\theta_{i} \mapsto \theta_{i}^{+}=E_{i}$ ). The following proposition was proven in [CHW1].

Proposition 2.4.3. There is a triangular decomposition

$$
\mathbf{U} \cong \mathbf{U}^{-} \otimes \mathbf{U}^{0} \otimes \mathbf{U}^{+} \cong \mathbf{U}^{+} \otimes \mathbf{U}^{0} \otimes \mathbf{U}^{-} .
$$


We define the divided powers

$$
E_{i}^{(n)}=\left(\theta_{i}^{(n)}\right)^{+}, \quad F_{i}^{(n)}=\left(\theta_{i}^{(n)}\right)^{-},
$$

and set ${ }_{\mathcal{A}} \mathbf{U}^{ \pm}=\left({ }_{\mathcal{A}} \mathbf{f}\right)^{ \pm}$. We will also use the shorthand notations

$$
\tilde{J}_{i}=J_{d_{i} \alpha_{i}^{\vee}}, \quad \tilde{J}_{\nu}=J_{\tilde{\nu}}, \quad \tilde{K}_{i}=K_{d_{i} \alpha_{i}^{\vee}}, \quad \tilde{K}_{\nu}=K_{\tilde{\nu}} .
$$

Then for $\nu \in P^{\vee}$, we also have the $\nu$-integers and $\nu$-binomial coefficients

$$
[\nu ; n]=\frac{\pi_{\nu}^{n} v_{\nu}^{n} \tilde{J}_{\nu} \tilde{K}_{\nu}-\tilde{K}_{\nu}^{-1} v_{\nu}^{-n}}{\pi_{\nu} v_{\nu}-v_{\nu}^{-1}}, \quad\left[\begin{array}{c}
\nu ; n \\
k
\end{array}\right]=\frac{\prod_{s=1}^{k}[\nu ; n+1-k]}{[k]_{v_{\nu}, \pi_{\nu}}^{!}} .
$$

We let ${ }_{\mathcal{A}} \mathbf{U}$ be the $\mathcal{A}$-subalgebra of $\mathbf{U}$ generated by $E_{i}^{(n)}, F_{i}^{(n)}, J_{\nu}$, and $K_{\nu}$ for $i \in I$, $\nu \in Y, n \geq a \in \mathbb{N}$.

We have the following general commutation lemma. (See CHW1, Proposition 2.2.2].)

Proposition 2.4.4. For $x \in \mathbf{f}$ and $i \in I$, we have (in $\mathbf{U}$ )

(a) $\left[x^{+}, F_{i}\right]=\frac{\pi_{i}^{p(x)-p(i)} \tilde{J}_{i} \tilde{K}_{i}{ }_{i} r(x)^{+}-r_{i}(x)^{+} \tilde{K}_{-i}}{\pi_{i} q_{i}-q_{i}^{-1}}$,

(b) $\left[E_{i}, x^{-}\right]=\frac{\pi_{i}^{p(x)-p(i)} r_{i}(x)^{-} \tilde{J}_{i} \tilde{K}_{i}-\tilde{K}_{-i}{ }_{i} r(x)^{-}}{\pi_{i} q_{i}-q_{i}^{-1}}$.

Specializing this identity yields the following relation in ${ }_{\mathcal{A}} \mathbf{U}$.

Lemma 2.4.5. [CW, Lemma 2.8] For $i \in I$, and $N, M \geq 1$,

(a) $E_{i}^{(N)} F_{i}^{(M)}=\sum_{t \geq 0} \pi_{i}^{M N-\left({ }^{(+1}\right)} F_{i}^{(M-t)}\left[\begin{array}{c}\alpha_{i}^{\vee} ; 2 t-N-M \\ t\end{array}\right] E_{i}^{(N-t)}$,

(b) $F_{i}^{(N)} E_{i}^{(M)}=\sum_{t \geq 0}(-1)^{t} \pi_{i}^{M N-t(M+N)} E_{i}^{(M-t)}\left[\begin{array}{c}\alpha_{i}^{\vee} ; M+N-t-1 \\ t\end{array}\right] F_{i}^{(N-t)}$,

where we interpret $F_{i}^{(0)}=E_{i}^{(0)}=1$, and $F_{i}^{(s)}=E_{i}^{(s)}=0$ if $s<0$.

The algebra $\mathbf{U}$ has a number of important automorphisms, which we will now recall. There is a $\mathbb{Q}^{\pi}(q)$-algebra automorphism $\omega: \mathbf{U} \rightarrow \mathbf{U}$ defined by

$$
\omega\left(E_{i}\right)=\pi_{i} \tilde{J}_{i} F_{i}, \quad \omega\left(F_{i}\right)=E_{i}, \quad \omega\left(K_{\nu}\right)=K_{-\nu}, \quad \omega\left(J_{\nu}\right)=J_{\nu} .
$$

There is also an important anti-automorphism of $\mathbf{U}$. To wit, there is a $\mathbb{Q}^{\pi}(q)$ linear map $\sigma: \mathbf{U} \rightarrow \mathbf{U}$ such that

$$
\sigma\left(E_{i}\right)=E_{i}, \quad \sigma\left(F_{i}\right)=\pi_{i} \tilde{J}_{i} F_{i}, \quad \sigma\left(K_{\nu}\right)=K_{-\nu}, \quad \sigma\left(J_{\nu}\right)=J_{\nu},
$$

and satisfying

$$
\sigma(x y)=\sigma(y) \sigma(x) .
$$

The bar-involution on $\mathbf{U}$ is the $\mathbb{Q}^{\pi}$-algebra automorphism defined by

$$
\bar{E}_{i}=E_{i}, \quad \bar{F}_{i}=F_{i}, \quad \bar{K}_{\nu}=J_{\nu} K_{-\nu}, \quad \bar{J}_{\nu}=J_{\nu}, \quad \bar{q}=\pi q^{-1} .
$$

The maps $\omega, \sigma$, and - (or variations thereof) were defined in [CHW1]. 
Finally, we recall that $\mathbf{U}$ has a braided Hopf algebra structure. Specifically, endowing $\mathbf{U} \otimes \mathbf{U}$ with the multiplication $(x \otimes y)\left(x^{\prime} \otimes y^{\prime}\right)=\pi^{p\left(x^{\prime}\right) p(y)}\left(x x^{\prime}\right) \otimes\left(y y^{\prime}\right)$, the map $\Delta: \mathbf{U} \rightarrow \mathbf{U} \otimes \mathbf{U}$ satisfying

$$
\begin{aligned}
\Delta\left(E_{i}\right) & =E_{i} \otimes 1+\tilde{J}_{i} \tilde{K}_{i} \otimes E_{i} \quad(i \in I) \\
\Delta\left(F_{i}\right) & =F_{i} \otimes \tilde{K}_{i}^{-1}+1 \otimes F_{i} \quad(i \in I) \\
\Delta\left(K_{\mu}\right) & =K_{\mu} \otimes K_{\mu} \quad(\mu \in Y) \\
\Delta\left(J_{\mu}\right) & =J_{\mu} \otimes J_{\mu} \quad(\mu \in Y) .
\end{aligned}
$$

is an algebra homomorphism. This is related to the coproduct $r$ on $\mathbf{f}$ as follows. Given $x \in \mathbf{f}$ such that $r(x)=\sum x_{1} \otimes x_{2}$, then

$$
\begin{aligned}
& \Delta\left(x^{+}\right)=\sum \pi^{p\left(x_{1}\right) p\left(x_{2}\right)} q^{\left(\left|x_{1}\right|,\left|x_{2}\right|\right)} x_{2}^{+} \tilde{J}_{\left|x_{2}\right|} \tilde{K}_{\left|x_{2}\right|} \otimes x_{1}^{+} \\
& \Delta\left(x^{-}\right)=\sum x_{1}^{-} \otimes \tilde{K}_{-\left|x_{1}\right|} x_{2}^{-}
\end{aligned}
$$

Moreover, we have the formulas

$$
\begin{aligned}
& \Delta\left(E_{i}^{(p)}\right)=\sum_{p^{\prime}+p^{\prime \prime}=p} q_{i}^{p^{\prime} p^{\prime \prime}} \tilde{J}_{i}^{p^{\prime \prime}} E_{i}^{\left(p^{\prime}\right)} \tilde{K}_{i}^{p^{\prime \prime}} \otimes E_{i}^{\left(p^{\prime \prime}\right)}, \\
& \Delta\left(F_{i}^{(p)}\right)=\sum_{p^{\prime}+p^{\prime \prime}=p}\left(\pi_{i} q_{i}\right)^{-p^{\prime} p^{\prime \prime}} F_{i}^{\left(p^{\prime}\right)} \otimes \tilde{K}_{i}^{-p^{\prime}} F_{i}^{\left(p^{\prime \prime}\right)} .
\end{aligned}
$$

2.5. Representation categories. In this paper, a $\mathbf{U}$-module will always mean a $\mathbb{Q}^{\pi}(q)$-module which carries a $\mathbf{U}$-action and a $\mathbb{Z} / 2 \mathbb{Z}$-grading compatible with the action. Recall that a weight module for $\mathbf{U}$ is a $\mathbf{U}$-module $M$ such that

$$
M=\bigoplus_{\lambda \in P} M_{\lambda}, \quad M_{\lambda}=\left\{m \in M \mid K_{\mu} m=q^{\langle\mu, \lambda\rangle} m \text { for any } \mu \in P^{\vee}\right\} .
$$

We say that a weight module $M$ is $\pi$-free if $M_{\lambda}$ is free as a $\mathbb{Q}^{\pi}(q)$-module. Henceforth, we shall always assume a $\mathbf{U}$-module is a $\pi$-free weight module.

An important subcategory of $\mathbf{U}$-modules is the category $\mathcal{O}$ of $\pi$-free weight modules $M$ such that for any $m \in M$, there exists an $N$ such that $x^{+} m=0$ for any $x \in \mathbf{f}$ with ht $|x|>N$. The category $\mathcal{O}$ in turn has an important subcategory $\mathcal{O}_{\text {int }}$ formed by its integrable modules; that is, modules $M \in \mathcal{O}$ such that $E_{i}$ and $F_{i}$ act locally nilpotently for all $i \in I$. We recall from [CHW1, §2.6] that $\mathcal{O}_{\text {int }}$ is completely reducible, with simple modules $V(\lambda)$ for $\lambda \in P_{+}$. Moreover, these modules arise as quotients of standard highest weight modules $M(\lambda)$ (each of which is isomorphic to $\mathbf{f}$ as a vector space).

When studying the braid group action, it is often sufficient to restrict attention to a particular simple root. To that end, let $\mathbf{U}(i)$ be the subalgebra of $\mathbf{U}$ generated by $E_{i}, F_{i}, \tilde{K}_{i}$ and $\tilde{J}_{i}$. We define the notation $\mathcal{O}^{i}$ (respectively, $\mathcal{O}_{\text {int }}^{i}$ ) for the corresponding categories of $\mathbf{U}(i)$-modules. Then the weights of $\mathbf{U}(i)$ may, and shall, be identified with integers $\mathbb{Z}$ (see $[\mathrm{CW}]$ ).

From Lemma 2.4.5 we have the following immediate corollary.

Corollary 2.5.1. Let $M \in \mathcal{O}_{\text {int }}^{i}$, and let $m \in \mathbb{Z}_{\geq 0}$. Assume $\eta \in M_{m}$ satisfies $E_{i} \eta=0$, and let $\xi=F_{i}^{(m)} \eta$. Then, for $k, h \geq 0$ such that $k+h=m$,

$$
F_{i}^{(k)} \eta=\pi_{i}^{m h+\left(\begin{array}{c}
h+1 \\
2
\end{array}\right)} E_{i}^{(h)} \xi
$$

We note the following lemma. 
Lemma 2.5.2. Let $M \in \mathcal{O}_{\text {int }}^{i}$ be an irreducible $\mathbf{U}(i)$ module of highest weight $m \in \mathbb{Z}_{\geq 0}$. Let $\eta \in M_{m}$ satisfy $E_{i} \eta=0$ and let $\xi=F_{i}^{(m)} \eta$.

(a) There is a $\mathbb{Q}(q)^{\pi}$-linear map $\omega: M \longrightarrow M$ defined by $\omega(\eta)=\pi_{i}^{\left(\begin{array}{c}m \\ 2\end{array}\right)} \xi$, $\omega(\xi)=\eta$, and $\omega(u . \eta)=\omega(u) \cdot \omega(\eta)$ for all $u \in \mathbf{U}(i)$. Moreover, $\omega^{4}=1$.

(b) There is a $\mathbb{Q}$-linear involution ${ }^{-}: M \longrightarrow M$ defined by $\bar{q}=\pi q^{-1}, \bar{\pi}=\pi$, $\bar{\eta}=\eta$ and $\overline{u . \eta}=\bar{u} . \bar{\eta}$ for all $u \in \mathbf{U}(i)$.

Using the semisimplicity of the category $\mathcal{O}_{\text {int }}^{i}$, we obtain the following corollary.

Corollary 2.5.3. Let $M \in \mathcal{O}_{\text {int }}^{i}$.

(a) There is a $\mathbb{Q}(q)^{\pi}$-linear map $\omega: M \longrightarrow M$ such that $\omega(u \eta)=\omega(u) \omega(\eta)$ for all $u \in \mathbf{U}(i)$ and $\eta \in M$. Moreover, $\omega^{4}=1$.

(b) There is a $\mathbb{Q}$-linear involution $-: M \longrightarrow M$ defined by $\bar{q}=\pi q^{-1}, \bar{\pi}=\pi$, and $\overline{u . \eta}=\bar{u} . \bar{\eta}$ for all $u \in \mathbf{U}(i)$ and $\eta \in M$.

Note that there are many possible choices of such maps for an arbitrary $M \in \mathcal{O}_{\text {int }}^{i}$, but we shall not need a particular choice.

2.6. Higher Serre Relations. The higher Serre relations were examined in detail in [CHW1, §4], and we will recall the essential definitions and results. To begin, for $i, j \in I$, and $n, m \geq 0$, set

$$
p(n, m ; i, j)=m n p(i) p(j)+\left(\begin{array}{c}
m \\
2
\end{array}\right) p(i)
$$

and, for $i \neq j$, define the elements

$$
\begin{aligned}
e_{i, j ; n, m} & =\sum_{r+s=m}(-1)^{r} \pi_{i}^{p(n, r ; i, j)}\left(\pi_{i} q_{i}\right)^{-r\left(n a_{i j}+m-1\right)} E_{i}^{(r)} E_{j}^{(n)} E_{i}^{(s)}, \\
e_{i, j ; n, m}^{\prime} & =\sum_{r+s=m}(-1)^{r} \pi_{i}^{p(n, r ; i, j)} q_{i}^{-r\left(n a_{i j}+m-1\right)} E_{i}^{(s)} E_{j}^{(n)} E_{i}^{(r)}, \\
f_{i, j ; n, m} & =\sum_{r+s=m}(-1)^{r} \pi_{i}^{p(n, r ; i, j)}\left(\pi_{i} q_{i}\right)^{r\left(n a_{i j}+m-1\right)} F_{i}^{(s)} F_{j}^{(n)} F_{i}^{(r)}, \\
f_{i, j ; n, m}^{\prime} & =\sum_{r+s=m}(-1)^{r} \pi_{i}^{p(n, r ; i, j)} q_{i}^{r\left(n a_{i j}+m-1\right)} F_{i}^{(r)} F_{j}^{(n)} F_{i}^{(s)} .
\end{aligned}
$$

When there is no chance of confusion, we will abbreviate $e_{i, j ; n, m}=e_{n, m}$, etc. Note that we have the equalities

$$
e_{n, m}^{\prime}=\sigma\left(e_{n, m}\right), \quad f_{n, m}^{\prime}=\sigma\left(f_{n, m}\right), \quad e_{n, m}=\omega\left(\overline{f_{n, m}}\right), \quad \text { and } \quad e_{n, m}^{\prime}=\omega\left(\overline{f_{n, m}^{\prime}}\right) .
$$

The following results were proved in [CHW1, §4].

Lemma 2.6.1. The following statements hold:

(a)

$$
\begin{aligned}
E_{i}^{(N)} e_{n, m}=\sum_{k=0}^{N}( & -1)^{k} q_{i}^{N\left(n a_{i j}+2 m\right)+(N-1) k} \pi_{i}^{N(n p(j)+m)+\left(\begin{array}{c}
k \\
2
\end{array}\right)} \\
& \times\left[\begin{array}{c}
m+k \\
k
\end{array}\right]_{i} e_{n, m+k} E_{i}^{(N-k)}
\end{aligned}
$$


(b)

$$
\begin{aligned}
F_{i}^{(M)} e_{n, m}=\sum_{h=0}^{M}(-1)^{h} q_{i}^{-(M-1) h} \pi_{i}^{M(m+n p(j))+(M-m) h} \\
\times\left[\begin{array}{c}
-n a_{i j}-m+h \\
h
\end{array}\right]_{i} K_{i}^{-h} e_{n, m-h} F_{i}^{(M-h)} .
\end{aligned}
$$

(c) If $m>-n a_{i j}$, then $e_{i, j ; n, m}=0$.

\section{BRAID GROUP OPERATORS}

We shall now define certain operators on $\mathbf{U}$ and its integrable modules. These operators are generalizations of Lusztig's braid operators on quantum groups; see L]. Many of our results are direct generalizations of Lusztig's results in loc. cit. to the quantum covering group setting.

3.1. The symmetries $T_{i}$ and $T_{i}^{-1}$ of category $\mathcal{O}$. Fix $i \in I$. Let $M \in \mathcal{O}_{\text {int }}^{i}$. We define the $\mathbb{Q}(q)^{\pi}$-linear maps $T_{i}^{\prime}, T_{i}^{\prime \prime}: M \longrightarrow M$ by

$$
\begin{aligned}
T_{i}^{\prime}(z) & =\sum_{\substack{a, b, c \geq 0 \\
a-b+c=n}}(-1)^{b} \pi_{i}^{c} q_{i}^{-a c+b} \tilde{J}_{i}^{c} F_{i}^{(a)} E_{i}^{(b)} F_{i}^{(c)} z ; \\
T_{i}^{\prime \prime}(z) & =\sum_{\substack{a, b, c \geq 0 \\
-a+b-c=n}}(-1)^{b} \pi_{i}^{a c+c+\left(\begin{array}{c}
n \\
2
\end{array}\right)} q_{i}^{a c-b} \tilde{J}_{i}^{a} E_{i}^{(a)} F_{i}^{(b)} E_{i}^{(c)} z
\end{aligned}
$$

when $z \in M_{n}$. We observe that

$$
p\left(T_{i}^{\prime}(z)\right)=p\left(T_{i}^{\prime \prime}(z)\right)=p(z)+n p(i) .
$$

Remark 3.1.1. Let $M \in \mathcal{O}^{i}$. For $X \in \mathbf{U}(i)$, define the formal power series

$$
\exp (X)=\sum_{t} q_{i}^{-\left(\begin{array}{c}
t \\
2
\end{array}\right)} \frac{X^{t}}{[t]^{!}}
$$

Then $\exp (X)$ defines an operator on any module for which the action of $X$ is locally nilpotent. Further define $q^{\left(\begin{array}{c}\alpha_{i}^{\vee} \\ 2\end{array}\right)}: M \rightarrow M$ via

$$
q^{\left(\begin{array}{c}
\alpha_{i}^{\vee} \\
2
\end{array}\right)}(m)=q_{i}^{\left(\begin{array}{c}
n \\
2
\end{array}\right)} m \text { for } m \in M_{n}
$$

It can be shown that $T_{i}^{\prime}=\exp \left(q_{i}^{-1} F_{i} \tilde{K}_{i}\right) \exp \left(-E_{i}\right) \exp \left(\pi_{i} q_{i} F_{i} \tilde{J}_{i} \tilde{K}_{i}\right) q^{\left(\begin{array}{c}\alpha_{i}^{\vee} \\ 2\end{array}\right)}$, cf. Sai]. 2.5 .2 .

We can relate the maps $T_{i}^{\prime}$ and $T_{i}^{\prime \prime}$ using the module automorphisms from Lemma

Lemma 3.1.2. Let $M \in \mathcal{O}_{\text {int }}^{i}$. Then for $z \in M_{n}$,

(a) $\omega^{2}\left(T_{i}^{\prime}\left(\omega^{2}(z)\right)\right)=T_{i}^{\prime}(z)$,

(b) $T_{i}^{\prime \prime}(z)=\pi_{i}^{\left(\begin{array}{c}n+1 \\ 2\end{array}\right)} \overline{\omega\left(T_{i}^{\prime}\left(\omega^{-1}(\bar{z})\right)\right)}=\pi_{i}^{\left(\begin{array}{c}n+1 \\ 2\end{array}\right)} \overline{\omega^{-1}\left(T_{i}^{\prime}(\omega(\bar{z}))\right)}$,

(c) $T_{i}^{\prime}(z)=\pi_{i}^{\left(\begin{array}{c}n+1 \\ 2\end{array}\right)} \overline{\omega\left(T_{i}^{\prime \prime}\left(\omega^{-1}(\bar{z})\right)\right)}=\pi_{i}^{\left(\begin{array}{c}n+1 \\ 2\end{array}\right)} \overline{\omega^{-1}\left(T_{i}^{\prime \prime}(\omega(\bar{z}))\right)}$. 
Proof. Assume $z \in M_{n}$. Then $\bar{z} \in M_{n}$, so

$$
\begin{aligned}
& \overline{\omega\left(T_{i}^{\prime}\left(\omega^{-1}(\bar{z})\right)\right)}=\overline{\omega\left(\sum_{\substack{a, b, c \geq 0 \\
a-b+c=n}}(-1)^{b} \pi_{i}^{c} q_{i}^{-a c+b} \tilde{J}_{i}^{c} F_{i}^{(a)} E_{i}^{(b)} F_{i}^{(c)} \omega^{-1}(\bar{z})\right)} \\
& =\overline{\sum_{\substack{a, b, c \geq 0 \\
a-b+c=n}}(-1)^{b} \pi_{i}^{c} q_{i}^{-a c+b} J_{i}^{c} E_{i}^{(a)} \pi_{i}^{b} \tilde{J}_{i}^{b} F_{i}^{(b)} E_{i}^{(c)} \bar{z}} \\
& =\sum_{\substack{a, b, c \geq 0 \\
a-b+c=n}}(-1)^{b} \pi_{i}^{a c+c} q_{i}^{-a c+b} \tilde{J}_{i}^{b+c} E_{i}^{(a)} F_{i}^{(b)} E_{i}^{(c)} z \\
& =\pi_{i}^{\left(\begin{array}{c}
n+1 \\
2
\end{array}\right)} T_{i}^{\prime \prime}(z) .
\end{aligned}
$$

In the last line, we have used the fact that $\left.\tilde{J}_{i}^{b+c}\right|_{M_{n}}=\left.\pi_{i}^{n} \tilde{J}_{i}^{a}\right|_{M_{n}}$. This proves the first equality in (b).

Next, using the definition of $\omega$, we compute

$$
\begin{aligned}
\omega^{2}\left(T_{i}^{\prime}\left(\omega^{2}(z)\right)\right) & =\omega^{2}\left(\sum_{\substack{a, b, c \geq 0 \\
a-b+c=n}}(-1)^{b} \pi_{i}^{c} q_{i}^{-a c+b} \tilde{J}_{i}^{c} F_{i}^{(a)} E_{i}^{(b)} F_{i}^{(c)} \omega^{2}(z)\right) \\
& =\sum_{\substack{a, b, c \geq 0 \\
a-b+c=n}}(-1)^{b} \pi_{i}^{c} q_{i}^{-a c+b} \tilde{J}_{i}^{c}\left(\pi_{i}^{a} \tilde{J}_{i}^{a} F_{i}^{(a)}\right)\left(\pi_{i}^{b} \tilde{J}_{i}^{b} E_{i}^{(b)}\right)\left(\pi_{i}^{c} \tilde{J}_{i}^{c} F_{i}^{(c)}\right) z \\
& =\sum_{\substack{a, b, c \geq 0 \\
a-b+c=n}}(-1)^{b} \pi_{i}^{c} q_{i}^{-a c+b} \tilde{J}_{i}^{c} F_{i}^{(a)} E_{i}^{(b)} F_{i}^{(c)}\left(\pi_{i}^{n} \tilde{J}_{i}^{n}\right) z
\end{aligned}
$$

Part (a) follows since $\pi_{i}^{n} \tilde{J}_{i}^{n} z=\pi_{i}^{n}\left(\pi_{i}^{n}\right)^{n} z=\pi_{i}^{n(n+1)} z=z$.

The second equality in (b) now follows. Finally, (c) follows from (a) and (b) since $\omega$ commutes with the bar involution.

The symmetries $T_{i}^{\prime}$ and $T_{i}^{\prime \prime}$ can be computed explicitly on each simple module of $\mathcal{O}^{i}$. In particular, we have the following lemma.

Lemma 3.1.3. Let $M \in \mathcal{O}^{i}$, and $m \in \mathbb{Z}_{\geq 0}$. For $k, h \geq 0$ such that $k+h=m$,

(a) If $\eta \in M_{m}$ satisfies $E_{i} \eta=0$, then

$$
T_{i}^{\prime}\left(F_{i}^{(k)} \eta\right)=(-1)^{k} \pi_{i}^{m k+\left(\begin{array}{c}
k+1 \\
2
\end{array}\right)} q_{i}^{h k+k} F_{i}^{(h)} \eta
$$

(b) If $\xi \in M_{-m}$ satisfies $F_{i} \xi=0$, then

$$
T_{i}^{\prime \prime}\left(E_{i}^{(k)} \xi\right)=(-1)^{k} \pi_{i}^{m h+\left(\begin{array}{c}
h+1 \\
2
\end{array}\right)} q_{i}^{-h k-k} E_{i}^{(h)} \xi
$$


Proof. First note that (b) follows from (a). Indeed, observe that $E_{i}^{(k)} \xi \in M_{k-h}$, so by Lemmas 2.5 .2 and 3.1 .2 .

$$
\begin{aligned}
T_{i}^{\prime \prime}\left(E_{i}^{(k)} \xi\right) & =\pi_{i}^{\left(\begin{array}{c}
k-h+1 \\
2
\end{array}\right) \overline{\omega\left(T_{i}^{\prime}\left(\omega^{-1}\left(\overline{E_{i}^{(k)} \xi}\right)\right)\right.}} \\
& =\pi_{i}^{\left(\begin{array}{c}
k-h+1 \\
2
\end{array}\right)+\left(\begin{array}{c}
k-h \\
2
\end{array}\right)} \overline{\omega\left(T_{i}^{\prime}\left(F_{i}^{(k)} \bar{\eta}\right)\right)} \\
& =\pi_{i}^{\left(\begin{array}{c}
k-h+1 \\
2
\end{array}\right)+\left(\begin{array}{c}
k-h \\
2
\end{array}\right)} \overline{\omega\left((-1)^{k} \pi_{i}^{m k+\left(\begin{array}{c}
k+1 \\
2
\end{array}\right)} q_{i}^{h k+k} F_{i}^{(h)} \bar{\eta}\right)} \\
& =(-1)^{k} \pi_{i}^{\left(\begin{array}{c}
k-h+1 \\
2
\end{array}\right)+m k+\left(\begin{array}{c}
k+1 \\
2
\end{array}\right)}\left(\pi_{i} q_{i}\right)^{-h k-k} E_{i}^{(h)} \xi .
\end{aligned}
$$

Then part (b) now follows from part (a) and the congruence

$$
\left(\begin{array}{c}
k-h+1 \\
2
\end{array}\right)+m k+\left(\begin{array}{c}
k+1 \\
2
\end{array}\right)+h k+k \equiv m h+\left(\begin{array}{c}
h+1 \\
2
\end{array}\right) \quad(\bmod 2) .
$$

It remains to prove (a). Assume $a-b+c=m-2 k$. Using Lemma 2.4.5 we have

$$
\begin{aligned}
F_{i}^{(a)} E_{i}^{(b)} F_{i}^{(c)} F_{i}^{(k)} \eta & =\left[\begin{array}{c}
c+k \\
c
\end{array}\right]_{i} F_{i}^{(a)} E_{i}^{(b)} F_{i}^{(c+k)} \eta \\
& =\sum_{t \geq 0}\left[\begin{array}{c}
c+k \\
c
\end{array}\right]_{i}\left[\begin{array}{c}
b-c+h \\
t
\end{array}\right]_{i} \pi_{i}^{b(c+k)+\left({ }^{t+1}\right)} F_{i}^{(a)} F_{i}^{(c+k-t)} E_{i}^{(b-t)} \eta
\end{aligned}
$$

By assumption, $E_{i}^{(b-t)} \eta \neq 0$ only when $b=t$. Hence,

$$
\begin{aligned}
& F_{i}^{(a)} E_{i}^{(b)} F_{i}^{(c)} F_{i}^{(k)} \eta=\left[\begin{array}{c}
c+k \\
c
\end{array}\right]_{i}\left[\begin{array}{c}
b-c+h \\
b
\end{array}\right]_{i} \pi_{i}^{b(c+k)+\left(\begin{array}{c}
b+1 \\
2
\end{array}\right)} F_{i}^{(a)} F_{i}^{(c+k-b)} \eta
\end{aligned}
$$

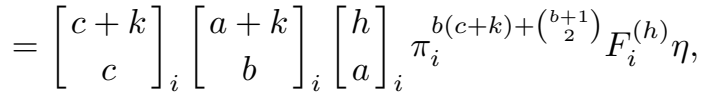

where we have used $a-b+c=h-k$ to make the substitution $b-c+h=a+k$ in the last line. Since $\tilde{J}_{i}^{c}$ acts on $F_{i}^{(k)} \eta$ as multiplication by $\pi_{i}^{c(h-k)}$, we see that it suffices to show that

$$
\begin{aligned}
(-1)^{k} \pi_{i}^{m k+\left(\begin{array}{c}
k+1 \\
2
\end{array}\right)} q_{i}^{h k+k}= & \sum_{\substack{a, b, c \geq 0 \\
a-b+c=h-k}}(-1)^{b} \pi_{i}^{b(c+k)+\left(\begin{array}{c}
b+1 \\
2
\end{array}\right)+c+c(h-k)} q_{i}^{-a c+b} \\
& \times\left[\begin{array}{c}
c+k \\
c
\end{array}\right]_{i}\left[\begin{array}{c}
a+k \\
b
\end{array}\right]_{i}\left[\begin{array}{c}
h \\
a
\end{array}\right]_{i}
\end{aligned}
$$

The equality $(\star)$ can be proven directly by an argument similar to the $\pi=$ 1 specialization of $(\star)$ given in the proof of [ $\mathrm{L}$, Proposition 5.2.21] using (2.8). Alternatively, $(\star)$ can be deduced from the $\pi=1$ case by rewriting the identity in $\pi q^{2}$; see the proof of [CHW2, Lemma 7.2] for a similar deduction.

In particular, we arrive at the following relation between $T_{i}^{\prime}$ and $T_{i}^{\prime \prime}$ as maps on modules in $\mathcal{O}^{i}$.

Proposition 3.1.4. We have $T_{i}^{\prime} T_{i}^{\prime \prime}=T_{i}^{\prime \prime} T_{i}^{\prime}=1: M_{n} \longrightarrow M_{n}$. 
Proof. Let $m=h+k$, and $\eta$ be as in Lemma 3.1.3. Define $\xi=F_{i}^{(m)} \eta$ so that by Corollary 2.5.1 $\pi_{i}^{(h-k) h+\left(\begin{array}{c}h+1 \\ 2\end{array}\right)} F_{i}^{(k)} \eta=E_{i}^{(h)} \xi$, and $F_{i}^{(h)} \eta=\pi_{i}^{(h-k) k+\left(\begin{array}{c}k+1 \\ 2\end{array}\right)} E_{i}^{(k)} \xi$. Then, using Lemma 3.1.3, we have

$$
\begin{aligned}
& T_{i}^{\prime \prime} T_{i}^{\prime}\left(F_{i}^{(k)} \eta\right)=T_{i}^{\prime \prime}\left((-1)^{k} \pi_{i}^{m k+\left(\begin{array}{c}
{ }^{k+1} \\
2
\end{array}\right)} q_{i}^{h k+k} F_{i}^{(h)} \eta\right) \\
& =(-1)^{k} \pi_{i}^{m k+\left(\begin{array}{c}
k+1 \\
2
\end{array}\right)} q_{i}^{h k+k} T_{i}^{\prime \prime}\left(\pi_{i}^{m k+\left(\begin{array}{c}
k+1 \\
2
\end{array}\right)} E_{i}^{(k)} \xi\right) \\
& =(-1)^{k} q_{i}^{h k+k}(-1)^{k} \pi_{i}^{m h+\left(\begin{array}{c}
h+1 \\
2
\end{array}\right)} q_{i}^{-h k-k} E_{i}^{(h)} \xi \\
& =\pi_{i}^{m h+\left(\begin{array}{c}
h+1 \\
2
\end{array}\right)} \pi_{i}^{m h+\left(\begin{array}{c}
h+1 \\
2
\end{array}\right)} F_{i}^{(k)} \eta \\
& =F_{i}^{(k)} \eta \text {. }
\end{aligned}
$$

Now, $M$ is generated by vectors of the form $F_{i}^{(k)} \eta$ as above, so $T_{i}^{\prime \prime} T_{i}^{\prime}=1$. The remaining identity $T_{i}^{\prime} T_{i}^{\prime \prime}=1$ can be deduced in the same fashion.

In light of this result, we shall henceforth use the following notations:

$$
T_{i}=T_{i}^{\prime} \quad \text { and } \quad T_{i}^{-1}=T_{i}^{\prime \prime} .
$$

Lemma 3.1.5. For $z \in M_{t}$,

$$
\overline{T_{i}(\bar{z})}=(-1)^{t} \pi_{i}^{\left(\begin{array}{c}
t \\
2
\end{array}\right)} q_{i}^{t} T_{i}^{-1}(z)
$$

Proof. We may assume that $z=F_{i}^{(k)} \eta=\pi_{i}^{m h+\left(\begin{array}{c}h+1 \\ 2\end{array}\right)} E_{i}^{(h)} \xi$, where $m=k+h, \eta, \xi$ are as in Lemma 3.1.3. In this case, $h=k+t$ and we will use the fact that $m \equiv t$ $(\bmod 2)$ throughout the proof. By Lemma 3.1.3.

$$
\begin{aligned}
T_{i}^{-1}(z) & =\pi_{i}^{m h+\left(\begin{array}{c}
h+1 \\
2
\end{array}\right)} T_{i}^{-1}\left(E_{i}^{(h)} \xi\right) \\
& =(-1)^{h} \pi_{i}^{m h+\left(\begin{array}{c}
h+1 \\
2
\end{array}\right)+m k+\left(\begin{array}{c}
k+1 \\
2
\end{array}\right)} q_{i}^{-k h-h} E_{i}^{(k)} \xi \\
& =(-1)^{h} \pi_{i}^{m k+\left(\begin{array}{c}
t \\
2
\end{array}\right)} q_{i}^{-k h-h} E_{i}^{(k)} \xi
\end{aligned}
$$

where, in the last line, we have used

$$
m h+\left(\begin{array}{c}
h+1 \\
2
\end{array}\right)+m k+\left(\begin{array}{c}
k+1 \\
2
\end{array}\right) \equiv m k+\left(\begin{array}{l}
t \\
2
\end{array}\right) .
$$

On the other hand, by Lemma 3.1 .3 .

$$
\begin{aligned}
\overline{T_{i}(\bar{z})} & =\overline{T_{i}\left(F_{i}^{(k)} \bar{\eta}\right)}=\overline{(-1)^{k} \pi_{i}^{m k+\left(\begin{array}{c}
k+1 \\
2
\end{array}\right)} q_{i}^{h k+k} F_{i}^{(h)} \bar{\eta}} \\
& =(-1)^{k} \pi_{i}^{m k+\left(\begin{array}{c}
k+1 \\
2
\end{array}\right)+h k+k} q_{i}^{-h k-k} F_{i}^{(h)} \eta=(-1)^{k} \pi_{i}^{m k} q_{i}^{-h k-k} E_{i}^{(k)} \xi .
\end{aligned}
$$

The result follows.

Lemma 3.1.6. For any $z \in M_{t}$,

(a) $T_{i}\left(F_{i} z\right)=-q_{i}^{t} E_{i} T_{i}(z)$;

(b) $T_{i}^{-1}\left(F_{i} z\right)=-\pi_{i}^{t+1} q_{i}^{-t+2} E_{i} T_{i}^{-1}(z)$;

(c) $T_{i}\left(E_{i} z\right)=-\pi_{i}^{t+1} q_{i}^{-t-2} F_{i} T_{i}(z)$;

(d) $T_{i}^{-1}\left(E_{i} z\right)=-q_{i}^{t} F_{i} T_{i}^{-1}(z)$;

(e) $T_{i}(z) \in M_{-t}$; 
(f) $T_{i}^{-1}(z) \in M_{-t}$.

Proof. Properties (e) and (f) are clear by the definitions of $T_{i}$ and $T_{i}^{-1}$. We also note that (d) follows from (a) and (c) follows from (b) using Proposition 3.1.4. As the proofs of (a) and (b) are entirely similar, we shall only prove (a).

To this end, assume that $z=F_{i}^{(k)} \eta=\pi_{i}^{m h+\left(\begin{array}{c}(h+1) \\ 2\end{array}\right)} E_{i}^{(h)} \xi$, where $m=k+h, \eta, \xi$ are as in Lemma 3.1.3. In this case, $h=k+t$ and we will repeatedly use the fact that $m \equiv t(\bmod 2)$ throughout the proof. Note that if $k=m$, then both sides of both (a) and (b) are zero. Therefore, assume $k<m$ and $h>0$. Then, for (a),

$$
\begin{aligned}
T_{i}\left(F_{i} z\right) & =[k+1]_{i} T_{i}\left(F_{i}^{(k+1)} \eta\right) \\
& =(-1)^{k+1} \pi_{i}^{m(k+1)+\left(\begin{array}{c}
k+2 \\
2
\end{array}\right)} q_{i}^{h(k+1)}[k+1]_{i} F_{i}^{(h-1)} \eta \\
& =(-1)^{k+1} \pi_{i}^{m(k+1)+\left(\begin{array}{c}
k+2 \\
2
\end{array}\right)+m(k+1)+\left(\begin{array}{c}
(k+2 \\
2
\end{array}\right)} q_{i}^{h(k+1)}[k+1]_{i} E_{i}^{(k+1)} \xi \\
& =(-1)^{k+1} q_{i}^{h(k+1)}[k+1]_{i} E_{i}^{(k+1)} \xi,
\end{aligned}
$$

while

$$
\begin{aligned}
E_{i} T_{i}(z) & =E_{i} T_{i}\left(F_{i}^{(k)} \eta\right) \\
& =(-1)^{k} \pi_{i}^{m k+\left({ }_{2}^{k+1}\right)} q_{i}^{(h+1) k} E_{i} F_{i}^{(h)} \eta \\
& =(-1)^{k} \pi_{i}^{m k+\left({ }^{k+1}\right)+m k+\left({ }^{k+1}\right)} q_{i}^{(h+1) k} E_{i} E_{i}^{(k)} \xi \\
& =(-1)^{k} q_{i}^{(h+1) k}[k+1]_{i} E_{i}^{(k+1)} \xi .
\end{aligned}
$$

Therefore, part (a) follows since $h=k+t$.

3.2. Braid operators on $\mathcal{O}_{\text {int }}$. Now, assume that $M \in \mathcal{O}_{\text {int }}$. Then $M$ can be regarded as an object of $\mathcal{O}_{\text {int }}^{i}$ for each $i \in I$, and we obtain an action of the symmetries

$$
T_{i}, T_{i}^{-1}: M \longrightarrow M
$$

We call these the braid operators of $\mathcal{O}_{\text {int }}$. We note that $T_{i}, T_{i}^{-1}$ are not homogeneous with respect to the $\mathbb{Z} / 2 \mathbb{Z}$-grading on $M$; however, they are homogeneous on each weight space. To wit, for $\lambda \in P$ and homogeneous $m \in M_{\lambda}$, we have that

$$
p\left(T_{i}(m)\right)=p\left(T_{i}^{-1}(m)\right) \equiv p(m)+p(i)\left\langle\alpha_{i}^{\vee}, \lambda\right\rangle \quad(\bmod 2)
$$

Lemma 3.2.1. Let $M \in \mathcal{O}_{\text {int }}$, and let $z \in M$. Fix $\mu \in P^{\vee}$, and let

$$
\nu=s_{\alpha_{i}^{\vee}}(\mu)=\mu-\left\langle\mu, \alpha_{i}\right\rangle \alpha_{i}^{\vee} \in P^{\vee} .
$$

Then,

(a) $T_{i}^{-1}\left(K_{\nu} z\right)=K_{\mu} T_{i}^{-1}(z)$ and $T_{i}^{-1}\left(J_{\nu} z\right)=J_{\mu} T_{i}^{-1}(z)$;

(b) $T_{i}\left(K_{\nu} z\right)=K_{\mu} T_{i}(z)$ and $T_{i}\left(J_{\nu} z\right)=J_{\mu} T_{i}(z)$;

Moreover,

(c) $T_{i}\left(\tilde{J}_{\nu} z\right)=\tilde{J}_{\nu} T_{i}(z)$;

(d) $T_{i}^{-1}\left(\tilde{J}_{\nu} z\right)=\tilde{J}_{\nu} T_{i}^{-1}(z)$.

Proof. Parts (a) and (b) are proved exactly as in [L, Proposition 5.2.6]. The main point is that if $z$ is a weight vector, say $z \in M_{\lambda}$, then $T_{i}(z), T_{i}^{-1}(z) \in M_{\lambda-\left\langle\alpha_{i}^{\vee}, \lambda\right\rangle \alpha_{i}}$ 
by Lemma 3.1.6(e),(f). It is left to observe that $K_{\mu}$ (respectively, $J_{\mu}$ ) acts on the $\lambda-\left\langle\alpha_{i}^{\vee}, \lambda\right\rangle \alpha_{i}$ weight space as multiplication by $q^{\star}$ (respectively, $\pi^{\star}$ ), where

$$
\star=\langle\mu, \lambda\rangle-\left\langle\alpha_{i}^{\vee}, \lambda\right\rangle\left\langle\mu, \alpha_{i}\right\rangle=\langle\nu, \lambda\rangle .
$$

Finally, we prove (c) and (d). For this, note that $\tilde{J}_{\mu}=J_{\tilde{\mu}}$ by definition and $\left\langle\tilde{\mu}, \alpha_{i}\right\rangle \in 2 \mathbb{Z}$, which in turn implies that $\langle\mu, \lambda\rangle \equiv\langle\nu, \lambda\rangle(\bmod 2)$.

Corollary 3.2.2. Let $M \in \mathcal{O}_{\text {int }}$. The maps $T_{i}, T_{i}^{-1}: M \rightarrow M$ restrict to bijections between $M_{\lambda}$ and $M_{\lambda-\left\langle\alpha_{i}^{\vee}, \lambda\right\rangle \alpha_{i}}$.

Recall the elements $e_{n, m ; i, j}, e_{n, m ; i, j}^{\prime}, f_{n, m ; i, j}$, and $f_{n, m ; i, j}^{\prime}$ defined by (2.31)(2.34).

Lemma 3.2.3. Let $i, j \in I$ and assume that $i \neq j$. Let $M$ be any object in $\mathcal{O}_{\mathrm{int}}$ and let in $M$. We have

(a) $T_{i}^{-1}\left(e_{i, j ; n,-n a_{i j}} z\right)=\pi_{i}^{\left({ }^{n a_{i j}}\right)} \tilde{J}_{i}^{n p(j)} E_{j}^{(n)} T_{i}^{-1}(z)$;

(b) $T_{i}\left(e_{i, j ; n,-n a_{i j}}^{\prime} z\right)=\pi_{i}^{\left({ }^{n a_{i j}}\right)} \tilde{J}_{i}^{n p(j)} E_{j}^{(n)} T_{i}(z)$;

(c) $T_{i}^{-1}\left(f_{i, j ; n,-n a_{i j}} z\right)=\tilde{J}_{i}^{n p(j)} F_{j}^{(n)} T_{i}^{-1}(z)$;

(d) $T_{i}\left(f_{i, j ; n,-n a_{i j}}^{\prime} z\right)=\tilde{J}_{i}^{n p(j)} F_{j}^{(n)} T_{i}(z)$.

Proof. As before, we write $e_{n, m}=e_{i, j ; n, m}$. We may assume $z \in M_{\lambda}$ for some $\lambda \in P$. Then, $e_{n,-n a_{i j}} z \in M_{\lambda-n a_{i j} \alpha_{i}+n \alpha_{j}}$. Let

$$
p=\left\langle\alpha_{i}^{\vee}, \lambda\right\rangle \quad \text { and } \quad p^{\prime}=\left\langle\alpha_{i}^{\vee}, \lambda-n a_{i j} \alpha_{i}+n \alpha_{j}\right\rangle=p-n a_{i j} .
$$

Note that, since $\tilde{J}_{i}^{a} e_{n,-n a_{i j}} z=\pi_{i}^{a p^{\prime}} e_{n,-n a_{i j}} z$,

$$
T_{i}^{-1}\left(e_{n,-n a_{i j}} z\right)=\sum_{\substack{a, b, c \geq 0 \\
-a+b-c=p^{\prime}}}(-1)^{b} \pi_{i}^{a c+c+\left(\begin{array}{c}
p^{\prime} \\
2
\end{array}\right)+a p^{\prime}} q_{i}^{a c-b} E_{i}^{(a)} F_{i}^{(b)} E_{i}^{(c)} e_{n,-n a_{i j}} z .
$$

By Lemma 2.6.1, we have that

$$
E_{i}^{(c)} e_{n,-n a_{i j}}=q_{i}^{-c n a_{i j}} \pi_{i}^{c n p(j)} e_{n,-n a_{i j}} E_{i}^{(c)} .
$$

Therefore, using Lemma 2.6.1(b), then (a), we deduce that

$$
\begin{aligned}
& E_{i}^{(a)} F_{i}^{(b)} E_{i}^{(c)} e_{n,-n a_{i j}} z=q_{i}^{-c n a_{i j}} \pi_{i}^{c n p(j)} E_{i}^{(a)} F_{i}^{(b)} e_{n,-n a_{i j}} E_{i}^{(c)} z \\
& =q_{i}^{-c n a_{i j}} \pi_{i}^{c n p(j)} E_{i}^{(a)} \sum_{b^{\prime}=0}^{b}(-1)^{b^{\prime}} q_{i}^{-(b-1) b^{\prime}} \pi_{i}^{b n p(j)+b b^{\prime}} K_{i}^{-b^{\prime}} e_{n,-n a_{i j}-b^{\prime}} F_{i}^{\left(b-b^{\prime}\right)} E_{i}^{(c)} z \\
& =\sum_{b^{\prime}=0}^{b} \sum_{a^{\prime}=0}^{a}(-1)^{b^{\prime}+a^{\prime}} q_{i}^{\mathbf{A} 0} \pi_{i}^{\mathbf{2} 0_{0}}\left[\begin{array}{c}
-n a_{i j}-b^{\prime}+a^{\prime} \\
a^{\prime}
\end{array}\right]_{i} e_{n,-n a_{i j}-b^{\prime}+a^{\prime}} E_{i}^{\left(a-a^{\prime}\right)} F_{i}^{\left(b-b^{\prime}\right)} E_{i}^{(c)} z
\end{aligned}
$$

where

$$
\begin{aligned}
\mathbf{\phi}_{0} & =-c n a_{i j}-(b-1) b^{\prime}-b^{\prime}\left(p^{\prime}+2(c-b)\right)+a\left(n a_{i j}-2 n a_{i j}-2 b^{\prime}\right)+(a-1) a^{\prime} \\
& =-(a+c) n a_{i j}-2 a b^{\prime}+b b^{\prime}-2 c b^{\prime}-b^{\prime} p^{\prime}+b^{\prime}+a a^{\prime}-a^{\prime},
\end{aligned}
$$

and

$$
\boldsymbol{\beta}_{0} \equiv(-a+b-c) n p(j)+(a+b) b^{\prime}+\left(\begin{array}{c}
a^{\prime} \\
2
\end{array}\right) \quad(\bmod 2)
$$


Introduce the variables $a^{\prime \prime}=a-a^{\prime}$ and $b^{\prime \prime}=b-b^{\prime}$. Then, summing over $a, b, c \geq 0$ such that $-a+b-c=p^{\prime}$ and, using the relation $n a_{i j}=a^{\prime}+a^{\prime \prime}-b^{\prime}-b^{\prime \prime}+c+p$, we obtain

$$
\begin{aligned}
& T_{i}^{-1}\left(e_{n,-n a_{i j}} z\right)=\sum_{\substack{a, b, c \geq 0 \\
-a+b-c=p^{\prime}}} \sum_{\substack{a^{\prime}, a^{\prime \prime} \geq 0 \\
a^{\prime \prime}+a^{\prime}=a}} \sum_{\substack{b^{\prime}, b^{\prime \prime} \geq 0 \\
b^{\prime \prime}+b^{\prime}=b}}(-1)^{b^{\prime \prime}+a^{\prime}} q_{i}^{\mathbf{\omega}} \pi_{i}^{\mathbf{\alpha}}\left[\begin{array}{c}
-a^{\prime \prime}+b^{\prime \prime}-c-p \\
a^{\prime}
\end{array}\right]_{i} \\
& \times e_{n,-a^{\prime \prime}+b^{\prime \prime}-c-p} E_{i}^{\left(a^{\prime \prime}\right)} F_{i}^{\left(b^{\prime \prime}\right)} E_{i}^{(c)} z,
\end{aligned}
$$

where

$$
\mathbf{A}=a c-b+\boldsymbol{\Lambda}_{0}=a^{\prime}\left(-1+b^{\prime \prime}-a^{\prime \prime}-c-p\right)+\left(a^{\prime \prime} c-b^{\prime \prime}\right)+\left(a^{\prime \prime}+c\right)\left(-a^{\prime \prime}+b^{\prime \prime}-c-p\right),
$$

and

$$
\begin{aligned}
\boldsymbol{\phi}= & a c+c+\left(\begin{array}{c}
p^{\prime} \\
2
\end{array}\right)+a p^{\prime}+\boldsymbol{k}_{0} \\
\equiv & {\left[\left(\begin{array}{c}
n a_{i j} \\
2
\end{array}\right)+p n p(j)\right]+\left(a^{\prime \prime} c+c+\left(\begin{array}{l}
p \\
2
\end{array}\right)+a^{\prime \prime} p\right) } \\
& +\left(a^{\prime} c+a^{\prime} p+(a+b) b^{\prime}\right)+\left(\begin{array}{c}
a^{\prime} \\
2
\end{array}\right) .
\end{aligned}
$$

Using the congruence $a+b+c \equiv p(\bmod 2)$, we can rewrite

$$
a^{\prime} c+a^{\prime} p+(a+b) b^{\prime} \equiv(a+b)\left(a^{\prime}+b^{\prime}\right) \equiv(c+p)\left(a^{\prime \prime}+b^{\prime \prime}+c+p\right) .
$$

Hence,

$$
\boldsymbol{a} \equiv\left(\begin{array}{c}
n a_{i j} \\
2
\end{array}\right)+p n p(j)+a^{\prime \prime} c+c+\left(\begin{array}{l}
p \\
2
\end{array}\right)+a^{\prime \prime} p+(c+p)\left(a^{\prime \prime}+b^{\prime \prime}+c+p\right)+\left(\begin{array}{c}
a^{\prime} \\
2
\end{array}\right) \text {. }
$$

By Lemma 2.6.1 and the definitions, $e_{n,-a^{\prime \prime}+b^{\prime \prime}-c-p}=0$ unless $0 \leq-a^{\prime \prime}+b^{\prime \prime}-c-p \leq$ $-n a_{i j}$. We may therefore add this condition without changing the sum. Now, from the equation $-a+b-c=p-n a_{i j}$ and the previous inequality, we deduce that the sum involving $b^{\prime}$ is redundant, as $b^{\prime} \geq 0$ is determined by $a^{\prime}, a^{\prime \prime}, b^{\prime \prime}, c$ :

$$
b^{\prime}=a^{\prime}+a^{\prime \prime}-b^{\prime \prime}+c+p-n a_{i j} \geq 0 .
$$

Therefore, the sum (3.5) becomes

$$
\begin{aligned}
\pi_{i}^{\boldsymbol{\phi}^{\prime \prime \prime}} \sum_{a^{\prime \prime}, b^{\prime \prime}, c^{\prime \prime}}(-1)^{b^{\prime \prime}} \pi_{i}^{\mathbf{Q}^{\prime \prime}} q_{i}^{\mathbf{\phi}^{\prime \prime}} & \left(\sum_{a^{\prime} \geq 0}(-1)^{a^{\prime}} \pi_{i}^{\mathbf{Q}^{\prime}} q_{i}^{\mathbf{\phi}^{\prime}}\left[\begin{array}{c}
\left.-a^{\prime \prime}+b^{\prime \prime}-c-p\right]_{i} \\
a^{\prime}
\end{array}\right)\right. \\
& \times e_{n,-a^{\prime \prime}+b^{\prime \prime}-c-p} E_{i}^{\left(a^{\prime \prime}\right)} F_{i}^{\left(b^{\prime \prime}\right)} E_{i}^{(c)} z,
\end{aligned}
$$

where the first sum is over $a^{\prime \prime}, b^{\prime \prime}, c \geq 0$ with $0 \leq-a^{\prime \prime}+b^{\prime \prime}-c-p \leq-n a_{i j}$,

$$
\begin{gathered}
\mathbf{Q}^{\prime}=a^{\prime}\left(-1+b^{\prime \prime}-a^{\prime \prime}-c-p\right) \quad \text { and } \quad \mathbf{Q}^{\prime \prime}=\left(a^{\prime \prime} c-b^{\prime \prime}\right)+\left(a^{\prime \prime}+c\right)\left(-a^{\prime \prime}+b^{\prime \prime}-c-p\right), \\
\mathbf{Q}^{\prime}=\left(\begin{array}{c}
a^{\prime} \\
2
\end{array}\right), \quad \boldsymbol{\beta}^{\prime \prime}=a^{\prime \prime} c+c+\left(\begin{array}{l}
p \\
2
\end{array}\right)+a^{\prime \prime} p+(c+p)\left(a^{\prime \prime}+b^{\prime \prime}+c+p\right),
\end{gathered}
$$

and

$$
\mathbf{q}^{\prime \prime \prime}=\left(\begin{array}{c}
n a_{i j} \\
2
\end{array}\right)+p n p(j)
$$


Now, we deduce from (2.9) that the sum over $a^{\prime}$ in (3.6) is 0 unless $-a^{\prime \prime}+b^{\prime \prime}-c-p=$ 0 . Summarizing the above computation, we have

$$
\begin{aligned}
& T_{i}^{-1}\left(e_{n,-n a_{i j}} z\right)=\pi_{i}^{\boldsymbol{Q}^{\prime \prime \prime}} e_{n, 0} \sum_{\substack{a^{\prime \prime}, b^{\prime \prime}, c^{\prime \prime} \geq 0 \\
-a^{\prime \prime}+b^{\prime \prime}-c=p}}(-1)^{b^{\prime \prime}} \pi_{i}^{a^{\prime \prime} c+c+\left(\begin{array}{c}
p \\
2
\end{array}\right)+a^{\prime \prime} p} q_{i}^{a^{\prime \prime} c-b^{\prime \prime}} E_{i}^{\left(a^{\prime \prime}\right)} F_{i}^{\left(b^{\prime \prime}\right)} E_{i}^{(c)} z \\
& =\pi_{i}^{\boldsymbol{Q}^{\prime \prime \prime}} e_{n, 0} \sum_{\substack{a^{\prime \prime}, b^{\prime \prime}, c^{\prime \prime} \geq 0 \\
-a^{\prime \prime}+b^{\prime \prime}-c=p}}(-1)^{b^{\prime \prime}} \pi_{i}^{a^{\prime \prime} c+c+\left(\begin{array}{l}
p \\
2
\end{array}\right)} q_{i}^{a^{\prime \prime} c-b^{\prime \prime}} \tilde{J}_{i}^{a^{\prime \prime}} E_{i}^{\left(a^{\prime \prime}\right)} F_{i}^{\left(b^{\prime \prime}\right)} E_{i}^{(c)} z \\
& =\pi_{i}^{\left(\begin{array}{c}
n a_{i j} \\
2^{2}
\end{array}\right)} \tilde{J}_{i}^{n p(j)} E_{j}^{(n)} T_{i}^{-1}(z) .
\end{aligned}
$$

This proves (a).

To prove (b), observe that by Lemma 3.1.5.

$$
\left.\overline{T_{i}^{-1}(\bar{z})}=(-1)^{\left\langle\alpha_{i}^{\vee}, \lambda\right\rangle} \pi_{i}^{\left({ }_{i}^{\vee}, \lambda\right\rangle}\right) q_{i}^{\left\langle\alpha_{i}^{\vee}, \lambda\right\rangle} T_{i}(z),
$$

and, due to $(\mathrm{P} 1)$

$$
\left.\overline{T_{i}^{-1}\left(e_{n,-n a_{i j}} \bar{z}\right)}=(-1)^{\left\langle\alpha_{i}^{\vee}, \lambda\right\rangle} \pi_{i}^{\left(\left\langle\alpha_{i}^{\vee}, \lambda\right\rangle+n a_{i j}\right.}\right) q_{i}^{\left\langle\alpha_{i}^{\vee}, \lambda\right\rangle-n a_{i j}} T_{i}\left(\overline{e_{n,-n a_{i j}}} z .\right.
$$

Hence,

$$
T_{i}\left(\overline{e_{n,-n a_{i j}}} z\right)=q_{i}^{-n a_{i j}} \tilde{J}_{i}^{n p(j)} E_{j}^{(n)} T_{i}(z) .
$$

Now, by (P1), $p(i) a_{i j}$ is even, so

$$
e_{n,-n a_{i j}}^{\prime}=\pi_{i}^{\left(-n a_{i j}\right)} q_{i}^{-n a_{i j}} \overline{e_{n,-n a_{i j}}} .
$$

Hence, (b) holds once we observe that $\left(\begin{array}{c}-n a_{i j} \\ 2\end{array}\right) \equiv\left(\begin{array}{c}n a_{i j} \\ 2\end{array}\right)(\bmod 2)$.

Finally, we prove (c) and (d). First note that $e_{n, m}=\omega\left(\overline{f_{n, m}^{\prime}}\right)$ and $e_{n, m}^{\prime}=$ $\omega\left(\overline{f_{n, m}}\right)$. Now, if $z \in M_{\lambda}$, then $f_{n,-n a_{i j}} z, f_{n,-n a_{i j}}^{\prime} z \in M_{\lambda+n a_{i j} \alpha_{i}-n \alpha_{j}}$.

Using part (b) and Lemma $3.1 .2(\mathrm{~b})$,

$$
\begin{aligned}
& \left.T_{i}^{-1}\left(f_{n,-n a_{i j}} z\right)=\pi_{i}^{\left(\left\langle_{i}^{\vee}, \lambda\right\rangle+n a_{i j}+1\right.}\right) \omega^{-1}\left(\overline{T_{i}\left(e_{n,-n a_{i j}}^{\prime} \omega(\bar{z})\right)}\right) \\
& \left.=\pi_{i}^{\left(\begin{array}{c}
n a_{i j} \\
2
\end{array}\right)+\left(\begin{array}{c}
n a_{i j} \\
2
\end{array}\right)} \tilde{J}_{i}^{n p(j)} \omega^{-1}\left(E_{j}^{(n)}\right) \pi_{i}^{\left(\left\langle\alpha_{i}^{\vee}, \lambda\right\rangle+1\right.}\right) \omega^{-1}\left(\overline{T_{i}(\omega(\bar{z}))}\right) .
\end{aligned}
$$

and, by part (a) and Lemma 3.1 .2 (c),

$$
\begin{aligned}
& \left.T_{i}\left(f_{n,-n a_{i j}}^{\prime} z\right)=\pi_{i}^{\left(\left\langle\alpha_{i}^{\vee}, \lambda\right\rangle+n a_{i j}+1\right.}\right) \omega^{-1}\left(\overline{T_{i}^{-1}\left(e_{n,-n a_{i j}} \omega(\bar{z})\right)}\right) \\
& \left.=\pi_{i}^{\left(\begin{array}{c}
n a_{i j} \\
2
\end{array}\right)+\left(\begin{array}{c}
n a_{i j} \\
2
\end{array}\right)} \tilde{J}_{i}^{n p(j)} \omega^{-1}\left(E_{j}^{(n)}\right) \pi_{i}^{\left(\alpha_{i}^{\vee}, \lambda\right\rangle+1}\right) \omega^{-1}\left(\overline{T_{i}^{-1}(\omega(\bar{z}))}\right) .
\end{aligned}
$$

In both cases, we have used condition (P1) to deduce that

$$
\left(\begin{array}{c}
\left\langle\alpha_{i}^{\vee}, \lambda\right\rangle+n a_{i j}+1 \\
2
\end{array}\right) \equiv\left(\begin{array}{c}
\left\langle\alpha_{i}^{\vee}, \lambda\right\rangle+1 \\
2
\end{array}\right)+\left(\begin{array}{c}
n a_{i j} \\
2
\end{array}\right) \quad(\bmod 2) .
$$

This proves (c) and (d). 
3.3. The symmetries $T_{i}$ and $T_{i}^{-1}$ of $\mathbf{U}$. The properties of the braid operators on $\mathcal{O}_{\text {int }}$ allow us to define analogous operators on the quantum group itself. In particular, Lemmas 3.1.6, 3.2.1, and 3.2.3 allow for us to directly generalize the proof of [L, §37.2.3], obtaining the following theorem.

Theorem 3.3.1. (a) For any $u \in \mathbf{U}$, there exists a unique element $u^{\prime} \in \mathbf{U}$ such that $T_{i}\left(u^{\prime} z\right)=u T_{i}(z)$ for any $M \in \mathcal{O}_{\text {int }}$ and any $z \in M$. Moreover, the map $u \mapsto u^{\prime}$ is a $\mathbb{Q}^{\pi}(q)$-algebra automorphism of $\mathbf{U}$, denoted $T_{i}^{-1}$.

(b) For any $u \in \mathbf{U}$, there exists a unique element $u^{\prime \prime} \in \mathbf{U}$ such that $T_{i}^{-1}\left(u^{\prime \prime} z\right)=$ $u T_{i}^{-1}(z)$ for any $M \in \mathcal{O}_{\text {int }}$ and any $z \in M$. Moreover, the map $u \mapsto u^{\prime \prime}$ is $a \mathbb{Q}^{\pi}(q)$-algebra automorphism of $\mathbf{U}$, denoted $T_{i}$.

The automorphisms $T_{i}, T_{i}^{-1}: \mathbf{U} \longrightarrow \mathbf{U}$ are mutually inverse, and defined on the divided powers in the Chevalley generators of $\mathbf{U}$ by the formulae:

$$
\begin{array}{ll}
T_{i}\left(E_{i}^{(n)}\right)=(-1)^{n} \pi_{i}^{n} q_{i}^{n(n-1)} \tilde{J}_{i}^{n} \tilde{K}_{i}^{n} F_{i}^{(n)}, & T_{i}^{-1}\left(E_{i}^{(n)}\right)=(-1)^{n} q_{i}^{n(n-1)} F_{i}^{(n)} \tilde{K}_{i}^{-n}, \\
T_{i}\left(F_{i}^{(n)}\right)=(-1)^{n} q_{i}^{-n(n-1)} E_{i}^{(n)} \tilde{K}_{i}^{-n}, & T_{i}^{-1}\left(F_{i}^{(n)}\right)=(-1)^{n} \pi_{i}^{n} q_{i}^{-n(n-1)} \tilde{J}_{i}^{n} \tilde{K}_{i}^{n} E_{i}^{(n)}, \\
\left.T_{i}\left(E_{j}^{(n)}\right)=\pi_{i}^{\left({ }^{n}{ }^{2} j\right.}\right) \tilde{J}_{i}^{n p(j)} e_{i, j, n,-n a_{i j},}, & T_{i}^{-1}\left(E_{j}^{(n)}\right)=\pi_{i}^{\left({ }^{n} a_{i j}\right)} \tilde{J}_{i}^{n p(j)} e_{i, j, n,-n a_{i j},}^{\prime}, \\
T_{i}\left(F_{j}^{(n)}\right)=\tilde{J}_{i}^{n p(j)} f_{i, j, n,-n a_{i j},}, & T_{i}^{-1}\left(F_{j}^{(n)}\right)=\tilde{J}_{i}^{n p(j)} f_{i, j, n,-n a_{i j}}^{\prime}, \\
T_{i}\left(K_{\mu}\right)=K_{s_{i}(\mu)}, & T_{i}^{-1}\left(K_{\mu}\right)=K_{s_{i}(\mu),}, \\
T_{i}\left(J_{\mu}\right)=J_{s_{i}(\mu)}, & T_{i}^{-1}\left(J_{\mu}\right)=J_{s_{i}(\mu)},
\end{array}
$$

where the elements $e_{i, j, n,-n a_{i j}}, e_{i, j, n,-n a_{i j}}^{\prime}, f_{i, j, n,-n a_{i j}}, f_{i, j, n,-n a_{i j}}^{\prime}$ are defined in (2.31)(2.34).

Remark 3.3.2. Observe that the above formulas imply that the braiding operators are even automorphisms of $\mathbf{U}$ (in the nontrivial cases, this follows from the fact that $\left\langle\alpha_{i}^{\vee}, \alpha_{j}\right\rangle$ is even when $\left.i \in I_{\overline{1}}\right)$. In contrast, the definition of $T_{i}$ as a map $M_{\lambda} \longrightarrow M_{s_{i}(\lambda)}$ implies that its parity is $\left\langle\alpha_{i}^{\vee}, \lambda\right\rangle$ as noted in (3.4).

One may verify directly on the generators that

$$
T_{i} \sigma=\sigma T_{i}^{-1} \text {. }
$$

Furthermore, by inspection of the images of the generators in Theorem 3.3.1, we see that $T_{i}^{ \pm 1}$ preserve the integral form of $\mathbf{U}$. In particular, this implies the following corollary.

Corollary 3.3.3. The automorphisms $T_{i}$ and $T_{i}^{-1}$ of $\mathbf{U}$ restrict to automorphisms of ${ }_{\mathcal{A}} \mathbf{U}$.

Remark 3.3.4. In CFLW, C], a modified form $\dot{\mathbf{U}}$ of $\mathbf{U}$ was defined à la Lusztig; to wit, one adds weight-space projections $1_{\lambda}$ for each $\lambda \in P$ to $\mathbf{U}$ to obtain an algebra $\dot{\mathbf{U}}$ on symbols $u 1_{\lambda}$, where $u \in \mathbf{U}$ and $\lambda \in P$, subject to some natural relations. We note that, just as in $[\mathrm{L}, \S 41.1]$, this modified form admits braiding operators $T_{i}^{ \pm 1}$ $(i \in I)$ satisfying $T_{i}^{ \pm 1}\left(u 1_{\lambda}\right)=T_{i}^{ \pm 1}(u) 1_{s_{i}(\lambda)}$, which restrict to automorphisms of the integral form of $\mathbf{U}$.

3.4. Braiding operators and comultiplication. Let $M, N \in \mathcal{O}_{\text {int }}^{i}$. As usual, we regard $M \otimes N$ as a $\mathbf{U}$-module via $\Delta$, and note that $M \otimes N \in \mathcal{O}_{\text {int }}^{i}$. If $x \in M_{t}$ and $y \in N_{s}$, then $x \otimes y \in(M \otimes N)_{t+s}$ and

$$
\begin{gathered}
\Delta\left(E_{i}\right)(x \otimes y)=E_{i} x \otimes y+\pi_{i}^{p(x)}\left(\pi_{i} q_{i}\right)^{t} x \otimes E_{i} y, \\
\Delta\left(F_{i}\right)(x \otimes y)=q_{i}^{-s} F_{i} x \otimes y+\pi_{i}^{p(x)} x \otimes F_{i} y .
\end{gathered}
$$


Define operators $L_{i}^{\prime}, L_{i}^{\prime \prime}: M \otimes N \longrightarrow M \otimes N$ by

$$
\begin{gathered}
L_{i}^{\prime}(x \otimes y)=\sum_{n \geq 0}(-1)^{n} \pi_{i}^{n}\left(\pi_{i} q_{i}\right)^{\left(\begin{array}{c}
n \\
2
\end{array}\right)}\left(\pi_{i} q_{i}-q_{i}^{-1}\right)^{n}[n]_{i}^{!} F_{i}^{(n)} x \otimes E_{i}^{(n)} y, \\
L_{i}^{\prime \prime}(x \otimes y)=\sum_{n \geq 0}(-1)^{n} \pi_{i}^{n} q_{i}^{-\left(\begin{array}{c}
n \\
2
\end{array}\right)}\left(\pi_{i} q_{i}-q_{i}^{-1}\right)^{n}[n]_{i}^{!} F_{i}^{(n)} x \otimes E_{i}^{(n)} y .
\end{gathered}
$$

These operators are the precisely the operators $\bar{\Theta}$ and $\Theta$, respectively, for the algebra $\mathbf{U}(i)$ defined in CHW1, §3.1]. In particular, we have the properties

$$
\begin{gathered}
L_{i}^{\prime} L_{i}^{\prime \prime}=L_{i}^{\prime \prime} L_{i}^{\prime}=1: M \otimes N \longrightarrow M \otimes N, \\
L_{i}^{\prime} \Delta(u)=\bar{\Delta}(u) L_{i}^{\prime}
\end{gathered}
$$

We are therefore justified to introduce the new notations

$$
L_{i}=L_{i}^{\prime} \quad \text { and } \quad L_{i}^{-1}=L_{i}^{\prime \prime} .
$$

We also note the following lemma follows from (3.11).

Lemma 3.4.1. Let $x \in M_{t}$ and $y \in M_{s}$. Then,

$$
\Delta\left(F_{i}\right) L_{i}^{-1}(x \otimes y)=L_{i}^{-1}\left(\left(\pi_{i} q_{i}\right)^{s} F_{i} x \otimes y+\pi_{i}^{p(x)} x \otimes F_{i} y\right),
$$

and

$$
\Delta\left(E_{i}\right) L_{i}^{-1}(x \otimes y)=L_{i}^{-1}\left(E_{i} x \otimes y+\pi_{i}^{p(x)} q_{i}^{-t} x \otimes E_{i} y\right) .
$$

We will now relate the action of $T_{i}, T_{i}^{-1}$ on a tensor product of modules to their actions on each tensor factor. Recall from (3.4) that the maps $T_{i}$ are not generally homogeneous with respect to the $\mathbb{Z} / 2 \mathbb{Z}$-grading. Consequently, when applying $T_{i}$ to the second tensor factor, we incur a $\pi$ factor depending on the weight of the second tensor factor and the parity of the first tensor factor. Therefore, we define the maps $T_{i} \otimes T_{i}$ and $T_{i}^{-1} \otimes T_{i}^{-1}$ to be the $\mathbb{Q}^{\pi}(q)$-linear maps defined by

$$
\begin{gathered}
\left(T_{i} \otimes T_{i}\right)(m \otimes n)=\pi_{i}^{s p(m)} T_{i}(m) \otimes T_{i}(n), \\
\left(T_{i}^{-1} \otimes T_{i}^{-1}\right)(m \otimes n)=\pi_{i}^{s p(m)} T_{i}^{-1}(m) \otimes T_{i}^{-1}(n),
\end{gathered}
$$

for $m \otimes n \in M_{t} \otimes N_{s}$. Note that $\left(T_{i} \otimes T_{i}\right)^{-1}(m \otimes n)=\pi_{i}^{s t} T_{i}^{-1} \otimes T_{i}^{-1}(m \otimes n)$. Then $L_{i}$ intertwines with the operators $T_{i}$ as follows.

Lemma 3.4.2. Let $M, N \in \mathcal{O}_{\text {int }}^{i}$. Then, for any $z \in M \otimes N$,

$$
\left(T_{i}^{-1} \otimes T_{i}^{-1}\right) \circ T_{i} \circ L_{i}^{-1}(z)=z .
$$

Proof. First, we shall prove that (3.12) holds for $z=x \otimes y \in M \otimes N$, then it also holds for $z^{\prime}=\bar{\Delta}\left(F_{i}\right) z=\left(\pi_{i} q_{i}\right)^{s} F_{i} x \otimes y+x \otimes F_{i} y$. We may assume $x \in M_{t}$ and $y \in N_{s}$, and so by assumption

$$
T_{i} \circ L_{i}^{-1}(z)=\pi_{i}^{s t}\left(T_{i} \otimes T_{i}\right)(z) .
$$

By Lemmas 3.4.1 and 3.1.6(a),

$$
T_{i}\left(L_{i}^{-1}\left(z^{\prime}\right)\right)=T_{i}\left(\Delta\left(F_{i}\right) L_{i}^{-1}(z)\right)=-q_{i}^{t+s} \Delta\left(E_{i}\right) T_{i}\left(L_{i}^{-1}(z)\right) .
$$


On the other hand, we have

$$
\begin{aligned}
\left(T_{i} \otimes T_{i}\right)\left(z^{\prime}\right) & =\left(T_{i} \otimes T_{i}\right)\left(\left(\pi_{i} q_{i}\right)^{s} F_{i} x \otimes y+\pi_{i}^{p(x)} x \otimes F_{i} y\right) \\
& =\left(\pi_{i} q_{i}\right)^{s} \pi_{i}^{(1+p(x)) s} T_{i}\left(F_{i} x\right) \otimes T_{i}(y)+\pi_{i}^{(s+1) p(x)} T_{i}(x) \otimes T_{i}\left(F_{i} y\right) \\
& =\pi_{i}^{s p(x)}\left(\left(-q_{i}^{s+t} E_{i} T_{i}(x) \otimes T_{i}(y)\right)+\pi_{i}^{p(x)} T_{i}(x) \otimes\left(-q_{i}^{s} E_{i} T_{i}(y)\right)\right. \\
& \stackrel{(\mathrm{a})}{=}-q_{i}^{s+t} \pi_{i}^{s p(x)}\left(E_{i} \otimes 1+q_{i}^{-t} \pi_{i}^{t} 1 \otimes E_{i}\right)\left(T_{i}(x) \otimes T_{i}(y)\right) \\
& \stackrel{(\mathrm{b})}{=}-q_{i}^{s+t} \Delta\left(E_{i}\right)\left(T_{i} \otimes T_{i}\right)(x \otimes y) .
\end{aligned}
$$

We note that the equality (a) follows from $p\left(T_{i}(x)\right)=t p(i)+p(x)$, whereas (b) follows since $T_{i}(x) \in M_{-t}$. Then applying the induction hypothesis, we have shown that $T_{i}\left(L_{i}^{-1}\left(z^{\prime}\right)\right)=\pi^{s t}\left(T_{i} \otimes T_{i}\right)\left(z^{\prime}\right)$; since $\pi_{i}^{s t}=\pi_{i}^{(s-2) t}=\pi_{i}^{s(t-2)}$, we have

$$
\left(T_{i}^{-1} \otimes T_{i}^{-1}\right) \circ T_{i} \circ L_{i}^{-1}\left(z^{\prime}\right)=z^{\prime},
$$

and thus the claim is proved.

Following [L, Proposition 5.3.4], define $Z_{\ell} \subset M \otimes N, \ell \geq 0$, to be the subspace spanned by vectors of the form $x \otimes F^{(q)} y$ with $E_{i} y=0$. Then $M \otimes N=\sum_{\ell \geq 0} Z_{\ell}$. We prove (a) by induction on $\ell$. In particular, we will show:

(1) The identity (3.12) holds for $z \in Z_{0}$, and

(2) If the identity (3.12) holds for $z \in Z_{\ell}$, then it holds for $z \in Z_{\ell+1}$.

The proof of (2) is exactly as in $\mathrm{L}$. Indeed, assume (3.12) holds for $z \in Z_{\ell}$ and write $z=x \otimes F_{i}^{(\ell)} y$ with $E_{i} y=0$. Then, by the discussion above, (3.12) also holds for $z^{\prime}=\left(\pi_{i} q_{i}\right)^{s} F_{i} x \otimes F_{i}^{(\ell)} y+[\ell+1]_{i} x \otimes F_{i}^{(\ell+1)} y$. Since, by assumption (3.12) holds for $z^{\prime \prime}=\left(\pi_{i} q_{i}\right)^{s} F_{i} x \otimes F_{i}^{(\ell)} y$, it holds for $z^{\prime \prime \prime}=[\ell+1]_{i} x \otimes F_{i}^{(\ell+1)} y$ as well. Since $[\ell+1]_{i} \neq 0,(2)$ is proved.

We now prove the base case (1). To this end, assume $z=x \otimes y \in M_{m} \otimes N_{n}$ with $E_{i} y=0$. Then by the definition of $L_{i}, L_{i}(z)=z$ and so it remains to show that $T_{i}(z)=\pi^{m n}\left(T_{i} \otimes T_{i}\right) z$. By Lemma 3.1.5, we deduce that

$$
\begin{aligned}
T_{i}(z) & \left.=(-1)^{m+n} \pi_{i}^{(m+n+1}\right) q_{i}^{-(m+n)} \overline{T_{i}^{-1}(\bar{z})} \\
& =(-1)^{m+n}\left(\pi_{i} q_{i}\right)^{-(m+n)} \sum_{-a+b-c=m+n}(-1)^{b} \pi_{i}^{c+b+a(m+n)} q_{i}^{-a c+b} E_{i}^{(a)} F_{i}^{(b)} E_{i}^{(c)} z .
\end{aligned}
$$

Let $\star=(-1)^{m+n}\left(\pi_{i} q_{i}\right)^{m+n} T_{i}(x \otimes y)$. Using (3.1) and (2.29), we compute

$$
\begin{gathered}
\star=\sum_{-a^{\prime}-a^{\prime \prime}+b^{\prime}+b^{\prime \prime}-c=m+n}(-1)^{b^{\prime}+b^{\prime \prime}} \pi_{i}^{c+b^{\prime}+b^{\prime \prime}+a^{\prime}(m+n)+a^{\prime \prime}(m+n)} q_{i}^{-a^{\prime} c-a^{\prime \prime} c+b^{\prime}+b^{\prime \prime}} \\
\times q_{i}^{a^{\prime} a^{\prime \prime}}\left(E_{i}^{\left(a^{\prime}\right)}\left(\tilde{J}_{i} \tilde{K}_{i}\right)^{a^{\prime \prime}} \otimes E_{i}^{\left(a^{\prime \prime}\right)}\right)\left(\pi_{i} q_{i}\right)^{-b^{\prime} b^{\prime \prime}}\left(F_{i}^{\left(b^{\prime}\right)} \otimes \tilde{K}_{i}^{-b^{\prime}} F_{i}^{\left(a^{\prime \prime}\right)}\right)\left(E_{i}^{(c)} x \otimes y\right) \\
=\sum_{-a^{\prime}-a^{\prime \prime}+b^{\prime}+b^{\prime \prime}-c=m+n}(-1)^{b^{\prime}+b^{\prime \prime}} q_{i}^{-a^{\prime} c-a^{\prime \prime} c+b^{\prime}+b^{\prime \prime}+a^{\prime} a^{\prime \prime}+b^{\prime} b^{\prime \prime}-b^{\prime} n+a^{\prime \prime}\left(m+2 c-2 b^{\prime}\right)} \\
\quad \times \pi_{i}^{c+b^{\prime}+b^{\prime \prime}+a^{\prime}(m+n)+a^{\prime \prime}(m+n)+b^{\prime} b^{\prime \prime}+b^{\prime \prime} c+\left(a^{\prime \prime}+b^{\prime \prime}\right) p(x)+a^{\prime \prime} b^{\prime}+a^{\prime \prime} c+a^{\prime \prime} m} \\
\left.\quad \times \pi_{i}^{a^{\prime \prime} b^{\prime \prime}+\left(a_{2}^{\prime \prime}+1\right.}\right)\left[\begin{array}{c}
a^{\prime \prime}-b^{\prime \prime}+n \\
a^{\prime \prime}
\end{array}\right]_{i}\left(E_{i}^{\left(a^{\prime}\right)} F_{i}^{\left(b^{\prime}\right)} E_{i}^{(c)} x \otimes F_{i}^{\left(b^{\prime \prime}-a^{\prime \prime}\right)} y\right)
\end{gathered}
$$


Now, make the substitution $b^{\prime \prime}=a^{\prime \prime}+g$. We note that, since $E_{i} y=0$ and $y \in N_{n}$, $F_{i}^{(g)} y=0$ for $g>n$ and hence the sum above is nonzero only when $g \leq n$. Then we may rewrite the above to obtain

$$
\begin{aligned}
\star & =\sum_{-a^{\prime}+b^{\prime}-c=m+n-g}(-1)^{b^{\prime}+g} \pi_{i}^{c+b^{\prime}+a^{\prime}(m+n+g)+(m+n) g+g p(x)} q_{i}^{-a^{\prime} c+b^{\prime}+g+b^{\prime} g-b^{\prime} n} \\
& \left.\times\left(\sum_{a^{\prime \prime} \geq 0}(-1)^{a^{\prime \prime}} \pi_{i}^{a^{\prime \prime}(n+g)+\left(a^{\prime \prime}+1\right.}\right) q_{i}^{a^{\prime \prime}(1+(n-g))}\left[\begin{array}{c}
n-g \\
a^{\prime \prime}
\end{array}\right]_{i}\right) E_{i}^{\left(a^{\prime}\right)} F_{i}^{\left(b^{\prime}\right)} E_{i}^{(c)} x \otimes F_{i}^{(g)} y
\end{aligned}
$$

Now using the image of the identity (2.9) under -, it follows that the sum over $a^{\prime \prime}$ is zero unless $n-g=0$. Now multiplying $\star$ by $(-1)^{m+n}\left(\pi_{i} q_{i}\right)^{-m-n}$, we have

$$
\begin{aligned}
& T_{i}(x \otimes y)=\pi_{i}^{m n+n p(x)}(-1)^{m}\left(\pi_{i} q_{i}\right)^{m} \pi_{i}^{\left(\begin{array}{c}
m \\
2
\end{array}\right)} \\
& \times\left(\sum_{-a^{\prime}+b^{\prime}-c=m}(-1)^{b^{\prime}} \pi_{i}^{c+b^{\prime}+a^{\prime} m+\left(\begin{array}{c}
m \\
2
\end{array}\right)} q_{i}^{-a^{\prime} c+b^{\prime}} E_{i}^{\left(a^{\prime}\right)} F_{i}^{\left(b^{\prime}\right)} E_{i}^{(c)} x\right) \otimes F_{i}^{(n)} y \\
& =\pi_{i}^{m n+n p(x)} T_{i}(x) \otimes T_{i}(y)=\pi_{i}^{m n}\left(T_{i} \otimes T_{i}\right)(x \otimes y) .
\end{aligned}
$$

This completes the proof.

Corollary 3.4.3. Let $M, N \in \mathcal{O}_{\text {int }}$ and $u \in \mathbf{U}$. Then as maps on $M \otimes N$,

$$
L_{i} \Delta(u) L_{i}^{-1}=\left(T_{i}^{-1} \otimes T_{i}^{-1}\right) \Delta\left(T_{i}(u)\right) .
$$

Proof. Let $z=x \otimes y \in M_{\lambda} \otimes N_{\mu}$. Inserting $L_{i}(z)$ into Lemma 3.4.2, we deduce that

Therefore, for $u \in \mathbf{U}$,

$$
\left(T_{i}^{-1} \otimes T_{i}^{-1}\right) T_{i}(z)=L_{i}(z)
$$

$$
\begin{aligned}
L_{i} \Delta(u) L_{i}^{-1}(z) & =L_{i}\left(u L_{i}^{-1}(z)\right) \\
& =L_{i}\left(u T_{i}^{-1} T_{i}\left(L_{i}^{-1}(z)\right)\right) \\
& =\pi_{i}^{\left\langle\alpha_{i}^{\vee}, \lambda\right\rangle\left\langle\alpha_{i}^{\vee}, \mu\right\rangle} L_{i}\left(u T_{i}^{-1}\left(T_{i} \otimes T_{i}\right)(z)\right) \\
& =\pi_{i}^{\left\langle\alpha_{i}^{\vee}, \lambda\right\rangle\left\langle\alpha_{i}^{\vee}, \mu\right\rangle}\left(T_{i}^{-1} \otimes T_{i}^{-1}\right) T_{i}\left(u T_{i}^{-1}\left(T_{i} \otimes T_{i}\right)(z)\right) \\
& =\pi_{i}^{\left\langle\alpha_{i}^{\vee}, \lambda\right\rangle\left\langle\alpha_{i}^{\vee}, \mu\right\rangle}\left(T_{i}^{-1} \otimes T_{i}^{-1}\right)\left(T_{i}(u)\left(T_{i} \otimes T_{i}\right)(z)\right) \\
& =\left(T_{i}^{-1} \otimes T_{i}^{-1}\right) \Delta\left(T_{i}(u)\right)(z) .
\end{aligned}
$$

This proves the corollary.

\section{BRAID GROUP ACTION AND THE INNER PRODUCT}

4.1. Algebras $\mathbf{U}_{J}^{0}$ and $\mathbf{U}_{J}^{+}$. Recall that the $\mathbb{Q}^{\pi}(q)$-algebra $\mathbf{U}^{0}$ has a basis

$$
\left\{J_{\mu} K_{\nu} \mid \mu, \nu \in P^{\vee}\right\} \text {. }
$$

Denote by $\mathbf{U}_{J}^{0}$ the $\mathbb{Q}^{\pi}(q)$-subalgebra of $\mathbf{U}^{0}$ generated by $J_{i}$ for $i \in I_{\overline{1}}$ (or equivalently, generated by $\tilde{J}_{i}$ for $\left.i \in I\right)$. Then clearly $\mathbf{U}_{J}^{0}$ is a free $\mathbb{Q}^{\pi}(q)$-module with basis $\left\{J_{\nu} \mid \nu \in \sum_{i \in I_{\overline{1}}} \mathbb{Z} \alpha_{i}^{\vee}\right\}$. Moreover, note that we can view $\mathbf{U}$ as an algebra over $\mathbf{U}_{J}^{0}$ which, by the triangular decomposition, is free as a $\mathbf{U}_{J}^{0}$-module. We note that the braid operators are $\mathbf{U}_{J}^{0}$-linear maps by Lemma 3.2.1 and Theorem 3.3.1 
Denote by $\mathbf{U}_{J}^{+}$the $\mathbb{Q}^{\pi}(q)$-subalgebra of $\mathbf{U}$ generated by $E_{i}, \tilde{J}_{i}(i \in I)$, or equivalently, generated by the subalgebras $\mathbf{U}^{+}$and $\mathbf{U}_{J}^{0}$. We can endow $\mathbf{U}_{J}^{+}$with a twisted bialgebra structure analogous to $\mathbf{f}$. We transport the maps ${ }_{i} r, r_{i}: \mathbf{f} \rightarrow \mathbf{f}$ as follows. Define $q$-derivations ${ }_{i} r$ and $r_{i}$ on $\mathbf{U}_{J}^{+}$by ${ }_{i} r\left(\tilde{J}_{\mu} x\right)=\tilde{J}_{\mu i} r(y)^{+}$and $r_{i}\left(\tilde{J}_{\nu} x\right)=\tilde{J}_{\nu} r_{i}(y)^{+}$ if $y \in \mathbf{f}$ satisfies $y^{+}=x \in \mathbf{U}^{+}$, and $\nu \in Q_{+}$. Next, define

$$
r: \mathbf{U}_{J}^{+} \longrightarrow \mathbf{U}_{J}^{+} \otimes \mathbf{U}_{J}^{+}
$$

by $r(x)=\sum y_{(1)}^{+} \otimes y_{(2)}^{+}$if $y \in \mathbf{f}$ satisfies $y^{+}=x$ and $r(y)=\sum y_{(1)} \otimes y_{(2)}$, and $r\left(\tilde{J}_{\nu}\right)=\tilde{J}_{\nu} \otimes \tilde{J}_{\nu}$ for all $\nu \in Q_{+}$. Then, $r$ is an algebra homomorphism with respect to the twisted multiplication (2.11). Moreover, recall the anti-automorphism $\sigma$ : $\mathbf{U} \rightarrow \mathbf{U}$ defined in (2.26). Then for $x \in \mathbf{U}_{J}^{+}$with $r(x)=\sum x_{1} \otimes x_{2}$, we have

$$
r(\sigma(x))=\sum \sigma\left(x_{2}\right) \otimes \sigma\left(x_{1}\right) .
$$

In particular, $r_{i} \circ \sigma=\sigma \circ{ }_{i} r$.

Finally, define a bilinear form $(\cdot, \cdot): \mathbf{U}_{J}^{+} \otimes \mathbf{U}_{J}^{+} \longrightarrow \mathbf{U}_{J}^{0}$ by

$$
\left(\tilde{J}_{\nu_{1}} x_{1}, \tilde{J}_{\nu_{2}} x_{2}\right)=\tilde{J}_{\nu_{1}+\nu_{2}}\left(y_{1}, y_{2}\right) \text { if } y_{1}^{+}=x_{1}, y_{2}^{+}=x_{2} \text {, and } \nu_{1}, \nu_{2} \in Q_{+} .
$$

We note that, from the definitions, analogues of (2.14) and (2.16) hold for this bilinear form.

4.2. The algebras $\mathbf{U}_{J}^{+}[i]$ and ${ }^{\sigma} \mathbf{U}_{J}^{+}[i]$. Fix $i \in I$, and for any $j \in I \backslash\{i\}$ set

$$
\begin{aligned}
e(i, j ; m) & =\sum_{r+s=m}(-1)^{r} \pi_{i}^{p(r ; i, j)}\left(\pi_{i} q_{i}\right)^{-r\left(a_{i j}+m-1\right)} E_{i}^{(r)} E_{j} E_{i}^{(s)} \in \mathbf{U}^{+} ; \\
e^{\prime}(i, j ; m) & =\sum_{r+s=m}(-1)^{r} \pi_{i}^{p(r ; i, j)}\left(\pi_{i} q_{i}\right)^{-r\left(a_{i j}+m-1\right)} E_{i}^{(s)} E_{j} E_{i}^{(r)} \in \mathbf{U}^{+} ;
\end{aligned}
$$

where $p(r ; i, j)=p(r, 1 ; i, j)=r p(i) p(j)+\left(\begin{array}{l}r \\ 2\end{array}\right) p(i)$. Then $e(i, j ; m)=e_{i, j ; 1, m}$ and $e^{\prime}(i, j ; m)=e_{i, j ; 1, m}^{\prime}$.

Let $\mathbf{U}_{J}^{+}[i]$ (resp. $\left.{ }^{\sigma} \mathbf{U}_{J}^{+}[i]\right)$ be the $\mathbf{U}_{J}^{0}$-subalgebra of $\mathbf{U}_{J}^{+}$generated by $e(i, j ; m)$ (resp. $\left.e^{\prime}(i, j ; m)\right)$ for $m \geq 0$ and $j \in I \backslash\{i\}$. Since $\sigma(e(i, j ; m))=e^{\prime}(i, j ; m)$, we have $\sigma\left(\mathbf{U}_{J}^{+}[i]\right)={ }^{\sigma} \mathbf{U}_{J}^{+}[i]$.

Lemma 4.2.1. (a) $\mathbf{U}_{J}^{+}=\sum_{t>0} E_{i}^{t} \mathbf{U}_{J}^{+}[i]=\sum_{t>0} \mathbf{U}_{J}^{+}[i] E_{i}^{t}$;

(b) $\mathbf{U}_{J}^{+}=\sum_{t \geq 0} \sigma \mathbf{U}_{J}^{+}[i] E_{i}^{t}=\sum_{t \geq 0} E_{i}^{t \sigma} \mathbf{U}_{J}^{+}[i]$.

Proof. Clearly (b) follows from (a) by applying $\sigma$. To prove (a), note that Lemma 2.6.1 provides the relation

$$
e(i, j ; m) E_{i}-q_{i}^{-a_{i j}-2 m} \pi_{i}^{m+n p(j)} E_{i} e(i, j ; m)=[m+1]_{i} e(i, j ; m+1) .
$$

Therefore, given any product $y_{1} \cdots y_{n}$ in which each factor is either $E_{i}$ or one of the $e(i, j ; m)$, we may use this relation to rewrite it either as a linear combination of products of the form $E_{i}^{t} y_{1}^{\prime} \cdots y_{k}^{\prime}$, where $y_{1}^{\prime}, \ldots, y_{k}^{\prime} \in \mathbf{U}_{J}^{+}[i]$, or as a linear combination of products of the form $y_{1}^{\prime \prime} \cdots y_{k}^{\prime \prime} E_{i}^{t}$, where $y_{1}^{\prime \prime}, \ldots, y_{k}^{\prime \prime} \in \mathbf{U}_{J}^{+}[i]$. Now, the result follows from the fact that $\mathbf{U}_{J}^{+}$is generated by $\mathbf{U}_{J}^{0}$, together with $E_{i}$ and $E_{j}=e(i, j ; 0)$ for $j \neq i$.

Lemma 4.2.2. Assume $i, j \in I, i \neq j$. For any $0 \leq m \leq-a_{i j}$,

(a) $T_{i}\left(e^{\prime}(i, j ; m)\right)=\pi_{i}^{\left(a_{i j}^{2}\right)} \pi_{i}^{(p(j)+1)\left(-a_{i j}-m\right)} \tilde{J}_{i}^{p(j)} e\left(i, j ;-a_{i j}-m\right)$;

(b) $T_{i}^{-1}(e(i, j ; m))=\pi_{i}^{\left(\begin{array}{c}a_{2 j} \\ 2\end{array}\right)} \pi_{i}^{(p(j)+1)\left(-a_{i j}-m\right)} \tilde{J}_{i}^{p(j)} e^{\prime}\left(i, j ;-a_{i j}-m\right)$. 
Proof. The statements are equivalent by Proposition 3.1.4. We prove (a) by downward induction on $m$, the initial case $m=-\left\langle i, j^{\prime}\right\rangle$ being Lemma 3.2.3(b). To this end, recall that by Lemma 2.6.1

$$
-F_{i} e(i, j ; m)+\pi_{i}^{m+p(j)} e(i, j ; m) F_{i}=\left[-n a_{i j}-m+1\right]_{i} \pi_{i}^{p(j)+1} \tilde{J}_{i}^{-1} e(i, j ; m-1) .
$$

Applying the anti-automorphism $\sigma$, we obtain the equation

$\pi_{i} \tilde{J}_{i}\left(-e^{\prime}(i, j ; m) F_{i}+\pi_{i}^{m+p(j)} F_{i} e^{\prime}(i, j ; m)\right)=\left[-n a_{i j}-m+1\right]_{i} \pi_{i}^{p(j)+1} e^{\prime}(i, j, m-1) \tilde{J}_{i}$.

Applying $T_{i}$ to both sides, and applying the induction hypothesis together with Lemma 2.6.1 and Theorem 3.3.1, we have

$$
\begin{aligned}
{\left[-n a_{i j}-\right.} & m+1]_{i} \pi_{i}^{p(j)+1} T_{i}\left(e^{\prime}(i, j ; m-1)\right) \tilde{J}_{i}^{-1} \\
= & \pi_{i}^{\left(\begin{array}{c}
a_{i j} \\
2
\end{array}\right)} \pi_{i}^{(p(j)+1)\left(-a_{i j}-m\right)} \tilde{J}_{i}^{p(j)}\left(e\left(i, j ;-a_{i j}-m\right) E_{i}\right. \\
& \left.\quad-q_{i}^{-a_{i j}-2 m} \pi_{i}^{m+p(j)} E_{i} e\left(i, j ;-a_{i j}-m\right)\right) \tilde{J}_{i}^{-1} \\
= & \pi_{i}^{\left(\begin{array}{c}
a_{i j} \\
2
\end{array}\right)} \pi_{i}^{(p(j)+1)\left(-a_{i j}-m\right)} \tilde{J}_{i}^{p(j)}\left[-n a_{i j}-m+1\right] e\left(i, j ;-a_{i j}-m+1\right) \tilde{J}_{i}^{-1} .
\end{aligned}
$$

Therefore, (a) follows.

The next lemma is a consequence of Lemma 4.2 .2

Lemma 4.2.3. The braiding operator $T_{i}^{-1}$ defines an isomorphism of $\mathbf{U}_{J}^{+}[i]$ onto ${ }^{\sigma} \mathbf{U}_{J}^{+}[i]$ with $T_{i}$ being the inverse isomorphism.

Lemma 4.2.4. Assume that $x \in \mathbf{U}_{J}^{+}$satisfies $T_{i}^{-1}(x) \in \mathbf{U}_{J}^{+}$. Then ${ }_{i} r(x)=0$.

Proof. By Proposition 2.4.4, we have for homogeneous $x \in \mathbf{U}_{J}^{+}$,

$$
x F_{i}-\pi_{i}^{p(x)} F_{i} x=\frac{\pi_{i}^{p(x)-p(i)} \tilde{J}_{i} \tilde{K}_{i}{ }_{i} r(x)-r_{i}(x) \tilde{K}_{-i}}{\pi_{i} q_{i}-q_{i}^{-1}}
$$

Using Lemma 4.2.1, we may write

$$
\frac{{ }_{i} r(x)}{\pi_{i} q_{i}-q_{i}^{-1}}=\sum_{t \geq 0} E_{i}^{(t)} y_{t}
$$

and

$$
\frac{r_{i}(x)}{\pi_{i} q_{i}-q_{i}^{-1}}=\sum_{t \geq 0} E_{i}^{(t)} z_{t}
$$

where $y_{t}, z_{t} \in \mathbf{U}_{J}^{+}[i]$ are homogeneous. Using Lemma 4.2.3, we have

$$
T_{i}^{-1}\left(y_{t}\right), T_{i}^{-1}\left(z_{t}\right) \in \mathbf{U}_{J}^{+}
$$

for all $t \geq 0$. Therefore, applying $T_{i}^{-1}$ to (4.3), be obtain

$$
\begin{aligned}
& -\pi_{i} \tilde{J}_{i}\left(T_{i}^{-1}(x) \tilde{K}_{i} E_{i}-\tilde{K}_{i} E_{i} T_{i}^{-1}(x)\right) \\
& =\sum_{t \geq 0}(-1)^{t} q_{i}^{t(t-1)} F_{i}^{(t)} \tilde{K}_{-t i}\left(\pi_{i}^{p(x)-p(i)} \tilde{J}_{i} \tilde{K}_{-i} T_{i}^{-1}\left(y_{t}\right)-T_{i}^{-1}\left(z_{t}\right) \tilde{K}_{i}\right) .
\end{aligned}
$$

By assumption, the left-hand side of (4.4) is in $\tilde{K}_{i} \mathbf{U}_{J}^{+}$, hence so is the right-hand side. Using the triangular decomposition of $\mathbf{U}$, we deduce that $T_{i}^{-1}\left(y_{t}\right)=0$ for all $t \geq 0$, and $T_{i}^{-1}\left(z_{t}\right)=0$ for all $t>0$. As $T_{i}^{-1}$ is an automorphism of $\mathbf{U}$, we deduce that $y_{t}=0$ for all $t \geq 0$ proving the claim (note, however, that we may have $z_{0} \neq 0$ ). 
Lemma 4.2.5. Let $x_{t} \in \mathbf{U}_{J}^{+}, t \geq 1$ belong to $\operatorname{ker}\left({ }_{i} r\right)$, where only finitely many are nonzero. Assume that $\sum_{t \geq 0} E_{i}^{(t)} x_{t}=0$ or $\sum_{t \geq 0} x_{t} E_{i}^{(t)}=0$. Then, $x_{t}=0$ for all $t$.

Proof. Assume $x_{t}=0$ for $t>N$. We prove the proposition by induction on $N$. If $N=0$, then the lemma is trivially true. Assume $N>0$. Then, using the fact that $x_{t} \in \operatorname{ker}\left({ }_{i} r\right)$, we have

$$
\begin{gathered}
0={ }_{i} r^{N}\left(\sum_{t \geq 0} E_{i}^{(t)} x_{t}\right)=q_{i}^{\left(\begin{array}{c}
N \\
2
\end{array}\right)} x_{N}, \text { or } \\
0={ }_{i} r^{N}\left(\sum_{t \geq 0} x_{t} E_{i}^{(t)}\right)=q_{i}^{N\left(\alpha_{i},\left|x_{N}\right|\right)+\left(\begin{array}{c}
N \\
2
\end{array}\right)} x_{N} .
\end{gathered}
$$

In particular, $x_{N}=0$ and induction applies.

Proposition 4.2.6. (a) The following three subspaces coincide:

$$
\mathbf{U}_{J}^{+}[i]=\left\{x \in \mathbf{U}_{J}^{+} \mid T_{i}^{-1}(x) \in \mathbf{U}_{J}^{+}\right\}=\left\{x \in \mathbf{U}_{J}^{+} \mid{ }_{i} r(x)=0\right\} .
$$

(b) The following three subspaces coincide:

$$
{ }^{\sigma} \mathbf{U}_{J}^{+}[i]=\left\{x \in \mathbf{U}_{J}^{+} \mid T_{i}(x) \in \mathbf{U}_{J}^{+}\right\}=\left\{x \in \mathbf{U}_{J}^{+} \mid r_{i}(x)=0\right\} .
$$

Proof. We obtain (b) from (a) by applying $\sigma$ using (3.7) and (4.1). To prove (a), note that by Lemmas 4.2 .3 and 4.2.4, we have

$$
\mathbf{U}_{J}^{+}[i] \subseteq\left\{x \in \mathbf{U}_{J}^{+} \mid T_{i}^{-1}(x) \in \mathbf{U}_{J}^{+}\right\} \subseteq\left\{\left.x \in \mathbf{U}_{J}^{+}\right|_{i} r(x)=0\right\} .
$$

Now, assume that $x \in \mathbf{U}_{J}^{+}$satisfies ${ }_{i} r(x)=0$. By Lemma 4.2.1, we may write $x=\sum_{t \geq 0} E_{i}^{(t)} x_{t}$ where the $x_{t}$ belong to the kernel of ${ }_{i} r$. Then, the sum

$$
0=\left(x_{0}-x\right)+\sum_{t \geq 1} E_{i}^{(t)} x_{t}
$$

satisfies the conditions of Lemma 4.2.5. In particular, $x-x_{0}=0$, so $x \in \mathbf{U}_{J}^{+}[i]$. This completes the proof.

Combining Lemma 4.2.5 and Proposition 4.2.6 yields the following refinement of Lemma 4.2.1.

Corollary 4.2.7. The following $\mathbb{Q}^{\pi}(q)$-module decompositions hold.

(a) $\mathbf{U}_{J}^{+}=\bigoplus_{t \geq 0} E_{i}^{t} \mathbf{U}_{J}^{+}[i]=\bigoplus_{t \geq 0} \mathbf{U}_{J}^{+}[i] E_{i}^{t}$, and in particular

$$
\mathbf{U}_{J}^{+}=E_{i} \mathbf{U}_{J}^{+} \oplus \mathbf{U}_{J}^{+}[i]=\mathbf{U}_{J}^{+} E_{i} \oplus \mathbf{U}_{J}^{+}[i] .
$$

(b) $\mathbf{U}_{J}^{+}=\bigoplus_{t \geq 0}{ }^{\sigma} \mathbf{U}_{J}^{+}[i] E_{i}^{t}=\bigoplus_{t \geq 0} E_{i}^{t \sigma} \mathbf{U}_{J}^{+}[i]$, and in particular

$$
\mathbf{U}_{J}^{+}=\mathbf{U}_{J}^{+} E_{i} \oplus{ }^{\sigma} \mathbf{U}_{J}^{+}[i]=E_{i} \mathbf{U}_{J}^{+} \oplus{ }^{\sigma} \mathbf{U}_{J}^{+}[i]
$$

Recall the map $r: \mathbf{U}_{J}^{+} \rightarrow \mathbf{U}_{J}^{+} \otimes \mathbf{U}_{J}^{+}$defined in SS4.1.

Lemma 4.2.8. Let $P(i, j ; m ; t)=\prod_{h=0}^{m-t-1}\left(1-\pi_{i}^{h+1-m} q_{i}^{2 h+2-2 m-2 a_{i j}}\right)$. Then we have the following identities.

(a) $r(e(i, j ; m))=1 \otimes e(i, j ; m)+\sum_{t=0}^{m}\left(\pi_{i} q_{i}\right)^{t(m-t)} P(i, j ; m ; t) e(i, j ; t) \otimes E_{i}^{(m-t)}$.

(b) $r\left(e^{\prime}(i, j ; m)\right)=e^{\prime}(i, j ; m) \otimes 1+\sum_{t=0}^{m}\left(\pi_{i} q_{i}\right)^{t(m-t)} P(i, j ; m ; t) E_{i}^{(m-t)} \otimes e(i, j ; t)$. 
Proof. Using the fact that $r$ is an algebra homomorphism along with (2.12), we have

$$
\begin{aligned}
r(e(i, j ; m))= & (-1)^{r^{\prime}+r^{\prime \prime}} \pi_{i}^{p\left(r^{\prime}+r^{\prime \prime} ; i, j\right)}\left(\pi_{i} q_{i}\right)^{-\left(r^{\prime}+r^{\prime \prime}\right)\left(a_{i j}+m-1\right)-r^{\prime} r^{\prime \prime}-s^{\prime} s^{\prime \prime}} \\
& \times\left(E_{i}^{\left(r^{\prime}\right)} \otimes E_{i}^{\left(r^{\prime \prime}\right)}\right)\left(E_{j} \otimes 1+1 \otimes E_{j}\right)\left(E_{i}^{\left(s^{\prime}\right)} \otimes E_{i}^{\left(s^{\prime \prime}\right)}\right) \\
= & \sum(-1)^{r^{\prime}+r^{\prime \prime}} \pi_{i}^{p\left(r^{\prime}+r^{\prime \prime} ; i, j\right)+r^{\prime \prime} p(j)+r^{\prime \prime} s^{\prime}}\left(\pi_{i} q_{i}\right)^{-\left(r^{\prime}+r^{\prime \prime}\right)\left(a_{i j}+m-1\right)-r^{\prime} r^{\prime \prime}-s^{\prime} s^{\prime \prime}} \\
& \times q_{i}^{-r^{\prime \prime} a_{i j}-2 r^{\prime \prime} s^{\prime}}\left[\begin{array}{c}
r^{\prime \prime}+s^{\prime \prime} \\
r^{\prime \prime}
\end{array}\right]_{i} E_{i}^{\left(r^{\prime}\right)} E_{j} E_{i}^{\left(s^{\prime}\right)} \otimes E_{i}^{\left(r^{\prime \prime}+s^{\prime \prime}\right)} \\
+ & \sum(-1)^{r^{\prime}+r^{\prime \prime}} \pi_{i}^{p\left(r^{\prime}+r^{\prime \prime} ; i, j\right)+s^{\prime} p(j)+r^{\prime \prime} s^{\prime}}\left(\pi_{i} q_{i}\right)^{-\left(r^{\prime}+r^{\prime \prime}\right)\left(a_{i j}+m-1\right)-r^{\prime} r^{\prime \prime}-s^{\prime} s^{\prime \prime}} \\
& \times q_{i}^{-s^{\prime} a_{i j}-2 r^{\prime \prime} s^{\prime}}\left[\begin{array}{c}
r^{\prime}+s^{\prime} \\
r^{\prime}
\end{array}\right]_{i} E_{i}^{\left(r^{\prime}+s^{\prime}\right)} \otimes E_{i}^{\left(r^{\prime \prime}\right)} E_{j} E_{i}^{\left(s^{\prime \prime}\right)}
\end{aligned}
$$

where the sums are all over $r^{\prime}+r^{\prime \prime}+s^{\prime}+s^{\prime \prime}=m$.

Consider the sum (d). We note that the power of $\pi_{i}$ in (d) is

$$
p\left(r^{\prime}+r^{\prime \prime} ; i, j\right)+s^{\prime} p(j)+r^{\prime \prime} s^{\prime}=\left(r^{\prime}+s^{\prime}+r^{\prime \prime}\right) p(j)+\left(\begin{array}{c}
r^{\prime} \\
2
\end{array}\right)+\left(\begin{array}{c}
r^{\prime \prime} \\
2
\end{array}\right)+r^{\prime \prime}\left(s^{\prime}+r^{\prime}\right) \text {. }
$$

Writing $r^{\prime}+s^{\prime}=t$ and $r^{\prime \prime}+s^{\prime \prime}=m-t$, we have

$$
p\left(r^{\prime}+r^{\prime \prime} ; i, j\right)+s^{\prime} p(j)+r^{\prime \prime} s^{\prime}=p\left(r^{\prime \prime} ; i, j\right)+\left(\begin{array}{l}
r^{\prime} \\
2
\end{array}\right)+t p(j)+t r^{\prime \prime} .
$$

Similarly, since $s^{\prime} a_{i j}-2 r^{\prime \prime} s^{\prime} \in 2 \mathbb{Z}$, the power of $\pi_{i} q_{i}$ in (d) is $\varnothing$, where

$$
\begin{aligned}
\odot & =-\left(r^{\prime}+r^{\prime \prime}\right)\left(a_{i j}+m-1\right)-r^{\prime} r^{\prime \prime}-s^{\prime} s^{\prime \prime}-s^{\prime} a_{i j}-2 r^{\prime \prime} s^{\prime} \\
& =-r^{\prime \prime}\left(a_{i j}+m-1\right)-\left(r^{\prime}+s^{\prime}\right)\left(r^{\prime \prime}+a_{i j}\right)-r^{\prime} m+r^{\prime}-s^{\prime}\left(r^{\prime \prime}+s^{\prime \prime}\right) \\
& \left.=-r^{\prime \prime}\left(a_{i j}+m-1\right)-t\left(r^{\prime \prime}+a_{i j}\right)\right)-r^{\prime} m+r^{\prime}-s^{\prime}(m-t) \\
& =-r^{\prime \prime}\left(a_{i j}+m+t-1\right)-t a_{i j}-\left(s^{\prime}+r^{\prime}\right) m+s^{\prime} t+r^{\prime} \\
& =-r^{\prime \prime}\left(a_{i j}+m+t-1\right)-t\left(a_{i j}+m\right)+s^{\prime}(t-1)+s^{\prime}+r^{\prime} \\
& =-r^{\prime \prime}\left(a_{i j}+m+t-1\right)-t\left(a_{i j}+m-1\right)+s^{\prime}(t-1) \\
& =-r^{\prime \prime}\left(a_{i j}+m+t-1\right)-t\left(a_{i j}+m-1\right)+\left(t-r^{\prime}\right)(t-1) \\
& =-r^{\prime \prime}\left(a_{i j}+m+t-1\right)-t\left(a_{i j}+m-1\right)+2\left(\begin{array}{c}
t \\
2
\end{array}\right)-r^{\prime}(t-1)
\end{aligned}
$$

Therefore, we can rewrite (d) as

$$
\begin{aligned}
& \sum_{t=0}^{m} \sum_{r^{\prime \prime}+s^{\prime \prime}=m-t}(-1)^{r^{\prime \prime}} \pi_{i}^{p\left(r^{\prime \prime} ; i, j\right)+t p(j)+t r^{\prime \prime}}\left(\pi_{i} q_{i}\right)^{-r^{\prime \prime}\left(a_{i j}+m+t-1\right)-t\left(a_{i j}+m-1\right)+2\left(\begin{array}{c}
t \\
2
\end{array}\right)} \\
& \times \sum_{r^{\prime}+s^{\prime}=t}(-1)^{r^{\prime}} \pi_{i}^{\left(\begin{array}{c}
r^{\prime} \\
2
\end{array}\right)}\left(\pi_{i} q_{i}\right)^{-r^{\prime}(t-1)}\left[\begin{array}{c}
t \\
r^{\prime}
\end{array}\right]_{i} E_{i}^{(t)} \otimes E_{i}^{\left(r^{\prime \prime}\right)} E_{j} E_{i}^{\left(s^{\prime \prime}\right)}
\end{aligned}
$$

Applying the bar involution to (2.9), we conclude that the sum over $r^{\prime}+s^{\prime}$ is 0 unless $t=0$. Hence, (e) becomes

$$
1 \otimes \sum_{r^{\prime \prime}+s^{\prime \prime}=m}(-1)^{r^{\prime \prime}} \pi_{i}^{p\left(r^{\prime \prime} ; i, j\right)}\left(\pi_{i} q_{i}\right)^{-r^{\prime \prime}\left(a_{i j}+m-1\right)} E_{i}^{\left(r^{\prime \prime}\right)} E_{j} E_{i}^{\left(s^{\prime \prime}\right)}=1 \otimes e(i, j ; m) .
$$


Next, rewrite the sum corresponding to (c) in a similar manner to (d) to obtain

$$
\begin{aligned}
& \sum_{t=0}^{m} \sum_{r^{\prime \prime}+s^{\prime \prime}=m-t}(-1)^{r^{\prime \prime}} \pi_{i}^{\left(\begin{array}{c}
r^{\prime \prime} \\
2
\end{array}\right)+r^{\prime \prime} t}\left(\pi_{i} q_{i}\right)^{-r^{\prime \prime}\left(2 a_{i j}+m+t-1\right)}\left[\begin{array}{c}
m-t \\
r^{\prime \prime}
\end{array}\right]_{i} \\
& \times \sum_{r^{\prime}+s^{\prime}=t}(-1)^{r^{\prime}} \pi_{i}^{p\left(r^{\prime} ; i, j\right)}\left(\pi_{i} q_{i}\right)^{-r^{\prime}\left(a_{i j}+t-1\right)-(m-t) t} E_{i}^{\left(r^{\prime}\right)} E_{j} E_{i}^{\left(s^{\prime}\right)} \otimes E_{i}^{(m-t)} \\
& =\sum_{t=0}^{m}\left(\pi_{i} q_{i}\right)^{-(m-t) t} \sum_{r^{\prime \prime}+s^{\prime \prime}=m-t} \pi_{i}^{\left(r^{\prime \prime}\right)} q_{i}^{r^{\prime \prime}(m-t-1)}\left[\begin{array}{c}
m-t \\
r^{\prime \prime}
\end{array}\right]_{i}\left(-\pi_{i}^{1-m} q_{i}^{2-2 m-2 a_{i j} j}\right)^{r^{\prime \prime}} \\
& \times \sum_{r^{\prime}+s^{\prime}=t}(-1)^{r^{\prime}} \pi_{i}^{p\left(r^{\prime} ; i, j\right)}\left(\pi_{i} q_{i}\right)^{-r^{\prime}\left(a_{i j}+t-1\right)} E_{i}^{\left(r^{\prime}\right)} E_{j} E_{i}^{\left(s^{\prime}\right)} \otimes E_{i}^{(m-t)} \\
& =\sum_{t=0}^{m}\left(\pi_{i} q_{i}\right)^{-(m-t) t} \sum_{r^{\prime \prime}+s^{\prime \prime}=m-t} \pi_{i}^{\left(r^{\prime \prime}\right)} q_{i}^{r^{\prime \prime}(m-t-1)}\left[\begin{array}{c}
m-t \\
r^{\prime \prime}
\end{array}\right]_{i}\left(-\pi_{i}^{1-m} q_{i}^{2-2 m-2 a_{i j}}\right)^{r^{\prime \prime}} \\
& \times e(i, j ; t) \otimes E_{i}^{(m-t)}
\end{aligned}
$$

By evaluating the identity (2.7) at $z=-\pi_{i}^{1-m} q_{i}^{2-2 m-2 a_{i j}}$, we have

$$
\begin{aligned}
& \sum_{r^{\prime \prime}+s^{\prime \prime}=m-t} \pi_{i}^{\left(\begin{array}{c}
r^{\prime \prime} \\
2
\end{array}\right)} q_{i}^{r^{\prime \prime}(m-t-1)}\left[\begin{array}{c}
m-t \\
r^{\prime \prime}
\end{array}\right]_{i}\left(-\pi_{i}^{1-m} q_{i}^{2-2 m-2 a_{i j}}\right)^{r^{\prime \prime}} \\
= & \prod_{h=0}^{m-t-1}\left(1-\pi_{i}^{h+1-m} q_{i}^{2 h+2-2 m-2 a_{i j}}\right)
\end{aligned}
$$

which proves (a).

Finally, (b) follows from (a) since $r(\sigma(x))=(\sigma \otimes \sigma)^{t} r(x)$.

Lemma 4.2.9. Let $x \in \mathbf{U}_{J}^{+}[i]$, and let $y=T_{i}^{-1}(x) \in{ }^{\sigma} \mathbf{U}_{J}^{+}[i]$ (see Lemma 4.2.3). We have $r(x) \in \mathbf{U}_{J}^{+}[i] \otimes \mathbf{U}_{J}^{+}$and $r(y) \in{ }^{\sigma} \mathbf{U}_{J}^{+} \otimes \mathbf{U}_{J}^{+}[i]$.

Proof. Observe that if the lemma holds for $x_{1}$ and $x_{2}$ (resp. $y_{1}$ and $y_{2}$ ), then it holds for $x_{1} x_{2}$ (resp. $y_{1} y_{2}$ ) since, after twisting multiplication in $\mathbf{U}_{J}^{+} \otimes \mathbf{U}_{J}^{+}[i]$ (resp. $\left.{ }^{\sigma} \mathbf{U}_{J}^{+}[i] \otimes \mathbf{U}_{J}^{+}\right), r$ is multiplicative and $\mathbf{U}_{J}^{+}[i]$ (resp. ${ }^{\sigma} \mathbf{U}_{J}^{+}[i]$ ) is closed under multiplication. Therefore, it is enough to check the lemma for $x=e(i, j ; m)$ (resp. $\left.y=e^{\prime}(i, j ; m)\right)$. For these elements, the result follows from Lemma 4.2.8.

Let $x \in \mathbf{U}_{J}^{+}[i]$ and $y=T_{i}^{-1}(x) \in{ }^{\sigma} \mathbf{U}_{J}^{+}[i]$. Using the decomposition $\mathbf{U}_{J}^{+}=$ $\mathbf{U}_{J}^{+} E_{i} \oplus \mathbf{U}_{J}^{+}[i]$, let ${ }^{\prime} r(x) \in \mathbf{U}_{J}^{+}[i] \otimes \mathbf{U}_{J}^{+}[i]$ be the unique element such that

$$
r(x)-{ }^{\prime} r(x) \in \mathbf{U}_{J}^{+}[i] \otimes \mathbf{U}_{J}^{+} E_{i} .
$$

Using the decomposition $\mathbf{U}_{J}^{+}=E_{i} \mathbf{U}_{J}^{+} \oplus{ }^{\sigma} \mathbf{U}_{J}^{+}[i]$, let ${ }^{\prime \prime} r(y) \in{ }^{\sigma} \mathbf{U}_{J}^{+}[i] \otimes{ }^{\sigma} \mathbf{U}_{J}^{+}[i]$ be the unique element such that

$$
r(y)-{ }^{\prime \prime} r(y) \in E_{i} \mathbf{U}_{J}^{+} \otimes{ }^{\sigma} \mathbf{U}_{J}^{+}[i] .
$$

Lemma 4.2.10. We have $\left(T_{i}^{-1} \otimes T_{i}^{-1}\right)\left({ }^{\prime} r(x)\right)={ }^{\prime \prime} r\left(T_{i}^{-1}(x)\right)$.

Proof. Set $y=T_{i}^{-1}(x)$ as above. Let $\left\{u_{h}\right\}_{h \in H}$ be a homogeneous $\mathbf{U}_{J}^{0}$-basis for $\mathbf{U}_{J}^{+}[i]$. For each $h \in H$, set $v_{h}=T_{i}^{-1}\left(u_{h}\right)$, so $\left\{v_{h}\right\}_{h \in H}$ is a basis for ${ }^{\sigma} \mathbf{U}_{J}^{+}[i]$. Then, 
by Lemma 4.2.9, we may uniquely write

$$
\begin{aligned}
& r(x)=\sum_{n \geq 0 ; h, h^{\prime} \in H} c\left(n ; h, h^{\prime}\right) u_{h} \otimes u_{h^{\prime}} E_{i}^{(n)} \\
& r(y)=\sum_{n \geq 0 ; h, h^{\prime} \in H} d\left(n ; h, h^{\prime}\right) E_{i}^{(n)} v_{h} \otimes v_{h^{\prime}}
\end{aligned}
$$

where $c\left(n ; h, h^{\prime}\right), d\left(n ; h, h^{\prime}\right) \in \mathbf{U}_{J}^{0} \otimes \mathbf{U}_{J}^{0}$ are zero for all but finitely many indices. Note that we have

$$
\begin{aligned}
& { }^{\prime} r(x)=\sum_{h, h^{\prime} \in H} c\left(0 ; h, h^{\prime}\right) u_{h} \otimes u_{h^{\prime}} \\
& { }^{\prime \prime} r(y)=\sum_{h, h^{\prime} \in H} d\left(0 ; h, h^{\prime}\right) v_{h} \otimes v_{h^{\prime}}
\end{aligned}
$$

Then the lemma will follow once we show that $c\left(0 ; h, h^{\prime}\right)=d\left(0 ; h, h^{\prime}\right)$, for all $h, h^{\prime} \in$ $H$. Write

$$
c^{\prime}\left(n ; h, h^{\prime}\right)=\pi^{\left(p\left(u_{h^{\prime}}\right)+n p(i)\right) p\left(u_{h}\right)} q^{\left(\left|u_{h^{\prime}}\right|+n \alpha_{i},\left|u_{h}\right|\right)} c\left(n ; h, h^{\prime}\right)
$$

and

$$
d^{\prime}\left(n ; h, h^{\prime}\right)=\pi^{\left(p\left(v_{h^{\prime}}\right)+n p(i)\right) p\left(v_{h}\right)} q^{\left(\left|v_{h^{\prime}}\right|+n \alpha_{i},\left|v_{h}\right|\right)} d\left(n ; h, h^{\prime}\right) .
$$

Note that $p\left(v_{h}\right)=p\left(u_{h}\right)$ and $\left|v_{h}\right|=s_{i}\left(\left|u_{h}\right|\right)$. Since $(-,-)$ on $Q$ is $W$-invariant, $c\left(0 ; h, h^{\prime}\right)=d\left(0 ; h, h^{\prime}\right)$ if and only if $c^{\prime}\left(0 ; h, h^{\prime}\right)=d^{\prime}\left(0 ; h, h^{\prime}\right)$ for all $h, h^{\prime} \in H$.

Using (2.28), we have

$$
\begin{aligned}
& \Delta(x)=\sum_{n \geq 0 ; h, h^{\prime} \in H} c^{\prime}\left(n ; h, h^{\prime}\right) u_{h} \tilde{J}_{\left|u_{h^{\prime}}\right|+n \alpha_{i}^{\vee}} \tilde{K}_{\left|u_{h^{\prime}}\right|+n \alpha_{i}^{\vee}} \otimes u_{h^{\prime}} E_{i}^{(n)} \\
& \Delta(y)=\sum_{n \geq 0 ; h, h^{\prime} \in H} d^{\prime}\left(n ; h, h^{\prime}\right) E_{i}^{(n)} v_{h} \tilde{J}_{\left|v_{h^{\prime}}\right|} \tilde{K}_{\left|v_{h^{\prime}}\right|} \otimes v_{h^{\prime}} .
\end{aligned}
$$

Then, $\Delta(x)=\Delta\left(T_{i}(y)\right)$ by definition. Therefore, applying $T_{i}^{-1} \otimes T_{i}^{-1}$ to (a) gives

$$
\begin{aligned}
& \left(T_{i}^{-1} \otimes T_{i}^{-1}\right) \Delta\left(T_{i}(y)\right) \\
& =\sum_{n \geq 0 ; h, h^{\prime} \in H} c^{\prime}\left(n ; h, h^{\prime}\right)(-1)^{n} q_{i}^{n(n-1)} v_{h} \tilde{J}_{\left|v_{h^{\prime}}\right|-n \alpha_{i}^{\vee}} \tilde{K}_{\left|v_{h^{\prime}}\right|-n \alpha_{i}^{\vee}} \otimes v_{h^{\prime}} F_{i}^{(n)} \tilde{K}_{-n \alpha_{i}^{\vee}},
\end{aligned}
$$

where we have used the fact that $s_{i}\left(\left|u_{h^{\prime}}\right|\right)=\left|v_{h^{\prime}}\right|$.

Let $M=M(\lambda)$ be a Verma module, and let ${ }^{\omega} M$ be the corresponding contragradient module with generator $\xi \in{ }^{\omega} M$ satisfying $F_{i} \xi=0$. Now, by Lemma 3.4.3. $\left(T_{i}^{-1} \otimes T_{i}^{-1}\right) \Delta\left(T_{i}(y)\right)=L_{i} \Delta(y) L_{i}^{-1}$ as maps on ${ }^{\omega} M \otimes{ }^{\omega} M$. Equivalently, as maps we have

$$
\begin{gathered}
\left(\sum_{n \geq 0 ; h, h^{\prime} \in H} c^{\prime}\left(n ; h, h^{\prime}\right)(-1)^{n} q_{i}^{n(n-1)} v_{h} \tilde{J}_{\left|v_{h^{\prime}}\right|-n \alpha_{i}^{\vee}} \tilde{K}_{\left|v_{h^{\prime}}\right|-n \alpha_{i}^{\vee}} \otimes v_{h^{\prime}} F_{i}^{(n)} \tilde{K}_{-n \alpha_{i}^{\vee}}\right) L_{i} \\
=L_{i}\left(\sum_{n \geq 0 ; h, h^{\prime} \in H} d^{\prime}\left(n ; h, h^{\prime}\right) E_{i}^{(n)} v_{h} \tilde{J}_{\left|v_{h^{\prime}}\right|} \tilde{K}_{\left|v_{h^{\prime}}\right|} \otimes v_{h^{\prime}}\right) .
\end{gathered}
$$


Now we apply the equality (c) above to the vector $\xi \otimes \xi \in{ }^{\omega} M \otimes{ }^{\omega} M$. Since $\xi \otimes \xi$ is fixed by $L_{i}$ and $F_{i} \xi=0$, the left-hand side becomes

$$
\begin{aligned}
& \text { LHS }=\sum_{n \geq 0 ; h, h^{\prime} \in H} c^{\prime}\left(n ; h, h^{\prime}\right)(-1)^{n} q_{i}^{n(n-1)} v_{h} \\
& \quad \times\left(\tilde{J}_{\left|v_{h^{\prime}}\right|-n \alpha_{i}^{\vee}} \tilde{K}_{\left|v_{h^{\prime}}\right|-n \alpha_{i}^{\vee}} \otimes v_{h^{\prime}} F_{i}^{(n)} \tilde{K}_{-n \alpha_{i}^{\vee}}\right) L_{i}(\xi \otimes \xi) \\
& =\sum_{n \geq 0 ; h, h^{\prime} \in H} c^{\prime}\left(n ; h, h^{\prime}\right)(-1)^{n} q_{i}^{n(n-1)} v_{h} \tilde{J}_{\left|v_{h^{\prime}}\right|-n \alpha_{i}^{\vee}} \tilde{K}_{\left|v_{h^{\prime}}\right|-n \alpha_{i}^{\vee}} \xi \otimes v_{h^{\prime}} F_{i}^{(n)} \tilde{K}_{-n \alpha_{i}^{\vee}} \xi \\
& =\sum_{h, h^{\prime} \in H} c^{\prime}\left(0 ; h, h^{\prime}\right) v_{h} \tilde{J}_{\left|v_{h^{\prime}}\right|} \tilde{K}_{\left|v_{h^{\prime}}\right|} \xi \otimes v_{h^{\prime}} \xi
\end{aligned}
$$

We also have that the right-hand side becomes

$$
\begin{gathered}
R H S=L_{i}\left(\sum_{n \geq 0 ; h, h^{\prime} \in H} d^{\prime}\left(n ; h, h^{\prime}\right) E_{i}^{(n)} v_{h} \tilde{J}_{\left|v_{h^{\prime}}\right|} \tilde{K}_{\left|v_{h^{\prime}}\right|} \otimes v_{h^{\prime}}\right)(\xi \otimes \xi) \\
=\sum_{n, t \geq 0 ; h, h^{\prime} \in H} d^{\prime}\left(n ; h, h^{\prime}\right)(-1)^{t} \pi_{i}^{t}\left(\pi_{i} q_{i}\right)^{\left(\begin{array}{l}
t \\
2
\end{array}\right)\left(\pi q-q^{-1}\right)^{t}[t]_{i}^{!}} \\
\quad \times F_{i}^{(t)} E_{i}^{(n)} v_{h} \tilde{J}_{\left|v_{h^{\prime}}\right|} \tilde{K}_{\left|v_{h^{\prime}}\right|} \xi \otimes E_{i}^{(t)} v_{h^{\prime}} \xi
\end{gathered}
$$

Let

$$
\varpi:{ }^{\omega} M \longrightarrow{ }^{\omega} M / E_{i}{ }^{\omega} M
$$

be the canonical projection. Applying $1 \otimes \varpi$ to $(\mathrm{d})$, we see that the right-hand side is nonzero in ${ }^{\omega} M \otimes\left({ }^{\omega} M / E_{i}{ }^{\omega} M\right)$ only if $t=0$. Therefore, in ${ }^{\omega} M \otimes\left({ }^{\omega} M / E_{i}{ }^{\omega} M\right)$,

$$
\sum_{h, h^{\prime} \in H} c^{\prime}\left(n ; h, h^{\prime}\right) E_{i}^{(n)} v_{h} \xi \otimes \varpi\left(v_{h^{\prime}}\right)=\sum_{h, h^{\prime} \in H} d^{\prime}\left(0 ; h, h^{\prime}\right) v_{h} \xi \otimes \varpi\left(v_{h^{\prime}} \xi\right) .
$$

Since ${ }^{\omega} M$ is a free $\mathbf{U}^{+}$-module, Corollary 4.2 .7 implies that $E_{i}^{(n)} v_{h} \xi \in{ }^{\omega} M$ are linearly independent for all $n \geq 0$ and $h \in H$. In particular, we must have

$$
\sum_{h^{\prime} \in H}\left(d^{\prime}\left(0 ; h, h^{\prime}\right)-c^{\prime}\left(0 ; h, h^{\prime}\right)\right) v_{h^{\prime}} \xi \in E_{i}{ }^{\omega} M
$$

for each $h \in H$. But, $\mathbf{U}_{J}^{+}[i] \cap E_{i} \mathbf{U}_{J}^{+}=0$, so we conclude $d^{\prime}\left(0 ; h, h^{\prime}\right)=c^{\prime}\left(0 ; h, h^{\prime}\right)$ for all $h, h^{\prime} \in H$. This proves the result.

4.3. Computations with the Inner Product. Recall the inner product on $\mathbf{U}_{J}^{+}$ that was defined in 4.1

Lemma 4.3.1. Assume that $m+m^{\prime}=-a_{i j}$. Then,

$$
\pi_{i}^{\left(\begin{array}{c}
m \\
2
\end{array}\right)}(e(i, j ; m), e(i, j ; m))=\pi_{i}^{\left(\begin{array}{c}
m^{\prime} \\
2
\end{array}\right)}\left(e^{\prime}(i, j ; m), e^{\prime}(i, j ; m)\right)
$$


Proof. By Proposition 4.2.6(a) and (2.16), we have that $\left(e(i, j ; m), E_{i} \mathbf{U}_{J}^{+}\right)=0$. Thus we have

$$
\begin{aligned}
& (e(i, j ; m), e(i, j ; m))=\left(e(i, j ; m), E_{j} E_{i}^{(m)}\right) \\
& \quad=\left(r(e(i, j ; m)), E_{j} \otimes E_{i}^{(m)}\right) \\
& \left.\quad=\prod_{h=0}^{m-t-1}\left(1-\pi_{i}^{h+1-m} q_{i}^{2 h+2-2\left(m-a_{i j}\right.}\right)\right)\left(E_{i}^{(m)}, E_{i}^{(m)}\right)\left(E_{j}, E_{j}\right) \\
& \quad=\pi_{i}^{\left(\begin{array}{c}
m \\
2
\end{array}\right)} \prod_{h=1}^{m} \frac{1-\pi_{i}^{h-m} q_{i}^{2 h+2 m^{\prime}}}{1-\pi_{i}^{h} q_{i}^{2 h}}\left(E_{j}, E_{j}\right) \\
& \quad=\pi_{i}^{\left(\begin{array}{c}
m \\
2
\end{array}\right)} q_{i}^{m m^{\prime}} \prod_{h=1}^{m} \frac{\left(\pi_{i} q_{i}\right)^{h+m^{\prime}}-q_{i}^{-h-m^{\prime}}}{\pi_{i}^{h} q_{i}^{h}-q_{i}^{-h}}\left(E_{j}, E_{j}\right) \\
& \quad=\pi_{i}^{\left(\begin{array}{c}
m \\
2
\end{array}\right)} q_{i}^{m m^{\prime}}\left[\begin{array}{c}
m+m^{\prime} \\
m
\end{array}\right]_{i}\left(E_{j}, E_{j}\right)
\end{aligned}
$$

Similarly, we compute

$$
\left(e^{\prime}\left(i, j ; m^{\prime}\right), e^{\prime}\left(i, j ; m^{\prime}\right)\right)=\pi_{i}^{\left(\begin{array}{c}
m^{\prime} \\
2
\end{array}\right)} q_{i}^{m^{\prime} m}\left[\begin{array}{c}
m+m^{\prime} \\
m^{\prime}
\end{array}\right]_{i}\left(E_{j}, E_{j}\right) .
$$

The lemma follows.

Proposition 4.3.2. For any $x, y \in \mathbf{U}_{J}^{+}[i]$ we have

$$
\left.\pi_{2}^{\left(\left.\right|_{i} ^{-1}(x) \mid\right.}\right)\left(T_{i}^{-1}(x), T_{i}^{-1}(y)\right)=\pi_{2}^{\left(\begin{array}{cc}
|x| \\
2
\end{array}\right)}(x, y),
$$

where, for each $\nu=\sum \nu_{i} \alpha_{i} \in Q,\left(\begin{array}{c}\nu \\ 2\end{array}\right)=\sum\left(\begin{array}{c}\nu_{i} \\ 2\end{array}\right) d_{i}$.

Proof. Indeed, assume $x^{\prime}, x^{\prime \prime} \in \mathbf{U}_{J}^{+}$are such that

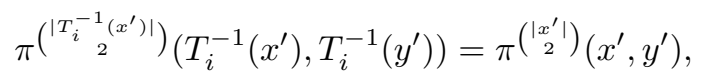

and

$$
\left.\pi_{2}^{\left(\left|T_{i}^{-1}\left(x^{\prime \prime}\right)\right|\right.}\right)\left(T_{i}^{-1}\left(x^{\prime \prime}\right), T_{i}^{-1}\left(y^{\prime \prime}\right)\right)=\pi^{\left(\begin{array}{c}
\left|x^{\prime \prime}\right| \\
2
\end{array}\right)}\left(x^{\prime \prime}, y^{\prime \prime}\right),
$$

for any $y^{\prime}, y^{\prime \prime} \in \mathbf{U}_{J}^{+}[i]$. We show that

$$
\left.\pi^{\left(T_{i}^{-1}\left(x^{\prime} x^{\prime \prime}\right) \mid\right.}\right)\left(T_{i}^{-1}\left(x^{\prime \prime}\right), T_{i}^{-1}(y)\right)=\pi^{\left(\begin{array}{c}
\left|x^{\prime} x^{\prime \prime}\right| \\
2
\end{array}\right)}\left(x^{\prime \prime}, y\right),
$$

for any $y \in \mathbf{U}_{J}^{+}[i]$.

By definition, we have

$$
\left(x^{\prime} x^{\prime \prime}, y\right)=\left(x^{\prime} \otimes x^{\prime \prime}, r(y)\right)
$$

and

$$
\left(T_{i}^{-1}\left(x^{\prime} x^{\prime \prime}\right), T_{i}^{-1}(y)\right)=\left(T_{i}^{-1}\left(x^{\prime}\right) \otimes T_{i}^{-1}\left(x^{\prime \prime}\right), r\left(T_{i}^{-1}(y)\right)\right) .
$$

We have $\left(x^{\prime}, \mathbf{U}_{J}^{+} E_{i}\right)=0$ since $r_{i}\left(x^{\prime}\right)=0$, and so

$$
\left(x^{\prime} \otimes x^{\prime \prime}, r(y)\right)=\left(x^{\prime} \otimes x^{\prime \prime},{ }^{\prime} r(y)\right) .
$$

Also, $\left(T_{i}^{-1}\left(x^{\prime \prime}\right), E_{i} \mathbf{U}_{J}^{+}\right)=0$ since ${ }_{i} r\left(T_{i}^{-1}\left(x^{\prime \prime}\right)\right)=0$, hence

$$
\begin{aligned}
\left(T_{i}^{-1}\left(x^{\prime}\right) \otimes T_{i}^{-1}\left(x^{\prime \prime}\right), r\left(T_{i}^{-1}(y)\right)\right. & =\left(T_{i}^{-1}\left(x^{\prime}\right) \otimes T_{i}^{-1}\left(x^{\prime \prime}\right),{ }^{\prime \prime} r\left(T_{i}^{-1}(y)\right)\right. \\
& =\left(T_{i}^{-1}\left(x^{\prime}\right) \otimes T_{i}^{-1}\left(x^{\prime \prime}\right),\left(T_{i}^{-1} \otimes T_{i}^{-1}\right)^{\prime} r((y)),\right.
\end{aligned}
$$


which follows from Lemma 4.2.10, Write ${ }^{\prime} r(y)=\sum y_{(1)} \otimes y_{(2}$. Then,

$$
\left(x^{\prime} \otimes x^{\prime \prime}, r(y)\right)=\sum\left(x^{\prime}, y_{(1)}\right)\left(x^{\prime \prime}, y_{(2)}\right) \text {. }
$$

Since $T_{i}^{-1}$ is an even algebra homomorphism on $\mathbf{U}$, we have

$$
T_{i}^{-1} \otimes T_{i}^{-1}\left(\sum y_{(1)} \otimes y_{(2)}\right)=\sum T_{i}^{-1}\left(y_{(1)}\right) \otimes T_{i}^{-1}\left(y_{(2)}\right) .
$$

In particular, we see that

$$
\left(T_{i}^{-1}\left(x^{\prime}\right) \otimes T_{i}^{-1}\left(x^{\prime \prime}\right), r\left(T_{i}^{-1}(y)\right)=\sum\left(T_{i}^{-1}\left(x^{\prime}\right), T_{i}^{-1}\left(y_{(1)}\right)\right)\left(T_{i}^{-1}\left(x^{\prime \prime}\right), T_{i}^{-1}\left(y_{(2)}\right)\right) .\right.
$$

Now, for all $z \in \mathbf{U}^{+}{ }_{J}[i]$, if

$$
|z|=\sum_{j \in I} \nu_{j} \alpha_{j} \quad \text { and } \quad\left|T_{i}^{-1}(z)\right|=\sum_{j \in I} \nu_{j}^{\prime} \alpha_{j}
$$

then $\nu_{j} p(j) \equiv \nu_{j}^{\prime} p(j)(\bmod 2)$ for all $j \in I$. Hence,

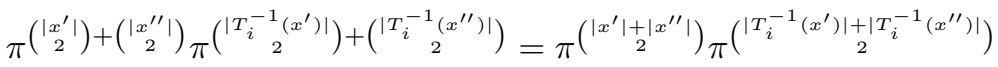

$$
\begin{aligned}
& =\pi^{\left(\begin{array}{c}
\left|x^{\prime} x^{\prime \prime}\right| \\
2
\end{array}\right)} \pi^{\left(\begin{array}{c}
\left|T_{i}^{-1}\left(x^{\prime} x^{\prime \prime}\right)\right| \\
2
\end{array}\right) .}
\end{aligned}
$$

Then by the induction hypothesis,

$$
\begin{aligned}
& \left(T_{i}^{-1}\left(x^{\prime} x^{\prime \prime}\right), T_{i}^{-1}(y)\right)=\sum\left(T_{i}^{-1}\left(x^{\prime}\right), T_{i}^{-1}\left(y_{(1)}\right)\right)\left(T_{i}^{-1}\left(x^{\prime \prime}\right), T_{i}^{-1}\left(y_{(2)}\right)\right)
\end{aligned}
$$

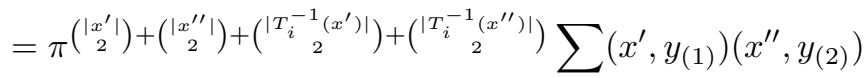

$$
\begin{aligned}
& =\pi{ }^{\left(\begin{array}{c}
\left|x^{\prime} x^{\prime \prime}\right| \\
2
\end{array}\right)} \pi^{\left({ }^{\left(T_{i}^{-1}\left(x^{\prime} x^{\prime \prime}\right) \mid\right.}\right)} \sum\left(x^{\prime}, y_{(1)}\right)\left(x^{\prime \prime}, y_{(2)}\right) \\
& =\pi^{\left(\begin{array}{c}
\left|x^{\prime} x^{\prime \prime}\right| \\
2
\end{array}\right)} \pi^{\left({ }^{\left|T_{i}^{-1}\left(x^{\prime} x^{\prime \prime}\right)\right|}\right)}\left(x^{\prime} x^{\prime \prime}, y\right) .
\end{aligned}
$$

Finally, we have reduced to checking that the proposition holds for $x$ a generator of $\mathbf{U}_{J}^{+}[i]$ (i.e. $\left.x=e(i, j ; m)\right)$. We may assume that $y$ is homogeneous of the same weight as $x$. Since $y \in \mathbf{U}_{J}^{+}[i]$, this forces $y$ to be a scalar multiple of $e(i, j ; m)$. Therefore, the proposition follows from Lemma 4.3.1.

Definition 4.3.3. A sequence $\mathbf{h}=\left(i_{1}, \ldots, i_{n}\right) \in I^{n}$ is said to be admissible if, for any $1 \leq a \leq b \leq n$,

(a) $T_{i_{a}} T_{i_{a+1}} \cdots T_{i_{b-1}}\left(E_{i_{b}}\right) \in \mathbf{U}_{J}^{+}$, and

(b) $T_{i_{b}}^{-1} T_{i_{b-1}}^{-1} \cdots T_{i_{a+1}}^{-1}\left(E_{i_{a}}\right) \in \mathbf{U}_{J}^{+}$.

Now, assume $\mathbf{h}$ is admissible, and $1 \leq p \leq n$. We say that $x \in \mathbf{U}_{J}^{+}$is adapted to $(\mathbf{h}, p)$ if,

(c) $T_{i_{a}} T_{i_{a+1}} \cdots T_{i_{p}}(x) \in \mathbf{U}_{J}^{+}$, for any $1 \leq a \leq p$, and

(d) $T_{i_{b}}^{-1} T_{i_{b-1}}^{-1} \cdots T_{i_{p+1}}^{-1}(x) \in \mathbf{U}_{J}^{+}$, for any $p+1 \leq b \leq n$.

Given $x \in \mathbf{U}_{J}^{+}$adapted to $(\mathbf{h}, p)$ as above, and a sequence $\mathbf{c}=\left(c_{1}, \ldots, c_{n}\right) \in \mathbb{N}_{n}$, define

$$
\begin{aligned}
L(\mathbf{h}, \mathbf{c}, p, x)= & E_{i_{p+1}}^{\left(c_{p+1}\right)} \cdot\left[T_{i_{p+1}}\left(E_{i_{p+2}}^{\left(c_{p+2}\right)}\right)\right] \cdots\left[T_{i_{p+1}} T_{i_{p+2}} \cdots T_{i_{n-1}}\left(E_{i_{n}}^{\left(c_{n}\right)}\right)\right] \\
& \cdot x \cdot\left[T_{i_{p}}^{-1} T_{i_{p-1}}^{-1} \cdots T_{i_{2}}^{-1}\left(E_{i_{1}}^{\left(c_{1}\right)}\right)\right] \cdots\left[T_{i_{p}}^{-1}\left(E_{i_{p-1}}^{\left(c_{p-1}\right)}\right)\right] \cdot E_{i_{p}}^{\left(c_{p}\right)} .
\end{aligned}
$$

Then, by definition, $L(\mathbf{h}, \mathbf{c}, p, x) \in \mathbf{U}_{J}^{+}$. 
Proposition 4.3.4. Let $\mathbf{c}=\left(c_{1}, c_{2}, \ldots, c_{n}\right), \mathbf{c}^{\prime}=\left(c_{1}^{\prime}, c_{2}^{\prime}, \ldots, c_{n}^{\prime}\right) \in \mathbb{N}^{n}$. Let $\mathbf{h} \in I^{n}$ be admissible and suppose $x, x^{\prime} \in \mathbf{U}_{J}^{+}$is adapted to $(\mathbf{h}, p)$ for some $1 \leq p \leq n$. Then there exists $\ell(\mathbf{h}, \mathbf{c}, p, x) \in \mathbb{Z}$ such that

$$
\left(L(\mathbf{h}, \mathbf{c}, p, x), L\left(\mathbf{h}, \mathbf{c}^{\prime}, p, x^{\prime}\right)\right)=\pi^{\ell(\mathbf{h}, \mathbf{c}, p, x)}\left(x, x^{\prime}\right) \prod_{s=1}^{n}\left(E_{i_{s}}^{\left(c_{s}\right)}, E_{i_{s}}^{\left(c_{s}^{\prime}\right)}\right) .
$$

Proof. For any $i \in I, t, t^{\prime} \in \mathbb{N}$ and $y, y^{\prime} \in \mathbf{U}_{J}^{+}[i]$, we have

$$
\left(E_{i}^{(t)} y, E_{i}^{\left(t^{\prime}\right)} y^{\prime}\right)=\left(E_{i}^{(t)}, E_{i}^{\left(t^{\prime}\right)}\right)\left(y, y^{\prime}\right)
$$

Similarly, if $z, z^{\prime} \in{ }^{\sigma} \mathbf{U}_{J}^{+}[i]$, we have

$$
\left(z E_{i}^{(t)}, z^{\prime} E_{i}^{\left(t^{\prime}\right)}\right)=\left(E_{i}^{(t)}, E_{i}^{\left(t^{\prime}\right)}\right)\left(z, z^{\prime}\right) .
$$

Suppose $p<n$ and the proposition holds for $p+1$. Let $\tilde{\mathbf{c}}, \tilde{\mathbf{c}}^{\prime}$ be the sequences defined by $\tilde{c}_{p+1}=\tilde{c}_{p+1}^{\prime}=0, \tilde{c}_{s}=c_{s}$ and ${\tilde{c_{s}}}^{\prime}=c_{s}^{\prime}$ for $s \neq p+1$. Let $\tilde{x}=T_{i_{p+1}}^{-1}(x)$, $\tilde{x}^{\prime}=T_{i_{p+1}}^{-1}\left(x^{\prime}\right)$, and $\tilde{p}=p+1$. Then $\tilde{x}$ is adapted to $(\mathbf{h}, \tilde{p})$ and

$$
L(\mathbf{h}, \mathbf{c}, p, x)=E_{i_{p+1}}^{\left(c_{p+1}\right)} T_{i_{p+1}}(L(\mathbf{h}, \tilde{\mathbf{c}}, \tilde{p}, \tilde{x})) .
$$

By assumption, we have $T_{i_{p+1}}(L(\mathbf{h}, \tilde{\mathbf{c}}, \tilde{p}, \tilde{x})) \in \mathbf{U}_{J}^{+}$. In particular, this implies that $T_{i_{p+1}}(L(\mathbf{h}, \tilde{\mathbf{c}}, \tilde{p}, \tilde{x})) \in \mathbf{U}_{J}^{+}\left[i_{p+1}\right]$ and $L(\mathbf{h}, \tilde{\mathbf{c}}, \tilde{p}, \tilde{x}) \in{ }^{\sigma} \mathbf{U}_{J}^{+}\left[i_{p+1}\right]$. Similarly,

$$
L\left(\mathbf{h}, \mathbf{c}^{\prime}, p, x\right)=E_{i_{p+1}}^{\left(c_{p+1}^{\prime}\right)} T_{i_{p+1}}\left(L\left(\mathbf{h}, \tilde{\mathbf{c}}^{\prime}, \tilde{p}, \tilde{x}\right)\right) \quad \text { and } \quad L\left(\mathbf{h}, \tilde{\mathbf{c}}^{\prime}, \tilde{p}, \tilde{x}\right) \in{ }^{\sigma} \mathbf{U}_{J}^{+}\left[i_{p+1}\right] .
$$

Let $\nu=|L(\mathbf{h}, \tilde{\mathbf{c}}, \tilde{p}, \tilde{x})|$. Then using $(\star)$, Proposition 4.3.2, and the induction hypothesis, we see that

$$
\begin{aligned}
(L(\mathbf{h}, \mathbf{c}, p, x), L & \left.\left(\mathbf{h}, \mathbf{c}^{\prime}, p, x\right)\right) \\
& =\left(E_{i_{p+1}}^{\left(c_{p+1}\right)}, E_{i_{p+1}}^{\left(c_{p+1}^{\prime}\right)}\right)\left(T_{i_{p+1}}(L(\mathbf{h}, \tilde{\mathbf{c}}, \tilde{p}, \tilde{x})), T_{i_{p+1}}\left(L\left(\mathbf{h}, \tilde{\mathbf{c}}^{\prime}, \tilde{p}, \tilde{x}\right)\right)\right) \\
& =\pi^{\left(\begin{array}{c}
\nu \\
2
\end{array}\right)+\left({ }^{s_{i}(\nu)}\right)}\left(E_{i_{p+1}}^{\left(c_{p+1}\right)}, E_{i_{p+1}}^{\left(c_{p+1}^{\prime}\right)}\right)\left(L(\mathbf{h}, \tilde{\mathbf{c}}, \tilde{p}, \tilde{x}), L\left(\mathbf{h}, \tilde{\mathbf{c}}^{\prime}, \tilde{p}, \tilde{x}\right)\right) \\
& =\pi^{\ell(\mathbf{h}, \mathbf{c}, p, x)+\left(\begin{array}{c}
\nu \\
2
\end{array}\right)+\left({ }^{s_{i_{p+1}}(\nu)}\right)}\left(x, x^{\prime}\right) \prod_{s=1}^{n}\left(E_{i_{s}}^{\left(c_{s}\right)}, E_{i_{s}}^{\left(c_{s}^{\prime}\right)}\right)
\end{aligned}
$$

Therefore, it suffices to assume $p=n$, whence

$$
L(\mathbf{h}, \mathbf{c}, n, x)=x \cdot\left[T_{i_{n}}^{-1} T_{i_{n-1}}^{-1} \cdots T_{i_{2}}^{-1}\left(E_{i_{1}}^{\left(c_{1}\right)}\right)\right] \cdots\left[T_{i_{n}}^{-1}\left(E_{i_{n-1}}^{\left(c_{n-1}\right)}\right)\right] \cdot E_{i_{n}}^{\left(c_{n}\right)} .
$$

When $n=0$, the result is trivial. Now assume $n>0$ and suppose the result holds for $n-1$. Let $\tilde{x}=T_{i_{n}}(x), T_{i}\left(x^{\prime}\right)=\tilde{x}^{\prime}, \tilde{\mathbf{h}}=\left(i_{1}, \ldots, i_{n-1}\right)$, and $\tilde{\mathbf{c}}=\left(c_{1}, \ldots, c_{n-1}\right)$. Then

$$
\begin{aligned}
L(\mathbf{h}, \mathbf{c}, n, x) & =T_{i_{n}}^{-1}(L(\tilde{\mathbf{h}}, \tilde{\mathbf{c}}, n-1, \tilde{x})) E_{i_{n}}^{\left(c_{n}\right)}, \\
L\left(\mathbf{h}, \mathbf{c}^{\prime}, n, x^{\prime}\right) & =T_{i_{n}}^{-1}\left(L\left(\tilde{\mathbf{h}}, \tilde{\mathbf{c}}^{\prime}, n-1, \tilde{x}^{\prime}\right)\right) E_{i_{n}}^{\left(c_{n}^{\prime}\right)} .
\end{aligned}
$$

Then as before, we apply $(\star \star)$, Proposition 4.3.2, and the induction hypothesis to obtain

$$
\begin{aligned}
\left(L(\mathbf{h}, \mathbf{c}, n, x), L\left(\mathbf{h}, \mathbf{c}^{\prime}, n, x\right)\right) \\
=\left(E_{i_{n}}^{\left(c_{i_{n}}\right)}, E_{i_{n}}^{\left(c_{i_{n}}^{\prime}\right)}\right)\left(T_{i_{n}}(L(\mathbf{h}, \tilde{\mathbf{c}}, n-1, \tilde{x})), T_{i_{n}}\left(L\left(\mathbf{h}, \tilde{\mathbf{c}}^{\prime}, n-1, \tilde{x}^{\prime}\right)\right)\right) \\
=\pi^{\ell(\mathbf{h}, \mathbf{c}, n-1, x)+\left(\begin{array}{c}
\nu \\
2
\end{array}\right)+\left(\begin{array}{c}
s_{i_{n}}(\nu) \\
{ }_{2}
\end{array}\right)}\left(x, x^{\prime}\right) \prod_{s=1}^{n}\left(E_{i_{s}}^{\left(c_{s}\right)}, E_{i_{s}}^{\left(c_{s}^{\prime}\right)}\right),
\end{aligned}
$$


where $\nu=|L(\tilde{\mathbf{h}}, \tilde{\mathbf{c}}, n-1, \tilde{x})|$. This finishes the proof.

\section{BRAid GROUP RELATIONS}

5.1. The rank 2 PBW basis. In this section, we assume $|I|=2$ and that $\left[a_{i j}\right]_{i, j \in I}$ is of finite type.

Lemma 5.1.1. Let $i, j \in I, i \neq j$. Then, as automorphisms of ${ }_{\mathcal{A}} \mathbf{U}$,

$$
\underbrace{T_{i} T_{j} T_{i} \cdots}_{m_{i j}}=\underbrace{T_{j} T_{i} T_{j} \cdots}_{m_{i j}}
$$

Proof. We assume $m_{i j} \in\{2,3,4,6\}$ as otherwise there is nothing to prove. Moreover, when both $i, j \in I_{\overline{0}}$, this is $[\mathrm{L}$, Section 39.2]. We may, therefore, assume that either $i$ or $j$ is odd. Then we must have $m_{i j} \in\{2,4\}$ and, if both $i, j \in I_{\overline{1}}$, then $m_{i j}=2$.

First, assume that $m_{i j}=2$, so $\left\langle i, j^{\prime}\right\rangle=\left\langle j, i^{\prime}\right\rangle=0$. Then $T_{j}\left(E_{i}\right)=\tilde{J}_{j}^{p(i)} E_{i}$ and $T_{j}\left(F_{i}\right)=\tilde{J}_{j}^{p(i)} F_{i}$. Therefore, we have

$$
\begin{aligned}
& T_{i} T_{j}\left(E_{i}\right)=T_{i}\left(\tilde{J}_{j}^{p(i)} E_{i}\right)=-\pi_{i} \tilde{J}_{j}^{p(i)} \tilde{J}_{i} \tilde{K}_{i}^{-1} F_{i} \\
& T_{j} T_{i}\left(E_{i}\right)=T_{j}\left(-\pi_{i} \tilde{J}_{i} \tilde{K}_{i}^{-1} F_{i}\right)=-\pi_{i} \tilde{J}_{i} \tilde{K}_{i}^{-1} \tilde{J}_{j}^{p(i)} F_{i}
\end{aligned}
$$

so $T_{i} T_{j}\left(E_{i}\right)=T_{j} T_{i}\left(E_{i}\right)$. By symmetry $T_{i} T_{j}\left(E_{j}\right)=T_{j} T_{i}\left(E_{j}\right)$. By a similar argument, we deduce that $T_{i} T_{j}\left(F_{i}\right)=T_{j} T_{i}\left(F_{i}\right)$ and $T_{i} T_{j}\left(F_{j}\right)=T_{j} T_{i}\left(F_{j}\right)$. It is clear that $T_{i} T_{j}\left(K_{\mu}\right)=T_{j} T_{i}\left(K_{\mu}\right)$. Therefore, the lemma holds in this case.

Now, Assume that $m_{i j}=4$. We may assume $i \in I_{\overline{1}}$ and $j \in I_{\overline{0}}$, so that $\left\langle i, j^{\prime}\right\rangle=-2$ and $\left\langle j, i^{\prime}\right\rangle=-1$. In this case, we have $q_{j}=q_{i}^{2}$. Additionally, we will repeatedly use the fact that

$$
\pi_{i}^{p(j)}=\pi_{j}^{p(i)}=1, \quad \tilde{J}_{i}^{p(j)}=\tilde{J}_{j}^{p(i)}=1 .
$$

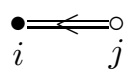

Recall the elements $e_{1, m}=e(i, j ; m)$ and $e_{1, m}^{\prime}=e^{\prime}(i, j ; m)$. Since $\sigma\left(e_{1, m}\right)=e_{1, m}^{\prime}$, Lemma 2.6.1 implies that

$$
-q_{i}^{2-2 m} \pi_{i}^{m+1} e_{1, m}^{\prime} E_{i}+E_{i} e_{1, m}^{\prime}=[m+1]_{i} e_{1, m+1} .
$$

Using Theorem 3.3.1, we have that $T_{i}\left(E_{j}\right)=\pi_{i} e_{1,2}$ and $T_{i}^{-1}\left(E_{j}\right)=\pi_{i} e_{1,2}^{\prime}$. Interchanging the roles of $i$ and $j$ in Theorem 3.3 .1 and using the relations $q_{j}=q_{i}^{2}$ and (a), we have

$$
\begin{aligned}
T_{j}\left(E_{i}\right) & =e_{j, i, 1,1}=E_{i} E_{j}-q_{j} E_{j} E_{i} \\
& =E_{i} E_{j}-q_{i}^{2} E_{j} E_{i}=e_{i, j ; 1,1}^{\prime}=e_{1,1}^{\prime},
\end{aligned}
$$

and similarly $T_{j}^{-1}\left(E_{i}\right)=e_{j, i, 1,1}^{\prime}=e_{i, j ; 1,1}=e_{1,1}$. Therefore, using (b) we have

$$
\begin{aligned}
T_{j}^{-1}\left(e_{1,2}^{\prime}\right) & =[2]_{i}^{-1} T_{j}^{-1}\left(-e_{1,1}^{\prime} E_{i}+E_{i} e_{1,1}^{\prime}\right) \\
& =[2]_{i}^{-1} T_{j}^{-1}\left(-e_{1,1}^{\prime}\right) T_{j}^{-1}\left(E_{i}\right)+T_{j}^{-1}\left(E_{i}\right) T_{j}^{-1}\left(e_{1,1}^{\prime}\right) \\
& =[2]_{i}^{-1}\left(-E_{i} e_{1,1}+e_{1,1} E_{i}\right)=e_{1,2} .
\end{aligned}
$$


It follows that $T_{j}\left(e_{1,2}\right)=e_{1,2}^{\prime}$. By Lemma 4.2.2 and the fact that $p(j)=0$, $T_{i}\left(e_{1,1}^{\prime}\right)=\pi_{i} e_{1,1}$ and, therefore,

$$
E_{j} \stackrel{T_{i}}{\longmapsto} \pi_{i} e_{1,2} \stackrel{T_{j}}{\longrightarrow} \pi_{i} e_{1,2}^{\prime} \stackrel{T_{i}}{\longrightarrow} E_{j}
$$

and

$$
E_{i} \stackrel{T_{j}}{\longrightarrow} e_{1,1}^{\prime} \stackrel{T_{i}}{\longmapsto} \pi_{i} e_{1,2} \stackrel{T_{j}}{\longrightarrow} \tilde{J}_{i} E_{i}
$$

By a similar computation,

$$
T_{i} T_{j} T_{i}\left(F_{j}\right)=F_{j} \quad \text { and } \quad T_{j} T_{i} T_{j}\left(F_{i}\right)=\pi_{i} F_{i} .
$$

Hence,

$$
\begin{aligned}
& T_{j} T_{i} T_{j} T_{i}\left(E_{j}\right)=T_{j}\left(E_{j}\right)=-\tilde{J}_{j} \tilde{K}_{j}^{-1} F_{j}, \\
& T_{i} T_{j} T_{i} T_{j}\left(E_{j}\right)=T_{i} T_{j} T_{i}\left(-\tilde{J}_{j} \tilde{K}_{j}^{-1} F_{j}\right)=-\tilde{J}_{j} \tilde{K}_{j}^{-1} F_{j}, \\
& T_{j} T_{i} T_{j} T_{i}\left(E_{i}\right)=T_{j} T_{i} T_{j}\left(-\pi_{i} \tilde{J}_{i} \tilde{K}_{i}^{-1} F_{i}\right)=-\tilde{J}_{i} \tilde{K}_{i}^{-1} F_{i}, \\
& T_{i} T_{j} T_{i} T_{j}\left(E_{i}\right)=T_{i}\left(\tilde{J}_{i} E_{i}\right)=-\tilde{J}_{i} \tilde{K}_{i}^{-1} F_{i},
\end{aligned}
$$

where we have used that $s_{i} s_{j} s_{i}(j)=j$ in the second line, and $s_{j} s_{i} s_{j}(i)=i$ in the third. Therefore, $T_{i} T_{j} T_{i} T_{j}$ and $T_{j} T_{i} T_{j} T_{i}$ agree on $E_{i}$ and $E_{j}$. By a similar argument, they agree on $F_{i}$ and $F_{j}$. It is easy to prove that they agree on $K_{\mu}$ and $J_{\mu}$, therefore, they are equal. This proves the theorem.

Now let $m=m_{i j}$. Then for any $p$ such that $0 \leq p \leq m-1$,

$$
(\underbrace{\ldots T_{j} T_{i}}_{p \text { factors }})\left(E_{j}\right),(\underbrace{\ldots T_{i} T_{j}}_{p \text { factors }})\left(E_{i}\right),(\underbrace{\ldots T_{j}^{-1} T_{i}^{-1}}_{p \text { factors }})\left(E_{j}\right), \quad \underbrace{\left(\ldots T_{i}^{-1} T_{j}^{-1}\right.}_{p \text { factors }})\left(E_{i}\right) \in \mathbf{U}_{J}^{+} .
$$

In particular, the sequences $\mathbf{i}=(i, j, i, j, \ldots)$ and $\mathbf{j}=(j, i, j, i, \ldots)$ with $m$ terms are admissible sequences. Consider the following sets of elements of $\mathbf{U}_{J}^{+}$, where each element is a product of $m$ elements of $\mathbf{U}_{J}^{+}$and $\mathbf{c}=\left(c_{1}, \ldots, c_{m}\right) \in \mathbb{N}^{m}$ :

$$
\begin{gathered}
\left\{E_{i}^{\left(c_{1}\right)} T_{i}\left(E_{j}^{\left(c_{2}\right)}\right) T_{i} T_{j}\left(E_{i}^{\left(c_{3}\right)}\right) \ldots \mid\left(c_{1}, \ldots, c_{m}\right) \in \mathbb{N}^{m}\right\} ; \\
\left\{E_{j}^{\left(c_{1}\right)} T_{j}\left(E_{i}^{\left(c_{2}\right)}\right) T_{j} T_{i}\left(E_{j}^{\left(c_{3}\right)}\right) \ldots \mid\left(c_{1}, \ldots, c_{m}\right) \in \mathbb{N}^{m}\right\} ; \\
\left\{E_{i}^{\left(c_{1}\right)} T_{i}^{-1}\left(E_{j}^{\left(c_{2}\right)}\right) T_{i}^{-1} T_{j}^{-1}\left(E_{i}^{\left(c_{3}\right)}\right) \ldots \mid\left(c_{1}, \ldots, c_{m}\right) \in \mathbb{N}^{m}\right\} ; \\
\left\{E_{j}^{\left(c_{1}\right)} T_{j}^{-1}\left(E_{i}^{\left(c_{2}\right)}\right) T_{j}^{-1} T_{i}^{-1}\left(E_{j}^{\left(c_{3}\right)}\right) \ldots \mid\left(c_{1}, \ldots, c_{m}\right) \in \mathbb{N}^{m}\right\} .
\end{gathered}
$$

Note that each set consists of elements of the form $\sigma^{e}(L(\mathbf{h}, \mathbf{c}, p, 1))$ where $\mathbf{h}=\mathbf{i}$ or $\mathbf{j}, p=0$ or $m$, and $e=0$ or 1 . In particular, by Proposition 4.3.4 each set consists of pairwise orthogonal elements of $\mathbf{U}_{J}^{+}$; in addition, if $x$ is an element of one of these sets, then $(x, x)$ is not a zero divisor in $\mathbf{U}_{J}^{0}$, and therefore each set is linearly independent.

Lemma 5.1.2. Each of the sets (a)-(d) is a basis of the free $\mathbf{U}_{J}^{0}$-module $\mathbf{U}_{J}^{+}$.

Proof. Because the characters of $\mathbf{U}^{+}$and $\left.\mathbf{U}^{+}\right|_{\pi=1}$ are the same, the proof of this fact is identical to the proof of [L, Lemma 39.3.2]. 
5.2. Proof of the braid relations on modules. Recall that we denote the highest weight vector of $V(\lambda)$ by $\eta_{\lambda}$.

Lemma 5.2.1. Let $\mathbf{h}=\left(i_{1}, \ldots, i_{N}\right)$ be a sequence in $I$ such that $s_{i_{1}} \ldots s_{i_{N}}$ is a reduced expression in $W$. Let $\lambda \in P_{+}$and $a_{k}=\left\langle s_{i_{N}} \ldots s_{i_{k+1}}\left(\alpha_{i_{k}}^{\vee}\right), \lambda\right\rangle$. Then

$$
T_{i_{1}} \ldots T_{i_{N}} \eta_{\lambda}=F_{i_{1}}^{\left(a_{1}\right)} \ldots F_{i_{N}}^{\left(a_{N}\right)} \eta_{\lambda} .
$$

Proof. Note that this is trivially true when $N=0$, and that $N=1$ follows from Lemma 3.1.3. Now assume $N \geq 2$ and let $\eta(\mathbf{h})=T_{i_{1}} \ldots T_{i_{N}} \eta_{\lambda}$. Then by induction, it suffices to show that $T_{i_{1}} \eta\left(\mathbf{h}^{\prime}\right)=F_{i_{1}}^{\left(a_{1}\right)} \eta\left(\mathbf{h}^{\prime}\right)$ where $\mathbf{h}^{\prime}=\left(i_{1}, \ldots, i_{N-1}\right)$.

Let $\mu=s_{i_{2}} \ldots s_{i_{N}}(\lambda)$. Note that $\eta\left(\mathbf{h}^{\prime}\right) \in V(\lambda)_{\mu}$ and $\left\langle\alpha_{i_{1}}^{\vee}, \mu\right\rangle=a_{1}$ by the $W$ invariance of $\langle-,-\rangle$. In particular, if $E_{i_{1}} \eta(\mathbf{h})=0$ then $T_{i_{1}} \eta\left(\mathbf{h}^{\prime}\right)=F_{i_{1}}^{\left(a_{1}\right)} \eta\left(\mathbf{h}^{\prime}\right)$ by Lemma 3.1.3. Therefore, it remains to show that $E_{i_{1}} \eta(\mathbf{h})=0$.

Now note that $E_{i_{1}} \eta\left(\mathbf{h}^{\prime}\right) \in V(\lambda)_{\mu+\alpha_{i_{1}}}$, so it suffices to show this weight space is zero. Assume to the contrary that $V(\lambda)_{\mu+\alpha_{i_{1}}} \neq 0$. Then since $s_{i_{2}} \ldots s_{i_{N}}\left(\mu+\alpha_{i_{1}}\right)=$ $\lambda+s_{i_{N}} \ldots s_{i_{2}}\left(\alpha_{i_{1}}\right)$, we have that $V(\lambda)_{\lambda+s_{i_{N}} \ldots s_{i_{2}}\left(\alpha_{i_{1}}\right)} \neq 0$. But then $s_{i_{N}} \ldots s_{i_{2}}\left(\alpha_{i_{1}}\right)<$ 0 , which contradicts that $s_{i_{1}} \ldots s_{i_{N}}$ is a reduced expression. This completes the proof.

Proposition 5.2.2 (Quantum Verma Identity). Assume that $|I|=2,\left[a_{i j}\right]_{i, j \in I}$ is of finite type, and $p(i) p(j)=0$. Let $\lambda \in P_{+}$. Define

$$
a_{k}=\langle\underbrace{\ldots s_{j} s_{i} s_{j}}_{m-k \text { factors }}\left(\alpha_{i}^{\vee}\right), \lambda\rangle, \quad b_{k}=\langle\underbrace{\ldots s_{i} s_{j} s_{i}}_{m-k \text { factors }}\left(\alpha_{j}^{\vee}\right), \lambda\rangle .
$$

Set $x=F_{i}^{\left(a_{1}\right)} F_{j}^{\left(a_{2}\right)} F_{i}^{\left(a_{3}\right)} \ldots$ and $y=F_{j}^{\left(b_{1}\right)} F_{i}^{\left(b_{2}\right)} F_{j}^{\left(b_{3}\right)} \ldots$ where both products have $m$ factors. Then $x=y$.

Proof. If $i, j \in I_{\overline{0}}$, then the statement of the proposition follows from $\mathrm{L}$, Proposition 39.3.7]. If $\left\langle i, j^{\prime}\right\rangle=0$, then since $p(i) p(j)=0$, the statement is trivially true by the Serre relation $F_{i} F_{j}=F_{j} F_{i}$. Therefore, we may assume $i \in I_{\overline{1}}$ and $m=4$. In this case, a similar proof to Lusztig's can be given, however, we will sketch a shorter proof here by utilizing the theory of twistors from CFLW].

By direct computation we see that $x, y \in \mathbf{U}_{\nu}^{-}$where $\nu=2\left\langle\alpha_{i}^{\vee}+\alpha_{j}^{\vee}, \lambda\right\rangle \alpha_{i}+$ $\left\langle\alpha_{i}^{\vee}+2 \alpha_{j}^{\vee}, \lambda\right\rangle \alpha_{j}$. Moreover, $a_{t}=b_{5-t}$ and so $x=\varrho(y)$, where $\varrho=\omega^{-1} \sigma \omega$. Now set $x=z^{-}$, where $z \in \mathbf{f}$. Then we want to show $z=\varrho(z)$, where we define $\varrho: \mathbf{f} \longrightarrow \mathbf{f}$ by $\varrho\left(z_{1}\right)^{-}=\varrho\left(z_{1}^{-}\right)$for any $z_{1} \in \mathbf{f}$.

Let $\left.\mathbf{f}\right|_{\pi= \pm 1}$ denote the quotient of $\mathbf{f}$ by the two-sided ideal generated by $\pi \mp 1$; in particular, note that $\mathbf{f}=(1+\pi) \mathbf{f} \oplus(1-\pi) \mathbf{f}=\left.\left.\mathbf{f}\right|_{\pi=1} \oplus \mathbf{f}\right|_{\pi=-1}$ as algebras. Let $\left.(-)\right|_{\pi= \pm 1}$ be the canonical projections and note that $\left.\mathbf{f}\right|_{\pi=1}$ is identically Lusztig's half quantum group. In particular, $\mathrm{L}$, Proposition 39.3.7] implies $\varrho\left(\left.z\right|_{\pi=1}\right)=\left.z\right|_{\pi=1}$, so it suffices to prove that $\varrho\left(\left.z\right|_{\pi=-1}\right)=\left.z\right|_{\pi=-1}$.

Let $\mathbf{t}^{2}=-1$. By CFLW] Theorem 2.4], there exists a $\mathbb{Q}(\mathbf{t})$-linear bijection $\mathfrak{X}$ between (scalar extensions of) $\left.\mathbf{f}\right|_{\pi=1}$ and $\left.\mathbf{f}\right|_{\pi=-1}$. In particular,

$$
\mathfrak{X}\left(\left.z\right|_{\pi=1}\right)=\left.\mathbf{t}^{n} z\right|_{\pi=-1}
$$

for some $n \in \mathbb{Z}$. Utilizing Proposition 2.6 of loc. cit., we have $\mathfrak{X}\left(\varrho\left(\left.z\right|_{\pi=1}\right)\right)=$ $(-1)^{n^{\prime}} \mathbf{t}^{n} \varrho\left(\left.z\right|_{\pi=-1}\right)$ for some $n^{\prime} \in \mathbb{Z}$. However, there is an explicit formula for $n^{\prime}$ 
depending on $|z|=\nu$, and it can be computed directly that $n^{\prime} \in 2 \mathbb{Z}$. On the other hand,

$$
\left.\mathbf{t}^{n} z\right|_{\pi=-1}=\mathfrak{X}\left(\left.z\right|_{\pi=1}\right)=\mathfrak{X}\left(\varrho\left(\left.z\right|_{\pi=1}\right)\right)=\mathbf{t}^{n} \varrho\left(\left.z\right|_{\pi=-1}\right),
$$

and hence $\left.z\right|_{\pi=-1}=\varrho\left(\left.z\right|_{\pi=-1}\right)$ as desired.

Consider the case $I=I_{\overline{1}}=\{i, j\}$ and $\left\langle\alpha_{i}^{\vee}, \alpha_{j}\right\rangle=0$. We note that Proposition 5.2 .2 is not true in this case. Indeed, if $\left\langle\alpha_{i}^{\vee}, \alpha_{j}\right\rangle=0$ then we have the Serre relation $F_{i} F_{j}=\pi F_{j} F_{i}$, and so in general we have the identity

$$
F_{i}^{(a)} F_{j}^{(b)}=\pi^{a b} F_{j}^{(b)} F_{i}^{(a)} .
$$

Lemma 5.2.3. Assume that $|I|=2$ and that $\left[a_{i j}\right]_{i, j \in I}$ is of finite type. Let $M$ be an integrable $\mathbf{U}$-module.

(1) Assume $p(i) p(j)=0$. Then we have

$$
T_{i} T_{j} T_{i} \ldots=T_{j} T_{i} T_{j} \ldots: M \rightarrow M,
$$

where both products have $m$ factors.

(2) Assume $p(i)=p(j)=1$, so that $\left\langle\alpha_{i}^{\vee}, \alpha_{j}\right\rangle=\left\langle\alpha_{j}^{\vee}, \alpha_{i}\right\rangle=0, m=2$, and $P$ can be identified with $\mathbb{Z} \times \mathbb{Z}$. Then for $s, t \in \mathbb{Z}$,

$$
T_{i} T_{j}=\pi^{s t} T_{j} T_{i}: M_{s, t} \rightarrow M_{-s,-t} .
$$

Proof. The statement (1) is proved identically to [L, Lemma 39.4.1], whereas (2) follows from a slightly modified proof. Indeed, assume $p(i)=p(j)=1$ and let us identify weights with $\mathbb{Z} \times \mathbb{Z}$ (where the first component corresponds to $\alpha_{i}$, and the second corresponds to $\alpha_{j}$ ). Let $x \in M_{s, t}$, and without loss of generality we may assume that $x=u \eta$, where $E_{i} \eta=E_{j} \eta=0$. Suppose first that $u=1$. Then by Lemma 5.2.1 we have $T_{i} T_{j}(\eta)=F_{i}^{(s)} F_{j}^{(t)} \eta$ and $T_{j} T_{i}(\eta)=F_{j}^{(t)} F_{i}^{(s)} \eta$. Then (5.1) implies $T_{i} T_{j}(\eta)=\pi^{s t} T_{j} T_{i}(\eta)$.

Now suppose $u \in \mathbf{U}_{c_{i} \alpha_{i}+c_{j} \alpha_{j}}$. Then $\eta \in M_{s-2 c_{i}, t-2 c_{j}}$, so using Theorem 3.3.1 and the previous case,

$$
T_{i} T_{j}(u \eta)=T_{i} T_{j}(u) T_{i} T_{j}(\eta)=T_{j} T_{i}(u) \pi^{\left(s-2 c_{i}\right)\left(t-2 c_{j}\right)} T_{j} T_{i}(\eta)=\pi^{s t} T_{j} T_{i}(u \eta) .
$$

Now we will drop the assumption $|I|=2$ and consider the general case.

Theorem 5.2.4. Suppose that $i \neq j$ in I such that $m=m_{i, j}<\infty$. Let $M$ be an integrable $\mathbf{U}$-module, $\lambda \in P$, and set $\chi(\lambda)=\left\langle\alpha_{i}^{\vee}, \lambda\right\rangle\left\langle\alpha_{j}^{\vee}, \lambda\right\rangle$ and $\lambda^{\prime}=\ldots s_{i} s_{j} s_{i}(\lambda)=$ $\ldots s_{j} s_{i} s_{j}(\lambda)$, where both products have $m$ factors. Then we have the following equalities, where all products have $m$ factors:

(1) $T_{i} T_{j} T_{i} \ldots=T_{j} T_{i} T_{j} \ldots: \mathbf{U} \rightarrow \mathbf{U}$;

(2) $T_{i}^{-1} T_{j}^{-1} T_{i}^{-1} \ldots=T_{j}^{-1} T_{i}^{-1} T_{j}^{-1} \ldots: \mathbf{U} \rightarrow \mathbf{U}$;

(3) $T_{i} T_{j} T_{i} \ldots=\pi^{\chi(\lambda) p(i) p(j)} T_{j} T_{i} T_{j} \ldots: M_{\lambda} \rightarrow M_{\lambda^{\prime}}$.

(4) $T_{i}^{-1} T_{j}^{-1} T_{i}^{-1} \ldots=\pi^{\chi(\lambda) p(i) p(j)} T_{j}^{-1} T_{i}^{-1} T_{j}^{-1} \ldots: M_{\lambda} \rightarrow M_{\lambda^{\prime}}$.

Proof. This is proved almost identically to [L, Theorem 39.4.13], except for (1) in the case $p(i)=p(j)=1$. In this case, let $u \in \mathbf{U}$ and set $u_{1}=T_{i} T_{j} T_{i} \ldots(u)$ and $u_{2}=T_{j} T_{i} T_{j} \ldots(u)$. Take any integrable $\mathbf{U}$-module $M$, and suppose $m \in M_{\lambda}$. 
Set $\nu=|u|$. Since $i, j \in I_{\overline{1}}$, note that $\left\langle\alpha_{i}^{\vee}, \nu^{\prime}\right\rangle,\left\langle\alpha_{j}^{\vee}, \nu^{\prime}\right\rangle \in 2 \mathbb{Z}$, so in particular $\chi(\lambda+\nu) \equiv \chi(\lambda)$ modulo 2. Then we have

$$
\begin{aligned}
u_{1} T_{j} T_{i} T_{j} \ldots(m) & =\pi^{\chi(\lambda)} u_{1} T_{i} T_{j} T_{i} \ldots(m) \\
& =\pi^{\chi(\lambda)} T_{i} T_{j} T_{i} \ldots(u m) \\
& =\pi^{\chi(\lambda)+\chi(\lambda+\nu)} T_{j} T_{i} T_{j} \ldots(u m) \\
& =u_{2} T_{j} T_{i} T_{j} \ldots(m) .
\end{aligned}
$$

Then $u_{1}-u_{2}$ acts as 0 on any integrable module $M$, and thus $u_{1}=u_{2}$ by CHW1, Proposition 2.7.2].

As a result of Theorem 5.2.4 we see that $\mathbf{U}$ carries an action of the braid group $B$. In particular, for $w \in W$ we may define $T_{w}=T_{i_{1}} \cdots T_{i_{d}}$ if $w=s_{i_{1}} \cdots s_{i_{d}}$ is a reduced expression. As usual, we have $T_{w_{1}} T_{w_{2}}=T_{w_{1} w_{2}}$ if $\ell\left(w_{1} w_{2}\right)=\ell\left(w_{1}\right)+\ell\left(w_{2}\right)$. It follows by Theorem 3.3.1(c) that

$$
T_{w}\left(K_{\mu}\right)=K_{w(\mu)}, \quad T_{w}\left(J_{\mu}\right)=J_{w(\mu)} .
$$

For the integrable $\mathbf{U}$-modules, the situation is slightly more complicated. Let $\lambda \in P$. The spin of the block $\mathcal{O}_{\lambda}$ is a binary sequence $\operatorname{spin}(\lambda) \in\{0,1\}^{I}$ such that

$$
\operatorname{spin}(\lambda)_{i} \equiv \begin{cases}0 & \text { if } i \in I_{\overline{0}}, \quad(\bmod 2) . \\ \left\langle\alpha_{i}^{\vee}, \lambda\right\rangle & \text { if } i \in I_{\overline{1}}\end{cases}
$$

Note that $\operatorname{spin}(\lambda+\nu)=\operatorname{spin}(\lambda)$ for any $\nu \in Q$ by condition (P1) on the GCM $A$. In particular, spin is an invariant of the block $\mathcal{O}_{\lambda}$. We also define the spin-parity function $p_{\lambda}: I \rightarrow\{0,1\}$ via $p_{\lambda}(i)=\operatorname{spin}(\lambda)_{i}$.

Corollary 5.2.5. Let $\lambda \in P$ and $M \in \mathcal{O}_{\lambda}$. Then the spin braid group $B\left(A, p_{\lambda}\right)$ acts on $M$.

5.3. Reduced expressions and admissibility. The braid operators can be used to inductively construct a PBW basis for subspaces of $\mathbf{U}$ using the approach in [L, Chapter 40] almost without modification. For the readers convenience, we will recall the essential results.

Lemma 5.3.1. Assume that $i \neq j \in I$ and let $m=m_{i j} \leq \infty$. Let $p$ be an integer such that $0 \leq p \leq m$. Define the notations

$$
T_{i, j ; p}^{\prime}=\underbrace{\ldots T_{i} T_{j} T_{i}}_{\mathrm{p} \text { factors }}, \quad T_{i, j ; p}^{\prime \prime}=\underbrace{\ldots T_{i}^{-1} T_{j}^{-1} T_{i}^{-1}}_{\mathrm{p} \text { factors }} .
$$

and let $\mathbf{U}^{+}(i, j)$ be the $\mathbf{U}_{J}^{0}$-subalgebra of $\mathbf{U}$ generated by $E_{i}, E_{j}$. Then $T_{i, j ; p}^{\prime}\left(E_{j}\right)$, $T_{i, j ; p}^{\prime \prime}\left(E_{j}\right) \in \mathbf{U}^{+}(i, j)$.

Proof. If $m<\infty$, then the statement follows from the explicit calculations in the proof of Lemma 5.1.1. In the case $m=\infty$, the proof is virtually identical to that of [L Lemma 40.1.1], and we omit the details.

Lemma 5.3.2. Let $w=s_{i_{1}} \ldots s_{i_{n}}$ be a reduced expression in $W$. Then we have that $T_{i_{1}} \ldots T_{i_{n-1}}\left(E_{i_{n}}\right)$ and $T_{i_{1}}^{-1} \ldots T_{i_{n-1}}^{-1}\left(E_{i_{n}}\right)$ are in $\mathbf{U}_{J}^{+}$.

Proof. This is proved exactly as [L, Lemmas 40.1.2, 40.1.3] using Lemma 5.3.1. 
Proposition 5.3.3. Let $w \in W$ and $\mathbf{h}=\left(i_{1}, \ldots, i_{n}\right)$ be a sequence in $I$ such that $w=s_{i_{1}} \ldots s_{i_{n}}$ is a reduced expression. Then the following statements hold.

(1) The sequence $\mathbf{h}$ is admissible.

(2) The elements $E_{i_{1}}^{\left(c_{1}\right)} T_{i_{1}}\left(E_{i_{2}}^{\left(c_{2}\right)}\right) \ldots T_{i_{1}} T_{i_{2}} \ldots T_{i_{n-1}}\left(E_{i_{n}}^{\left(c_{n}\right)}\right)$ with $c_{1}, \ldots, c_{n} \in \mathbb{N}$ form a $\mathbf{U}_{J}^{0}$-basis of a subspace $\mathbf{U}_{J}^{+}(w)$ of $\mathbf{U}_{J}^{+}$, and this subspace does not depend on the sequence $\mathbf{h}$.

(3) The elements $E_{i_{1}}^{\left(c_{1}\right)} T_{i_{1}}^{-1}\left(E_{i_{2}}^{\left(c_{2}\right)}\right) \ldots T_{i_{1}}^{-1} T_{i_{2}}^{-1} \ldots T_{i_{n-1}}^{-1}\left(E_{i_{n}}^{\left(c_{n}\right)}\right)$ form a $\mathbf{U}_{J}^{0}$-basis of the subspace $\mathbf{U}_{J}^{+}(w)$.

(4) If $i \in I$ such that $l\left(s_{i} w\right)<l(w)$, then $E_{i} \mathbf{U}_{J}^{+}(w) \subset \mathbf{U}_{J}^{+}(w)$.

Proof. The proof of (1), (4), and the independence of $\mathbf{U}_{J}^{+}(w)$ from the choice of $\mathbf{h}$ is proved exactly as in [L, Lemma 40.2.1]. The linear independence of the elements in (2) and (3) is proved exactly as in the rank 2 case. To wit, by part (1) and Proposition 4.3.4 these elements are pairwise orthogonal and if $x=$ $E_{i_{1}}^{\left(c_{1}\right)} T_{i_{1}}\left(E_{i_{2}}^{\left(c_{2}\right)}\right) \ldots T_{i_{1}} T_{i_{2}} \ldots T_{i_{n-1}}\left(E_{i_{n}}^{\left(c_{n}\right)}\right)$ then $(x, x)$ is not a zero divisor, and thus the elements are linearly independent.

In particular, we obtain a basis when the Cartan datum is of finite type as follows.

Corollary 5.3.4. Suppose the Cartan datum is of finite type and $w_{0}=s_{i_{1}} \ldots s_{i_{n}}$ is a reduced expression for the longest element of $W$. Then the elements

$$
\left\{E_{i_{1}}^{\left(c_{1}\right)} T_{i_{1}}\left(E_{i_{2}}^{\left(c_{2}\right)}\right) \ldots T_{i_{1}} T_{i_{2}} \ldots T_{i_{n-1}}\left(E_{i_{n}}^{\left(c_{n}\right)}\right) \mid c_{1}, \ldots, c_{n} \in \mathbb{N}\right\}
$$

form a $\mathbf{U}_{J}^{0}$-basis of $\mathbf{U}_{J}^{+}$. Likewise, the elements

$$
\left\{E_{i_{1}}^{\left(c_{1}\right)} T_{i_{1}}^{-1}\left(E_{i_{2}}^{\left(c_{2}\right)}\right) \ldots T_{i_{1}}^{-1} T_{i_{2}}^{-1} \ldots T_{i_{n-1}}^{-1}\left(E_{i_{n}}^{\left(c_{n}\right)}\right) \mid c_{1}, \ldots, c_{n} \in \mathbb{N}\right\}
$$

for various $c_{1}, \ldots, c_{n} \in \mathbb{N}$ form a $\mathbf{U}_{J}^{0}$-basis of $\mathbf{U}_{J}^{+}$.

\section{REFERENCES}

[B] J. Beck, Convex bases of PBW type for quantum affine algebras, Comm. Math. Phys. 165 (1994), 193-199.

[BKM] G. Benkart, S.-J. Kang and D. Melville, Quantized enveloping algebras for Borcherds superalgebras, Trans. AMS. 350 (1998), 3297-3319.

[CK] S. Cautis and J. Kamnitzer, Braiding via geometric Lie algebra actions, Comp. Math. 148 (2012), 464-506.

[CKL] S. Cautis, J. Kamnitzer, and A. Licata, Derived equivalences for cotangent bundles of Grassmannians via categorical $\mathfrak{s l}_{2}$ actions, J. Reine Angew. Math. 675 (2013), 53-99.

$[\mathrm{CR}]$ J. Chuang and R. Rouquier, Derived equivalences for symmetric groups and $\mathfrak{s l}_{2}$ categorification, Ann. of Math. (2) 167 (2008), no. 1, 245-298.

[C] S. Clark, Quantum supergroups IV. The modified form, 278 (2014), 493-528.

[CW] S. Clark and W. Wang, Canonical basis for quantum osp(1|2), Lett. Math. Phys. 103 (2013), 207-231.

[CFLW] S. Clark, Z. Fan, Y. Li and W. Wang, Quantum supergroups III. Twistors, Comm. Math. Phys. 332 (2014), 415-436.

[CHW1] S. Clark, D. Hill and W. Wang, Quantum supergroups I. Foundations, Transform. Groups 18 (2013), 1019-1053.

[CHW2] S. Clark, D. Hill and W. Wang, Quantum supergroups II. Canonical basis, Represent. Theory 18 (2014), pp. 278-309.

[CHW3] S. Clark, D. Hill and W. Wang, Quantum shuffles and quantum supergroups of basic type, Quantum Topology (to appear), arXiv:1310.7523 
[HW] D. Hill and W. Wang, Categorification of Quantum Kac-Moody Superalgebras, Trans. AMS 367 (2015), 1183-1216.

[L2] G. Lusztig, Quantum deformations of certain simple modules over enveloping algebras, Adv. Math. 70 (1988), 237-249.

[L3] G. Lusztig, Quantum groups at roots of 1, Geom. Dedicata. 35 (1990), 89-114.

[L] G. Lusztig, Introduction to quantum groups, volume 110 of Progress in Mathematics. Birkhäuser Boston Inc., Boston, MA, 1993.

[Sai] Y. Saito, $P B W$ basis of quantized universal enveloping algebras, Publ. Res. Inst. Math. Sci. 30 (1994), 209-232.

Max Plank Institute for Mathematics, 53119 Bonn, Germany.

E-mail address: se.clark@mpim-bonn.mpg.de

Department of Mathematics and Statistics, Washington State University - VancouVER, VANCOUVER, WA 98686, USA.

E-mail address: david.hill@wsu.edu 